DOI 10.4171/JEMS/678

Sebastian Casalaina-Martin · Samuel Grushevsky

Klaus Hulek · Radu Laza

\title{
Extending the Prym map to toroidal compactifications of the moduli space of abelian varieties (with an appendix by Mathieu Dutour Sikirić)
}

Received May 1, 2014 and in revised form December 15, 2014

\begin{abstract}
The main purpose of this paper is to present a conceptual approach to understanding the extension of the Prym map from the space of admissible double covers of stable curves to different toroidal compactifications of the moduli space of principally polarized abelian varieties. By separating the combinatorial problems from the geometric aspects we can reduce this to the computation of certain monodromy cones. In this way we not only shed new light on the extension results of Alexeev, Birkenhake, Hulek, and Vologodsky for the second Voronoi toroidal compactification, but we also apply this to other toroidal compactifications, in particular the perfect cone compactification, for which we obtain a combinatorial characterization of the indeterminacy locus, as well as a geometric description up to codimension six, and an explicit toroidal resolution of the Prym map up to codimension four.
\end{abstract}

Keywords. Moduli, Prym varieties, period maps, abelian varieties

\section{Introduction}

A fundamental tool in the study of algebraic curves is the theory of Jacobians. Assigning to a curve its principally polarized Jacobian defines the Torelli period map $M_{g} \rightarrow A_{g}$ from the coarse moduli space of curves of genus $g$ to the coarse moduli space of principally polarized abelian varieties (ppav) of dimension $g$. It is a well known fact, due to Mumford and Namikawa [Nam80], that the Torelli map extends to a morphism $\bar{M}_{g} \rightarrow \bar{A}_{g}^{V}$ from the Deligne-Mumford compactification to the second Voronoi toroidal

S. Casalaina-Martin: Department of Mathematics, University of Colorado, Boulder, CO 80309, USA; e-mail: casa@ math.colorado.edu

S. Grushevsky: Department of Mathematics, Stony Brook University, Stony Brook, NY 11794, USA; e-mail: sam@math.sunysb.edu

K. Hulek: Institut für Algebraische Geometrie, Leibniz Universität Hannover, 30060 Hannover, Germany; e-mail: hulek@ math.uni-hannover.de

R. Laza: Department of Mathematics, Stony Brook University, Stony Brook, NY 11794, USA; e-mail: rlaza@math.sunysb.edu

M. Dutour Sikirić: Rudjer Bosković Institute, Bijenicka 54, 10000 Zagreb, Croatia; e-mail:mathieu.dutour@gmail.com

Mathematics Subject Classification (2010): Primary 14H40, 14K10, 14H10 
compactification. More recently, Alexeev and Brunyate [AB12] have studied extensions of the Torelli map to other toroidal compactifications and have shown that the period map extends to a morphism to the perfect cone compactification $\bar{A}_{g}^{P}$, but not to a morphism to the central cone compactification $\bar{A}_{g}^{C}$ for $g \geq 9$, disproving a conjecture of Namikawa.

While the Torelli map is injective for all $g$, for $g \geq 4$ it is not dominant. One geometric approach to understanding higher-dimensional ppavs is via Prym varieties, which are ppavs associated to connected étale double covers of curves. Associating to a cover its principally polarized Prym variety defines the Prym period map $R_{g+1} \rightarrow A_{g}$, where $R_{g+1}$ is the coarse moduli space of connected étale double covers of curves of genus $g+1$. The Prym period map is dominant for $g \leq 5$, and has been used to provide a geometric approach to the Schottky problem for $g=4,5$, to study the rationality of threefolds, and to give a better understanding of the geometry of $A_{4}$ and $A_{5}$.

In contrast to the case of Jacobians, it has been known since the work of Friedman and Smith [FS86] that the Prym period map does not extend to a morphism from Beauville's moduli space $\bar{R}_{g+1}$ of admissible double covers to any of the standard toroidal compactifications. Subsequent work of Alexeev, Birkenhake, and Hulek [ABH02] and Vologodsky [Vol02] identifies the indeterminacy locus of the rational map $\bar{R}_{g+1} \rightarrow \bar{A}_{g}^{V}$; it is the closure of the locus of so-called Friedman-Smith covers with at least four nodes (see §6). In this paper, we investigate the problem of extending the Prym map to other toroidal compactifications. Our main results are:

- A complete combinatorial characterization of the indeterminacy locus of the Prym map to the perfect and central cone compactifications (Theorem 5.6). The techniques also give a complete combinatorial characterization of the indeterminacy locus of the Prym map to the second Voronoi compactification, providing another proof of [ABH02, Thm. 3.2].

- A geometric characterization of the indeterminacy locus of the Prym map $\bar{R}_{g+1} \rightarrow \bar{A}_{g}^{P}$ to the perfect cone compactification up to codimension 6 in $\bar{R}_{g+1}$ in terms of Friedman-Smith covers (Theorem 7.1).

- An explicit resolution of the Prym map $\bar{R}_{g+1} \rightarrow-\bar{A}_{g}^{P}$ up to codimension 4 (Theorem 8.1). This also resolves the Prym map to $\bar{A}_{g}^{V}$ and $\bar{A}_{g}^{C}$ up to codimension 4.

In Appendix E, Mathieu Dutour Sikirić also proves an extension result for the Prym map to the central cone compactification (Theorem E.1).

In this paper, we approach the extension problem for the Prym map in terms of the Hodge-theoretic framework of a general period map $\mathcal{M} \rightarrow \mathcal{D} / \Gamma$ from a moduli space to a classical period domain. This allows us to determine the conditions for extensions of period maps to moduli spaces that are compactified so that the monodromy transformations are of Picard-Lefschetz type (i.e. given by rank 1 forms). In this way we separate the geometric aspects of the problem from the combinatorial issues involved in dealing with various admissible cone decompositions.

In particular, the approach unifies the arguments for Jacobians and Pryms, and we discuss the Torelli map throughout for motivation. As a result, we also get a new proof of the extension results of [ABH02] for $\bar{R}_{g+1} \rightarrow \bar{A}_{g}^{V}$. In [ABH02], the authors have the additional goal of determining compactified Pryms as stable semiabelic pairs; focusing 
here on the extension condition allows us to give a more direct, Hodge-theoretic argument. With the work in [ABH02], translating from our results to the language of stable semiabelic pairs is straightforward (\$2.4, §9). In addition, one of our original motivations for this work was investigating the extension of the period map for cubic threefolds to a morphism from a suitable GIT compactification of the moduli space of threefolds to a suitable compactification of $A_{5}$, stemming from our work [CML09] and [CML13], and using some of the results of our work [GH12]. The methods we use in this paper apply in that setting also, and we will return to the study of the period map for cubic threefolds in subsequent work.

A few words about the structure of the paper. We start in Section 1 by reviewing some basic facts about the toroidal compactifications (second Voronoi, perfect, central) that we consider in our paper. We then discuss (Section 2) the general framework of degenerations of Hodge structures and the connection to toroidal compactifications. This is mostly standard (see e.g. [Cat84] for an exposition), but we find it convenient to include a short discussion of this, adapted to our needs. In Section 3, we briefly review the standard compactification of the moduli of Prym varieties by admissible covers [Bea77] and the associated combinatorial data (graphs with an involution, etc.). In Section 4, we specialize the discussion of Section 2 to curves and Prym varieties and discuss the computation of the monodromy cones in terms of the dual graph. The monodromy cone for Jacobians is classical (e.g. [Nam76]) and that of Pryms is essentially contained in [FS86] and [ABH02]. Nonetheless, we believe that our presentation unifies, simplifies, and clarifies some of the arguments in the literature. Our goal will be to apply similar techniques to the study of other moduli spaces via Hodge theory in the future.

With these preliminaries, new results start in Section 5, where we recast the extension criteria for the Torelli map, and then prove combinatorial criteria, in terms of the dual graph, for the extension of the Prym map to various toroidal compactifications of $A_{g}$, obtaining Theorem 5.6 and thus giving in addition a new proof of [ABH02, Thm. 3.2]. We then proceed to relate these combinatorial conditions to geometric conditions on admissible covers. The so-called Friedman-Smith covers are central to this discussion and we describe in Section 6 their monodromy in detail: in Subsection 6.2 we compute the monodromy cones, and in Theorem 6.4 we discuss their properties with respect to the fans defining different toroidal compactifications. In Section 7, we use these computations to describe the indeterminacy locus of the Prym map geometrically, and it is interesting to note that this behavior for the perfect cone compactification is quite different from that for the second Voronoi compactification. We are able to give a complete geometric characterization of the indeterminacy locus of the Prym map to the perfect cone compactification $\bar{A}_{g}^{P}$ up to codimension 6 (Theorem 7.1), utilizing the recent results of Melo and Viviani [MV12].

The computations also allow us to describe the resolution of the period map in terms of explicit, toroidal modifications of the moduli space of admissible covers. In Section 8 we describe the resolution of the period map to the perfect cone compactification completely up to codimension 4 (Theorem 8.1). In Section 9 we start a discussion on the fibers of the Prym map. More precisely, we discuss which types of admissible covers are mapped to which strata. This also provides another link to [ABH02] since we discuss the relationship 
between the monodromy cones and the degeneration data of 1-parameter families, which in turn determine semiabelic varieties which are limits of Pryms.

Many of the arguments in the paper regarding the Prym map in low codimension rely on working through a number of examples, and explicit computations of monodromy cones. These are somewhat lengthy and technical, and to maintain the structural unity of the argument we collect these explicit computations in the appendices. Appendix A treats the combinatorics of the Friedman-Smith cones and relates these to various cone decompositions. In Appendix B we discuss some examples where the Prym map extends; this comes down to proving that certain monodromy cones belong to either the second Voronoi, perfect cone or central cone decomposition. Appendix $\mathrm{C}$ contains some lengthy calculations where we discuss further degenerations of Friedman-Smith examples. In particular we compute their monodromy cones and discuss to which, if any, cone decompositions these belong. Finally, in Appendix D we discuss a method which allows us to simplify certain monodromy cones and thus to reduce to previous calculations.

\section{Notation}

We will use calligraphic letters to refer to moduli stacks (e.g. $\mathcal{A}_{g}, \mathcal{R}_{g+1}$, etc.), and italic letters for the associated coarse moduli spaces (e.g. $A_{g}, R_{g+1}$, etc.). Since all the spaces occurring here (with the exception of Alexeev's stack of stable semiabelic pairs) are Deligne-Mumford stacks, all the period maps are assumed to be locally liftable, and the extensions are insensitive to finite covers, there is essentially no difference between using stacks or the associated coarse moduli space. In fact, we will typically stick to the coarse moduli space, except for the situations where we want to emphasize the modular meaning.

\section{Brief review of toroidal compactifications}

In this section, we briefly review the theory of toroidal compactifications of $A_{g}$ (see $\left[\mathrm{AM}^{+} 10\right]$, [Nam80] and [FC90] for more details), focusing on the three classically known toroidal compactifications (up to refinement of fans, i.e. blow-ups), that is the perfect cone (also known as first Voronoi), second Voronoi, and central cone compactification. Primarily the purpose here is to fix the notation and terminology needed later.

Notation 1.1. As is customary, when necessary, we will use subscripts (e.g. $H_{\mathbb{Z}}$ ) to indicate the coefficients for modules and algebraic groups. Unless specified, the coefficients are either $\mathbb{Q}$ or $\mathbb{R}$.

\subsection{The Satake-Baily-Borel compactification}

Fix a free abelian group $H$ of rank $2 g$, and a non-degenerate, skew-symmetric, bilinear form $Q$ on $H$. We let $D$ be the classifying space of polarized weight 1 Hodge structures on $H$ :

$$
D:=\left\{F \in \operatorname{Grass}\left(g, H_{\mathbb{C}}\right): Q(F, F)=0, i Q(F, \bar{F})>0\right\} \cong G_{\mathbb{R}} / K
$$


where $G_{\mathbb{R}} \cong \operatorname{Sp}(2 g, \mathbb{R})$ and $K=U(r)$ is the maximal compact subgroup. By taking $Q$ to be the standard symplectic form, $D$ can be (canonically) identified with the Siegel upper half-space $\mathfrak{H}_{g}$, the space of symmetric $g \times g$ complex matrices with positive definite imaginary part. The fractional linear transformations give an action of $G_{\mathbb{Z}}=\operatorname{Sp}(2 g, \mathbb{Z})$ on $D \cong \mathfrak{H}_{g}$, and we set

$$
A_{g}:=\mathfrak{H}_{g} / \operatorname{Sp}(2 g, \mathbb{Z}) .
$$

The Satake-Baily-Borel (SBB) compactification $A_{g}^{*}$ is a normal, projective compactification of $A_{g}$ that admits a stratification

$$
A_{g}^{*}=A_{g} \sqcup A_{g-1} \sqcup \cdots \sqcup A_{0} .
$$

We recall that $A_{g}^{*}$ and the above stratification are obtained (set-theoretically) by adding to $D$ the so-called rational boundary components $F_{W_{0}}$, and then taking the quotient with respect to the natural $G_{\mathbb{Z}}=\operatorname{Sp}(2 g, \mathbb{Z})$ action. Namely, the rational boundary components $F_{W_{0}}$ of $D$ correspond to the choice of rational maximal parabolic subgroups $P_{W_{0}} \subset \operatorname{Sp}\left(Q, H_{\mathbb{Q}}\right)$, which in turn correspond to the choice of a totally isotropic subspace $W_{0} \subseteq H_{\mathbb{Q}}$ (of which $P_{W_{0}}$ is then the stabilizer). Note that since $\operatorname{Sp}(2 g, \mathbb{Z})$ acts transitively on the set of isotropic subspaces $W_{0}$ of $H_{\mathbb{Q}}$ of fixed dimension, the set of rational boundary components is essentially indexed by $v\left(=\operatorname{dim} W_{0}\right) \in\{0, \ldots, g\}$. Furthermore, the choice of $W_{0}$ defines a weight filtration on $H_{\mathbb{Q}}$ :

$$
W_{-1}:=\{0\} \subseteq W_{0} \subseteq W_{1}:=\left(W_{0}\right)_{Q}^{\perp} \subseteq W_{2}:=H_{\mathbb{Q}} .
$$

The polarization $Q$ induces a polarization (non-degenerate symplectic form) $\bar{Q}$ on $\mathrm{Gr}_{1}^{W}=$ $W_{1} / W_{0}$. It is then standard (e.g. [Cat84, p. 84]) that the boundary component $F_{W_{0}}$ is the classifying space $D_{g^{\prime}}$ (with $g^{\prime}=g-v$ ) of $\bar{Q}$-polarized Hodge structures on $\mathrm{Gr}_{1}^{W}$, giving the component $A_{g^{\prime}}=F_{W_{0}} / G_{\mathbb{Z}}$ of $A_{g}^{*}$ (N.B. $F_{\{0\}}=D$, and after the identification $F_{W_{0}}=D_{g^{\prime}}=\mathfrak{H}_{g^{\prime}}$, the action of $G_{\mathbb{Z}}$ restricts to the action of $\operatorname{Sp}\left(2 g^{\prime}, \mathbb{Z}\right)$.)

\subsection{Toroidal compactifications}

Toroidal compactifications are certain refinements of the SBB compactification $A_{g}^{*}$, depending on a choice of a compatible collection of admissible cone decompositions, $\Sigma$. Each such choice gives a compactification $\bar{A}_{g}^{\Sigma}$ with a canonical map $\bar{A}_{g}^{\Sigma} \rightarrow A_{g}^{*}$. Here we review a few points about the construction from the perspective of Hodge theory (essentially following [Cat84]).

The construction is relative over $A_{g}^{*}$, and one starts by considering a totally isotropic subspace $W_{0} \subseteq H_{\mathbb{Q}}$ of dimension $v \leq g$ and the corresponding boundary component of $A_{g}^{*}$. Consider then the real Lie subalgebra of $\mathfrak{s p}\left(Q, H_{\mathbb{R}}\right)$ preserving $W_{0}$ :

$$
\mathfrak{n}\left(W_{0}\right):=\left\{N \in \mathfrak{s p}\left(Q, H_{\mathbb{R}}\right): \operatorname{Im}(N) \subseteq W_{0}\right\} .
$$

Then for any $N \in \mathfrak{n}\left(W_{0}\right)$ we have $N^{2}=0$, and thus $N$ defines a weight filtration compatible with that induced by $W_{0}$ (see (1.1)). In other words, we have

$$
\operatorname{Im}(N)=W_{0}(N) \subseteq W_{0} \subseteq W_{1}=W_{0}^{\perp} \subseteq W_{1}(N)=\operatorname{ker}(N)=\operatorname{Im}(N)^{\perp},
$$


and in particular a natural surjection

$$
\operatorname{Gr}_{2}^{W}\left(:=W_{2} / W_{1}\right) \rightarrow \operatorname{Gr}_{2}(N)\left(:=W_{2}(N) / W_{1}(N)\right) .
$$

Furthermore, since $N$ is a nilpotent symplectic endomorphism, we get a natural isomorphism

$$
\begin{aligned}
\mathrm{Gr}_{2}(N) & \stackrel{N}{\rightarrow} \mathrm{Gr}_{0}(N) \stackrel{Q(N(\cdot), \cdot)}{\longrightarrow} \mathrm{Gr}_{2}(N)^{\vee}, \\
v & \mapsto N(v) \mapsto Q(N(\cdot), v),
\end{aligned}
$$

which can be interpreted as giving a non-degenerate bilinear form $Q_{N}$ on $\operatorname{Gr}_{2}(N)$. The form $Q_{N}$ turns out to be symmetric, and by pull-back can be viewed as a form on $\mathrm{Gr}_{2}^{W}$; thus there is a natural map (defined over $\mathbb{Q}$ )

$$
\mathfrak{n}\left(W_{0}\right) \stackrel{\sim}{\rightarrow} \operatorname{Hom}\left(\operatorname{Sym}^{2} \mathrm{Gr}_{2}^{W}, \mathbb{R}\right),
$$

which (as is not hard to see) is an isomorphism.

As described above, $\mathfrak{n}\left(W_{0}\right)$ is canonically identified with the Lie algebra of symmetric bilinear forms (or equivalently symmetric $g^{\prime} \times g^{\prime}$ matrices with $g^{\prime}=g-v$ ) on $\mathrm{Gr}_{2}^{W}$. With this identification, we consider the cone of positive definite $g^{\prime} \times g^{\prime}$ symmetric matrices

$$
\mathfrak{n}\left(W_{0}\right)^{+}:=\left\{N \in \mathfrak{n}\left(W_{0}\right): Q_{N} \text { is positive definite }\right\} .
$$

Let $\Sigma$ be a compatible collection of admissible cone decompositions (see $\S 1.3$ ). Now for each cone $\sigma_{W_{0}} \in \Sigma_{W_{0}}$, there is an associated space $B\left(\sigma_{W_{0}}\right)$ together with a map $B\left(\sigma_{W_{0}}\right) \rightarrow F_{W_{0}}$, where $F_{W_{0}}$ is the rational boundary component associated to $W_{0}$ (see e.g. [Cat84, p. 91]). These maps are compatible in the sense that if $\tau_{W_{0}} \leq \sigma_{W_{0}}$ is a face, then there is a commutative diagram

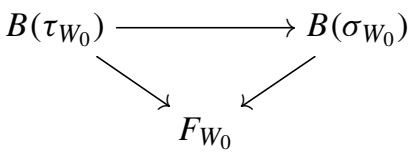

One then sets $D^{\Sigma}=\bigcup_{W_{0}} \bigcup_{\sigma_{W_{0}} \in \Sigma_{W_{0}}} B\left(\sigma_{W_{0}}\right)$. The action of $G_{\mathbb{Z}}=\operatorname{Sp}(2 g, \mathbb{Z})$ extends to an action on $D^{\Sigma}$, and then (set-theoretically) $\bar{A}_{g}^{\Sigma}=D^{\Sigma} / G_{\mathbb{Z}}$, inducing also a natural map $\bar{A}_{g}^{\Sigma} \rightarrow A_{g}^{*}$.

\subsection{Admissible cone decompositions for quadratic forms}

We now review some basic terminology and results about cone decompositions. Let $\Lambda$ be a free $\mathbb{Z}$-module of rank $g$. The space of quadratic forms on $\Lambda$ is $\left(\operatorname{Sym}^{2} \Lambda\right)^{\vee}$, which comes equipped with a natural diagonal action of $\operatorname{GL}(\Lambda)=\operatorname{Aut}_{\mathbb{Z}}(\Lambda)$. One considers the open cone

$$
C \subset\left(\operatorname{Sym}^{2} \Lambda\right)^{\vee} \otimes_{\mathbb{Z}} \mathbb{R},
$$

of positive definite quadratic forms, and then lets $\bar{C}^{\mathbb{Q}}$ be its rational closure. Obviously, $C$ and $\bar{C}^{\mathbb{Q}}$ are $\operatorname{GL}(\Lambda)$-invariant. For any subgroup $\Gamma \subseteq \operatorname{GL}(\Lambda)$ (typically we will be 
interested in $\Gamma=\mathrm{GL}(\Lambda)$ ), a $\Gamma$-admissible rational polyhedral decomposition $\Sigma$ (for short, an admissible decomposition) of $C$ is a $\Gamma$-invariant collection of (rational, convex, polyhedral) subcones covering $\bar{C}^{\mathbb{Q}}$ which satisfies certain natural axioms (see [Nam80] or [FC90, Ch. IV, Def. 2.2, p. 96] for details), most notably the requirement that there are only finitely many orbits of cones of $\Sigma$ modulo the action of $\Gamma$.

For the construction of the toroidal compactifications $\bar{A}_{g}^{\Sigma}$ one requires an admissible decomposition for the space of quadratic forms associated to each isotropic subspace $W_{0}$ (see (1.4)). As discussed, all isotropic subspaces $W_{0}$ of fixed dimension are conjugate, and thus what one needs is an admissible decomposition for each lattice $\Lambda^{\prime}$ of rank $0 \leq$ $g^{\prime} \leq g$, compatible in the following sense. We say that $\Sigma^{\prime}$ and $\Sigma$ are compatible if there exists a surjection $\Lambda \rightarrow \Lambda^{\prime}$ such that $\Sigma^{\prime}$ is obtained from $\Sigma$ via pull-back by the natural inclusion $\bar{C}^{\mathbb{Q}}\left(\Lambda^{\prime}\right) \subseteq \bar{C}^{\mathbb{Q}}(\Lambda)$. If this is the case for one surjection $\Lambda \rightarrow \Lambda^{\prime}$, it will be true for all surjections. In particular, specifying an admissible decomposition for $\Lambda$ then uniquely specifies compatible admissible decompositions for all lattices $\Lambda^{\prime}$ of smaller rank. In short, all we need to define a toroidal compactification $\bar{A}_{g}^{\Sigma}$ is an admissible cone decomposition for the rank $g$ lattice.

Three admissible decompositions are classically known for $A_{g}$, namely the so-called second Voronoi decomposition, the perfect cone (or first Voronoi) decomposition, and the central cone decomposition (these can, of course, be further subdivided). These decompositions are discussed in [Nam80, §8, §9]. We shall address all three decompositions and the associated toroidal compactifications. Though we will not review their definitions (the interested reader should see [Nam80]), we will discuss the relevant facts about them in the following subsection. There is also another admissible decomposition, namely that into $C$-types [RB78], which is less known to algebraic geometers. This coincides with the second Voronoi decomposition for $g \leq 4$, but for $g \geq 5$ second Voronoi is a proper refinement of the $C$-type decomposition. To our knowledge no geometric interpretation of the corresponding toroidal compactification is known.

Finally, we recall some terminology. A cone $\sigma \subseteq \bar{C}^{\mathbb{Q}}$ is called basic if the integral generators of its 1-dimensional faces can be completed to a $\mathbb{Z}$-basis of $\left(\operatorname{Sym}^{2} \Lambda\right)^{\vee}$. It is called simplicial if these generators can be completed to a $\mathbb{Q}$-basis, i.e. if they are linearly independent.

\subsection{Admissible cone decompositions and rank 1 quadrics}

In the geometric context of our paper, we will only be interested in cones spanned by rank 1 quadrics (i.e. squares of linear forms), since our (log of) monodromy operators will be rank 1 . For such cones it is essentially a combinatorial problem to decide if they belong to the second Voronoi, perfect, or central cone decompositions. These results are well known and we will refer the reader to [AB12] and [MV12] for further details.

For $\ell_{1}, \ldots, \ell_{n} \in \Lambda_{\mathbb{R}}^{\vee} \backslash\{0\}$, let $\sigma:=\mathbb{R}_{\geq 0}\left\langle\ell_{i}^{2}\right\rangle_{i=1}^{n}$ be the corresponding cone generated by rank 1 quadrics in $\operatorname{Sym}^{2} \Lambda_{\mathbb{R}}^{\vee}$. Given a basis for $\Lambda$, we will often refer to the cone $\sigma$ by writing the matrix whose $i$-th row is the expression for $\ell_{i}$ in terms of the dual basis to the given basis, and to any such matrix we will associate such a cone. 
In this set-up, we then have the following combinatorial results that determine whether a set of linear forms in $\Lambda^{\vee}$ generate a cone contained in a cone of one of the three standard admissible decompositions.

Lemma 1.2 (Second Voronoi). Let $\Lambda$ be a free $\mathbb{Z}$-module of rank $g$. Suppose $\ell_{1}, \ldots, \ell_{n}$ $\in \Lambda^{\vee}$ are primitive non-zero linear forms. The following are equivalent:

(1) $\left\{\ell_{1}^{2}, \ldots, \ell_{n}^{2}\right\}$ lie in a common cone of the second Voronoi decomposition.

(2) $\mathbb{R}_{\geq 0}\left\langle\ell_{1}^{2}, \ldots, \ell_{n}^{2}\right\rangle$ is a cone in the second Voronoi decomposition.

(3) Any $\mathbb{R}$-linearly independent subset $\left\{\ell_{j}\right\}_{j \in J} \subseteq\left\{\ell_{1}, \ldots, \ell_{n}\right\}$ is a $\mathbb{Z}$-basis of the $\mathbb{Z}$-module $\mathbb{R}\left\langle\ell_{j}\right\rangle_{j \in J} \cap \Lambda^{\vee}$.

(4) Any $\mathbb{R}$-linearly independent subset $\left\{\ell_{j}\right\}_{j \in J} \subseteq\left\{\ell_{1}, \ldots, \ell_{n}\right\}$ of maximal rank is a $\mathbb{Z}$-basis of the $\mathbb{Z}$-module $\mathbb{R}\left\langle\ell_{j}\right\rangle_{j \in J} \cap \Lambda^{\vee}$.

Proof. This is well known. We direct the reader to [AB12, Lem. 4.5] and the references therein.

One may take as a definition that a matroidal cone is a second Voronoi cone generated by rank 1 quadrics (this is essentially the content of Lemma 1.2). It follows from the lemma that a face of a matroidal cone is matroidal, and that matroidal cones are simplicial. We denote by $\Sigma_{\text {mat }} \subseteq \Sigma_{V}$ the collection of matroidal cones.

To connect the discussion with that of [ABH02], we recall the notion of a dicing. Fix a collection of codimension 1 affine spaces $\left\{H_{i}\right\}_{i \in I}$ in $\Lambda_{\mathbb{R}}$. Let $\mathscr{H}=\bigcup_{i \in I} H_{i}$ be the associated arrangement of affine spaces. The arrangement $\mathscr{H}$ is stratified by the intersections of the $H_{i}$. We say that $\mathscr{H}$ defines a dicing of $\Lambda$ if the union of the 0 -dimensional strata of $\mathscr{H}$ is exactly the lattice $\Lambda$.

Lemma 1.3. Let $\Lambda$ be a free $\mathbb{Z}$-module of rank $g$. Suppose that $\ell_{1}, \ldots, \ell_{n} \in \Lambda^{\vee}$ are $\mathbb{R}$-linearly independent. Then $\ell_{1}, \ldots, \ell_{n}$ form a $\mathbb{Z}$-basis for $\Lambda^{\vee}$ if and only if they determine a dicing of $\Lambda_{\mathbb{R}}$. More precisely, this means that the collection of hyperplanes

$$
H_{i, m}:=\left\{x \in \Lambda_{\mathbb{R}}: \ell_{i}(x)=m\right\}
$$

with $i=1, \ldots, n$ and $m \in \mathbb{Z}$ defines a dicing of $\Lambda$.

Proof. This follows from the definitions and is left to the reader.

Remark 1.4. Associated to a quadratic form $q \in C$ is the so-called Delaunay decomposition of $\Lambda \otimes_{\mathbb{Z}} \mathbb{R}$. The second Voronoi decomposition is defined so that the Delaunay decomposition of a quadric remains unchanged for all quadrics in a given (open) second Voronoi cone. We will only be interested in quadratic forms that lie in second Voronoi cones generated by rank 1 quadrics. In this case, the Delaunay decomposition has a well known and simple description (see [ER94, Thm. 3.2] or [ABH02, proof of Lem. 3.1]): If $\ell_{1}, \ldots, \ell_{n} \in \Lambda^{\vee}$ span $\Lambda_{\mathbb{R}}^{\vee}$, and $\sigma=\mathbb{R}_{\geq 0}\left\langle\ell_{1}^{2}, \ldots, \ell_{n}^{2}\right\rangle$ is a second Voronoi cone, then the Delaunay decomposition for any (positive definite) quadric $q \in \sigma^{\circ}$ is given by the (dicing) hyperplane arrangement associated to $\ell_{1}, \ldots, \ell_{n}$. 
Lemma 1.5 (Perfect cone). Let $\Lambda$ be a free $\mathbb{Z}$-module of rank $g$. Suppose $\ell_{1}, \ldots, \ell_{n}$ $\in \Lambda^{\vee}$ are primitive non-zero linear forms. The following are equivalent:

(1) $\left\{\ell_{1}^{2}, \ldots, \ell_{n}^{2}\right\}$ lie in the same cone of the perfect cone decomposition.

(2) There exists a quadratic form $Q$ on $\Lambda_{\mathbb{R}}^{\vee}$ such that

(a) $Q(\ell)>0$ for all $\ell \in \Lambda_{\mathbb{R}}^{\vee} \backslash\{0\}$, i.e. $Q$ is positive definite.

(b) $Q(\ell) \geq 1$ for all $\ell \in \Lambda^{\vee} \backslash\{0\}$.

(c) $Q\left(\ell_{i}\right)=1, i=1, \ldots, n$.

Proof. This follows from the definition of the perfect cone decomposition in [Nam80]. (See also the proof of [AB12, Thm. 4.7].)

Remark 1.6. Since cones in the perfect cone decomposition are generated by rank 1 quadrics, a cone in the perfect cone decomposition is a second Voronoi cone if and only if it is matroidal (i.e. $\Sigma_{P} \cap \Sigma_{V} \subseteq \Sigma_{\text {mat }}$ ). Recently Melo and Viviani [MV12, Thm. A] showed that matroidal cones are in the perfect cone decomposition (i.e. $\Sigma_{\text {mat }} \subseteq \Sigma_{P}$ ), establishing that $\Sigma_{P} \cap \Sigma_{V}=\Sigma_{\text {mat }}$. Note in particular that the following special case of [MV12, Thm. A] follows directly from the definitions and Lemma 1.5: if $\sigma \in \Sigma_{\text {mat }}$ is generated by at most $g$ rank 1 quadratic forms, then $\sigma \in \Sigma_{P}$. In particular, if $q \in \sigma \in \Sigma_{P}$ is a rank 1 quadric, then $\mathbb{R}_{\geq 0}\langle q\rangle$ is a face of $\sigma$.

Lemma 1.7 (Central cone). Let $\Lambda$ be a free $\mathbb{Z}$-module of rank $g$. Suppose $\ell_{1}, \ldots, \ell_{n}$ $\in \Lambda^{\vee}$ are primitive, non-zero, linear forms. The following are equivalent:

(1) $\left\{\ell_{1}^{2}, \ldots, \ell_{n}^{2}\right\}$ lie in the same cone of the central cone decomposition.

(2) There exists a quadratic form $Q$ on $\Lambda_{\mathbb{R}}^{\vee}$ such that

(a) $Q(\ell)>0$ for all $\ell \in \Lambda_{\mathbb{R}}^{\vee} \backslash\{0\}$, i.e. $Q$ is positive definite.

(b) $Q(\ell) \geq 1$ for all $\ell \in \Lambda^{\vee} \backslash\{0\}$.

(c) $Q\left(\ell_{i}\right)=1, i=1, \ldots, n$.

(d) $Q(\ell) \in \mathbb{Z}$ for all $\ell \in \Lambda^{\vee}$.

Proof. This follows from the definition of the central cone decomposition in [Nam80]. (See also the proof of [AB12, Thm. 4.8].)

Remark 1.8. We note that all but the last condition above are the same as for the perfect cone compactification, and thus it turns out that if a collection of rank 1 quadratic forms lies in a central cone, they also lie in a perfect cone, but not vice versa (see also Remarks 5.2 and 5.3 below).

Given an admissible cone decomposition $\Sigma$, we will denote by $\Sigma^{(1)}$ the collection of cones that are generated by rank 1 quadrics. Note that if $\sigma \in \Sigma^{(1)}$ and $\tau$ is a face of $\sigma$, then $\tau \in \Sigma^{(1)}$. Note also that by definition $\Sigma_{P}=\Sigma_{P}^{(1)}$. We can summarize the discussion above as follows:

$$
\sigma \in \Sigma_{V}^{(1)}\left(=\Sigma_{\text {mat }}\right) \text { or } \sigma \in \Sigma_{C}^{(1)} \Rightarrow \sigma \in \Sigma_{P}\left(=\Sigma_{P}^{(1)}\right) .
$$


Remark 1.9. The metrics

$$
Q_{A}(\underline{x}):=\sum_{1 \leq i \leq j \leq n} x_{i} x_{j}, \quad Q_{D}(\underline{x}):=\sum_{1 \leq i \leq j \leq n,(i, j) \neq(1,2)} x_{i} x_{j}
$$

define cones of type $A$ and $D$ respectively in the perfect cone decomposition (in fact also in the central cone decomposition). Cones of type $A$ are matroidal, whereas for $n \geq 4$, type $D$ cones are not (and also fail to be simplicial).

Remark 1.10. At this point we recall the relation between the three known admissible decompositions. For $g=\operatorname{rank} \Lambda, g \leq 3$, all three decompositions (namely the second Voronoi, perfect cone and central cone) coincide. For $g=4$ it is still true that the perfect cone and the central cone decompositions coincide, and the second Voronoi decomposition is a refinement of these. More precisely, the only non-basic cone of the perfect cone decomposition, namely the $\mathrm{D}_{4}$ cone, is subdivided into basic cones in the second Voronoi decomposition (see [RE88] for details). For $g=5$ the second Voronoi decomposition is still a refinement of the perfect cone decomposition [RB78], but this is no longer the case for $g \geq 6$ [ER01]. In general, all three decompositions are different in the sense that none is a refinement of other.

\section{Monodromy cones and extensions to toroidal compactifications}

The central question addressed in this paper is that of extending the period map for Prym varieties to toroidal compactifications. The basic set-up for such a problem is that of a locally liftable map $\mathcal{P}: B^{\circ} \rightarrow D / \Gamma$ from a smooth base $B^{\circ}$ to a locally symmetric variety (e.g. maps arising from weight 1 variations of Hodge structure (VHS) associated to families of varieties $\mathfrak{X}^{\circ} / B^{\circ}$ ). We then consider a partial simple normal crossing smooth compactification $B^{\circ} \subset B$ and we are asking about extensions of the map $\mathcal{P}$ from $B$ to a given (fixed) toroidal compactification $\overline{D / \Gamma} \Gamma^{\Sigma}$. Since the problem is essentially local, we may assume without loss of generality that $B^{\circ}$ is a polycylinder (i.e. $B^{0}=\left(S^{\circ}\right)^{k} \times S^{n-k} \subset$ $B=S^{n}$, where $S^{\circ}=S \backslash\{0\}$ and $S$ is the unit disk), and that the monodromy operators around the boundary divisors are unipotent.

With this set-up the extension question has an elegant answer. Namely, one defines a monodromy cone associated to the period map $\mathcal{P}$, and then $\mathcal{P}$ extends if and only if the monodromy cone is compatible with the cones of the admissible decomposition $\Sigma$. We review this below, following Cattani [Cat84], with a focus on weight 1 variations of Hodge structures (although some of the considerations apply more generally).

\subsection{Degenerations of weight 1 Hodge structures}

The monodromy cone for a variation of Hodge structures is a basic tool in understanding extensions of period maps. Here we review the definition of the log of monodromy, the monodromy cone, and the connection with quadratic forms. 
2.1.1. The log of monodromy. We focus on the case of weight 1 Hodge structures for simplicity. Let $\pi^{\circ}: \mathfrak{X}^{\circ} \rightarrow S^{\circ}$ be a smooth, projective morphism over the punctured disk $S^{\circ}$. Fix a base point $* \in S^{\circ}$, with fiber $X_{*}=\left(\pi^{\circ}\right)^{-1}(*)$, and let $T$ be the associated monodromy operator on $H^{1}\left(X_{*}, \mathbb{Q}\right)$. It is well known that $T$ is quasi-unipotent; in fact, since we are in weight 1 , we have $\left(T^{n}-\mathrm{Id}\right)^{2}=0$. For simplicity, we will assume further that $T$ is in fact unipotent, i.e. $(T-\mathrm{Id})^{2}=0$. Since unipotent monodromy can be obtained after a finite base change, this assumption will not affect extension questions (see Remark 2.5). Thus

$$
N=\log T=T-\operatorname{Id} \in \operatorname{End} H^{1}\left(X_{*}, \mathbb{Q}\right)
$$

is the log of monodromy operator. Note that $N \in \mathfrak{s p}(H, Q)$, where $H=H^{1}\left(X_{*}, \mathbb{Q}\right)$ and $Q$ is the intersection pairing on $H$, and $N^{2}=0$.

To relate this to the discussion of Section 1, we would like to view $N$ as a quadratic form. To this end we recall that there is a limit polarized, mixed Hodge structure $H_{\lim }^{1}=H_{\lim }^{1}(N)$ on the torsion free quotient $H^{1}\left(X_{*}, \mathbb{Z}\right)_{\tau}$, where the weight filtration $W_{\bullet}=W_{\bullet}(N)$ is defined (using $N^{2}=0$ ) by

$$
W_{-1}=\{0\} \subset W_{0}=\operatorname{Im}(N) \subseteq W_{1}:=\operatorname{ker}(N) \subseteq W_{2}:=H^{1}\left(X_{*}, \mathbb{Q}\right) .
$$

As in (1.3) and (1.4) (which are essentially linear algebra statements about nilpotent symplectic endomorphisms), we can view the log of monodromy as a map

$$
\begin{aligned}
Q(N(\cdot), \cdot): \operatorname{Gr}_{2}(N) & \rightarrow\left(\operatorname{Gr}_{2}(N)\right)^{\vee} \in \operatorname{Hom}\left(\operatorname{Sym}^{2} \operatorname{Gr}_{2}(N), \mathbb{Q}\right), \\
\bar{z} & \mapsto Q(N(\cdot), z),
\end{aligned}
$$

or equivalently as a symmetric bilinear form on $\mathrm{Gr}_{2}(N)$.

Remark 2.1. Since we will need to explicitly compute monodromy in several cases, we note that with respect to a suitable symplectic basis on $H^{1}\left(X_{*}\right)$, we can write (e.g. [Nam80, Prop. 4.8])

$$
T=\left(\begin{array}{cccc}
1_{g^{\prime}} & 0 & 0 & 0 \\
0 & 1_{v} & 0 & b \\
0 & 0 & 1_{g^{\prime}} & 0 \\
0 & 0 & 0 & 1_{v}
\end{array}\right), \quad N=\log T=\left(\begin{array}{llll}
0 & 0 & 0 & 0 \\
0 & 0 & 0 & b \\
0 & 0 & 0 & 0 \\
0 & 0 & 0 & 0
\end{array}\right),
$$

with $b$ a symmetric non-degenerate $v \times v$ matrix, $v=\operatorname{dim} W_{0}=\operatorname{Im}(N)$ and $g^{\prime}=$ $g-v$. The identification of $N$ with a quadratic form is simply obtained by considering the matrix $b$. The salient point of the discussion above is that $b$ should be viewed as a quadratic form on $\mathrm{Gr}_{2}(N)$, which is essential for compatibility issues as discussed below.

Remark 2.2. To a 1-parameter unipotent degeneration of weight 1 Hodge structures, one can associate either a limit mixed Hodge structure (MHS) (from the point of view of degenerations of Hodge structures following Schmid [Sch73] and Steenbrink [Ste76]) or a semiabelian variety (see \$2.4). The two limit objects are canonically identified via the functorial equivalence of categories between semiabelian varieties and polarized weight 1 
MHS (e.g. Deligne [Del74, §10]). From the perspective of monodromy matrices discussed above, the $g^{\prime} \times g^{\prime}$ blocks correspond to the compact part of the limit semiabelian variety and are essentially irrelevant to the extension question. On the other hand, the $v \times v$ matrix $b$ defining the quadratic form is a key ingredient for extension questions.

2.1.2. Monodromy cones. We now consider families over higher-dimensional bases. Let $\pi^{\circ}: \mathfrak{X}^{\circ} \rightarrow\left(S^{\circ}\right)^{k} \times S^{n-k}$ be a smooth, projective morphism. Fix a base point $* \in\left(S^{\circ}\right)^{n}$, with fiber $X_{*}=\left(\pi^{\circ}\right)^{-1}(*)$, and let $T_{i}(i=1, \ldots, k)$ be the associated monodromy operators on $H^{1}\left(X_{*}, \mathbb{Q}\right)$, i.e. generators for the induced homomorphism $\mathbb{Z}^{k} \cong \pi_{1}\left(\left(S^{\circ}\right)^{k}, *\right) \rightarrow$ Aut $H^{1}\left(X_{*}, \mathbb{Q}\right)$. For simplicity, as before, we will assume further that the $T_{i}$ are in fact unipotent, and let $N_{i}=\log T_{i}=T_{i}-\operatorname{Id}(i=1, \ldots, k)$ be the $\log$ of monodromy operators. Again, since this can be obtained after finite base change, this will not affect extension questions. We can now define the monodromy cone

$$
\sigma\left(\pi^{\circ}\right):=\mathbb{R}^{+}\left\langle N_{1}, \ldots, N_{k}\right\rangle \subseteq \mathfrak{s p}\left(H_{\mathbb{R}}, Q\right),
$$

with $H=H^{1}\left(X_{*}, \mathbb{Q}\right)$ and $Q$ the intersection pairing on $H$.

As before, we would like to identify this cone with a cone of quadratic forms on a fixed vector space. The point is that for each $\lambda_{1}, \ldots, \lambda_{k}>0$, we obtain a limit mixed Hodge structure $H_{\mathrm{lim}}^{1}(\underline{\lambda})=H_{\lim }^{1}\left(\sum_{i=1}^{k} \lambda_{i} N_{i}\right)$ on $H^{1}\left(X_{*}, \mathbb{Q}\right)$, with monodromy weight filtration $W_{\bullet}(\underline{\lambda})=W_{\bullet}\left(\sum_{i=1}^{k} \lambda_{i} N_{i}\right)$ given by (2.2) with

$$
N=\lambda_{1} N_{1}+\cdots+\lambda_{k} N_{k}
$$

It is well known (see e.g. [Cat84]) that for $\lambda_{1}, \ldots, \lambda_{k}>0$,

$$
\operatorname{ker}\left(\lambda_{1} N_{1}+\cdots+\lambda_{k} N_{k}\right)=\bigcap_{i=1}^{k} \operatorname{ker}\left(N_{i}\right) .
$$

Thus $W_{1}(\underline{\lambda})$, and hence $\mathrm{Gr}_{2}(\underline{\lambda})$, is independent of the $\lambda_{i}>0$. Consequently, for $\lambda_{1}, \ldots, \lambda_{k}$ $>0$, the $\lambda_{1} N_{1}+\cdots+\lambda_{k} N_{k}$ can all be viewed as quadratic forms on

$$
\mathrm{Gr}_{2}:=H^{1}\left(X_{*}, \mathbb{Q}\right) / \bigcap \operatorname{ker}\left(N_{i}\right) \text {. }
$$

In conclusion, the monodromy cone can be identified with a cone of symmetric matrices on the vector space $\mathrm{Gr}_{2}$,

$$
\sigma\left(\pi^{\circ}\right):=\mathbb{R}^{+}\left\langle N_{1}, \ldots, N_{k}\right\rangle \subseteq \operatorname{Hom}\left(\operatorname{Sym}^{2} \mathrm{Gr}_{2}, \mathbb{R}\right) .
$$

Remark 2.3. Using $N^{2}=0$ and the symplectic form $Q$, we obtain a natural identification $W_{0}=W_{1}^{\perp}$, which then gives an identification

$$
W_{0}(N)=\operatorname{Im}(N)=\sum \operatorname{Im}\left(N_{i}\right)
$$

2.1.3. Closures of monodromy cones. We now discuss the closure of the monodromy cone. Clearly in regard to the description (2.4), we have

$$
\overline{\sigma\left(\pi^{\circ}\right)}=\mathbb{R}_{\geq 0}\left\langle N_{1}, \ldots, N_{k}\right\rangle \subseteq \mathfrak{s p}\left(H_{\mathbb{R}}, Q\right) .
$$


However, in regard to (2.6), the description is not as obvious. The issue is that if we set

$$
\mathrm{Gr}_{2}=\mathrm{Gr}_{2}(\underline{\lambda})=H^{1}\left(X_{*}, \mathbb{Q}\right) / \bigcap \operatorname{ker}\left(N_{i}\right)
$$

the $N_{i}$ individually are not naturally identified as quadratic forms in $\operatorname{Hom}\left(\operatorname{Sym}^{2} \mathrm{Gr}_{2}, \mathbb{R}\right)$; they are quadratic forms in $\operatorname{Hom}\left(\operatorname{Sym}^{2} \operatorname{Gr}_{2}\left(W_{\bullet}\left(N_{i}\right)\right), \mathbb{R}\right)$, respectively. To remedy this, set $\bar{N}_{i}(i=1, \ldots, k)$ to be the composition

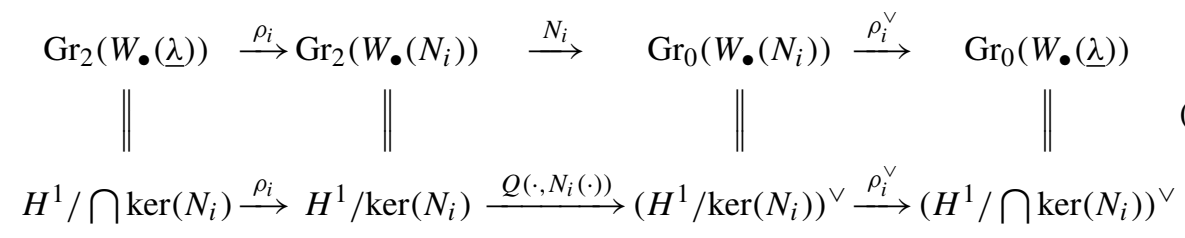

Then unwinding the definitions, we obtain

$$
\overline{\sigma\left(\pi^{\circ}\right)}=\mathbb{R}_{\geq 0}\left\langle\bar{N}_{1}, \ldots, \bar{N}_{k}\right\rangle \subseteq \operatorname{Hom}\left(\operatorname{Sym}^{2} \mathrm{Gr}_{2}, \mathbb{R}\right) .
$$

\subsection{Monodromy cones in the geometric context}

In the previous subsection we have discussed the abstract Hodge-theoretic aspects associated to a degeneration. This discussion allows us to tie in with the theory of toroidal compactifications discussed in Section 1. Further, via the discussion of $\S 2.1 .3$, it reduces the computation of monodromy cones to the case of 1-parameter bases. Here we assume that this 1-parameter VHS arises from a 1-parameter geometric family. In this situation, we would like to interpret the monodromy cones in terms of the geometry and combinatorics of the central fiber (i.e. the limit geometric object).

Namely, we assume here that there is a smooth family $\pi^{\circ}: \mathfrak{X}^{\circ} \rightarrow S^{\circ}$ which has an extension $\pi: \mathfrak{X} \rightarrow S$ to a projective morphism, with central fiber $X_{0}=\pi^{-1}(0)$ a simple normal crossing divisor in the family (this can be obtained after a finite base change by the semistable reduction theorem, and will not affect extension questions). This will also imply that the monodromy is unipotent. As is well known, the central fiber $X_{0}$ carries a canonical mixed Hodge structure. Furthermore, the Clemens-Schmid exact sequence (e.g. [Mor84, p. 109]) relates the limit mixed Hodge structure on $X_{*}$ to the MHS on $X_{0}$. To recall the sequence we let $i: X_{*} \rightarrow X_{0}$ be the Clemens collapsing map (the composition of the inclusion $X_{*} \subseteq \mathfrak{X}$ with the contraction $\mathfrak{X} \rightarrow X_{0}$ ), and we will denote by PD any of the Poincare duality isomorphisms. In the weight 1 case, the Clemens-Schmid exact sequence is

$$
0 \rightarrow H^{1}\left(X_{0}\right) \stackrel{i^{*}}{\rightarrow} H_{\lim }^{1}\left(X_{*}\right) \stackrel{N}{\rightarrow} H_{\lim }^{1}\left(X_{*}\right) \stackrel{\beta=\iota_{*} \mathrm{PD}}{\rightarrow} H_{1}\left(X_{0}\right) \stackrel{\alpha}{\rightarrow} H^{3}\left(X_{0}\right) \stackrel{i^{*}}{\rightarrow} \cdots
$$

Since we will not use the definition of $\alpha$, we refer the reader to [Mor84, p.108]. The maps $\alpha, i^{*}, N$, and $\beta$ are morphisms of mixed Hodge structures of types $(2,2),(0,0)$, $(-1,-1)$, and $(-1,-1)$ respectively. It follows that there are isomorphisms

$$
H^{1}(\Gamma, \mathbb{Q})=\mathrm{Gr}_{0}^{X_{0}}\left(H^{1}\left(X_{0}\right)\right) \stackrel{i^{*}}{\rightarrow} \mathrm{Gr}_{0}
$$


(the first identification being given by the Mayer-Vietoris spectral sequence for $X_{0}$ ) and

$$
\mathrm{Gr}_{2} \stackrel{\beta=i_{*} \mathrm{PD}}{\longrightarrow} \operatorname{Gr}_{0}^{X_{0}}\left(H_{1}\left(X_{0}\right)\right)=\frac{\left(W_{-1}\left(H^{1}\left(X_{0}\right)\right)\right)^{\perp}}{\left(W_{0}\left(H^{1}\left(X_{0}\right)\right)\right)^{\perp}}=\left(\mathrm{Gr}_{0}^{X_{0}}\left(H^{1}\left(X_{0}\right)\right)\right)^{\vee}
$$

Thus composing, we may view the log of monodromy as a map

$$
\left(\mathrm{Gr}_{0}^{X_{0}}\left(H^{1}\left(X_{0}\right)\right)\right)^{\vee} \stackrel{\beta^{-1}}{\longrightarrow} \mathrm{Gr}_{2} \stackrel{N}{\rightarrow} \mathrm{Gr}_{0} \stackrel{\left(\iota^{*}\right)^{-1}}{\longrightarrow} H^{1}(\Gamma, \mathbb{Q})
$$

Again using the identification $\operatorname{Gr}_{0}^{X_{0}}\left(H^{1}\left(X_{0}\right)\right)=H^{1}(\Gamma, \mathbb{Q})$, we may identify the spaces $H^{1}(\Gamma, \mathbb{Q})^{\vee}=H_{1}(\Gamma, \mathbb{Q})$ by the universal coefficients theorem, and the composition (2.10) allows us to view the log of monodromy as a map

$$
N: H_{1}(\Gamma, \mathbb{Q}) \rightarrow H^{1}(\Gamma, \mathbb{Q}) \in \operatorname{Hom}\left(\operatorname{Sym}^{2} H_{1}(\Gamma, \mathbb{Q}), \mathbb{Q}\right) .
$$

Consequently, for the case of a family of stable curves $\pi: \mathfrak{X} \rightarrow S^{n}$, smooth over $\left(S^{\circ}\right)^{k} \times S^{n-k}$, the monodromy cone is given by

$$
\sigma\left(\pi^{\circ}\right):=\mathbb{R}^{+}\left\langle N_{1}, \ldots, N_{k}\right\rangle \subseteq \operatorname{Hom}\left(\operatorname{Sym}^{2} H_{1}(\Gamma, \mathbb{Q}), \mathbb{Q}\right) \mathbb{R}
$$

where $\Gamma$ is the dual graph to the curve $X_{0}=\pi^{-1}(0)$, and $N_{i}$ is the log of monodromy around the hyperplane $\left\{x_{i}=0\right\}$.

In order to describe the closure of the monodromy cone, we introduce some further notation. Let $0 \neq 0_{i} \in S^{n}$ be a point in the hyperplane $\left\{x_{i}=0\right\}$, sufficiently close to 0 . Let $X_{0_{i}}$ be the fiber over $0_{i}$, and let $\Gamma_{i}$ be the dual graph of $X_{0_{i}}$. The problem with describing the closure of the monodromy cone is that $N_{i}$ is not a quadratic form on $H_{1}(\Gamma, \mathbb{Q})$, rather it is a quadratic form on $H_{1}\left(\Gamma_{i}, \mathbb{Q}\right)$. We resolve this using $(2.8)$, and the identification $\beta: \mathrm{Gr}_{2} \rightarrow H_{1}(\Gamma, \mathbb{Q})$. Thus, there exist morphisms

$$
H_{1}(\Gamma, \mathbb{Q}) \stackrel{\rho_{i}}{\rightarrow} H_{1}\left(\Gamma_{i}, \mathbb{Q}\right)
$$

such that if we let $\bar{N}_{i}$ be the composition

$$
H_{1}(\Gamma, \mathbb{Q}) \stackrel{\rho_{i}}{\rightarrow} H_{1}\left(\Gamma_{i}, \mathbb{Q}\right) \stackrel{N_{i}}{\rightarrow} H^{1}\left(\Gamma_{i}, \mathbb{Q}\right) \stackrel{\rho_{i}^{\vee}}{\rightarrow} H^{1}(\Gamma, \mathbb{Q}),
$$

then the closure of the monodromy cone is given by

$$
\overline{\sigma\left(\pi^{\circ}\right)}=\mathbb{R}_{\geq 0}\left\langle\bar{N}_{1}, \ldots, \bar{N}_{k}\right\rangle \subseteq \operatorname{Hom}\left(\operatorname{Sym}^{2} H_{1}(\Gamma, \mathbb{Q}), \mathbb{Q}\right)_{\mathbb{R}} .
$$

Finally, the map $\rho_{i}$ in (2.13) can be described combinatorially. For each $j=1, \ldots, k$ there is a natural map of chain complexes $C_{\bullet}(\Gamma, \mathbb{Z}) \rightarrow C_{\bullet}\left(\Gamma_{j}, \mathbb{Z}\right)$ (see Appendix D, where $\Gamma_{j}$ is denoted $\left.\Gamma / S^{c}\right)$, inducing surjective maps

$$
H_{1}(\Gamma, \mathbb{Q}) \rightarrow H_{1}\left(\Gamma_{j}, \mathbb{Q}\right) .
$$

We claim that this map agrees with the map $\rho_{j}$ above. This follows from the definitions, and we sketch the argument here. The key point is the identification

$$
\beta=i_{*} \mathrm{PD}: \mathrm{Gr}_{2}:=H_{\mathrm{lim}}^{1} / W_{1} \rightarrow \operatorname{Gr}_{0}^{X_{0}}\left(H_{1}\right):=H_{1}\left(X_{0}\right) / W_{-1}^{X_{0}}\left(H_{1}\right) .
$$


We define $W_{-1}^{X_{0}}\left(H_{1}\right)=\left(W_{0}^{X_{0}}\left(H^{1}\right)\right)^{\perp}$. Dualizing the exact sequence (obtained from the Mayer-Vietoris spectral sequence)

$$
0 \rightarrow W_{0}^{X_{0}}\left(H^{1}\right) \rightarrow H^{1}\left(X_{0}\right) \rightarrow H^{1}\left(\widehat{X}_{0}\right) \rightarrow 0
$$

we find that $\left(W_{0}^{X_{0}}\left(H^{1}\right)\right)^{\perp}=H_{1}\left(\widehat{X}_{0}\right)$ using the universal coefficients theorem, where $\widehat{X}_{0}$ denotes the normalization of $X_{0}$. In short, we have

$$
\beta=i_{*} \mathrm{PD}: \mathrm{Gr}_{2} \rightarrow H_{1}\left(X_{0}\right) / H_{1}\left(\widehat{X}_{0}\right) .
$$

Thus there is a commutative diagram

$$
\begin{array}{cc}
\operatorname{Gr}_{2}\left(\sum \lambda_{j} N_{j}\right)=H^{1}\left(X_{*}\right) / \bigcap \operatorname{ker}\left(N_{j}\right) \stackrel{\rho}{\operatorname{Gr}_{2}\left(N_{j}\right)=H^{1}\left(X_{*}\right) / \operatorname{ker}\left(N_{j}\right)} \\
\beta=i_{*} P D \downarrow & \beta=i_{*} P D \downarrow \\
H_{1}(\Gamma)=H^{1}(\Gamma)^{\vee}=H_{1}\left(X_{0}\right) / H_{1}\left(\widehat{X}_{0}\right) \stackrel{\left(i_{*} \mathrm{PD}\right)\left(\mathrm{PD}^{-1} i_{*}^{-1}\right)}{\longrightarrow} H_{1}\left(X_{0_{j}}\right) / H_{1}\left(\widehat{X}_{0_{j}}\right)=H^{1}\left(\Gamma_{j}\right)^{\vee}=H_{1}\left(\Gamma_{j}\right)
\end{array}
$$

The bottom row is easily seen to be the combinatorial map (2.16) above (note that $i_{*}^{-1}$ is only defined up to vanishing cycles, but nevertheless, $i_{*} i_{*}^{-1}$ is well defined as a map on the quotients).

Remark 2.4. One can also identify $\rho_{j}^{\vee}$ directly. Following the definitions, one finds that it is given by

$$
\begin{aligned}
H^{1}\left(\Gamma_{j}\right) & =\operatorname{Gr}_{0}\left(H^{1}\left(X_{0}\right)\right) \stackrel{i^{*}}{\cong} \operatorname{Gr}_{0}\left(N_{j}\right)=\operatorname{Im}\left(N_{j}\right) \hookrightarrow \operatorname{Im}\left(\sum N_{j}\right) \\
& =\operatorname{Im}(N)=\operatorname{Gr}_{0}(N) \stackrel{\left(i^{*}\right)^{-1}}{\cong} H^{1}(\Gamma) .
\end{aligned}
$$

This can also be identified with the dual of the combinatorial map given above.

\subsection{Monodromy cones and extensions of period maps}

Now that we have defined the pertinent terms, we can discuss the standard extension results for period maps to toroidal compactifications. We begin by making one remark.

Remark 2.5. Let $B$ be a smooth variety. It is a basic fact that if a rational map from $B$ to any of the compactifications of the moduli of abelian varieties extends after a finite base change, then the rational map itself extends. For this reason, we will be free in what follows to make finite base changes when considering extensions of period maps.

2.3.1. Extension via Hodge theory. Fix a compatible collection of admissible cone decompositions $\Sigma$, and let $\bar{A}_{g}^{\Sigma}$ be the associated toroidal compactification. Let

$$
f^{\circ}:\left(S^{\circ}\right)^{k} \times S^{n-k} \rightarrow A_{g}
$$

be a locally liftable morphism (i.e. one induced by a family of abelian varieties). After a finite base change, we may assume that the monodromy operators $T_{i}$ around the boundary 
divisors $\left\{x_{i}=0\right\}$ are unipotent (see Remark 2.5). Then setting $N_{i}=\log T_{i}$, we see that for any $\lambda_{1}, \ldots, \lambda_{k}>0$, there is a fixed $Q$-isotropic subspace $W_{0}=\operatorname{ker}\left(\sum_{i} \lambda_{i} N_{i}\right)$. The Borel extension theorem [Bor72] implies that $f^{\circ}$ extends to a morphism $f: S^{n} \rightarrow A_{g}^{*}$. The isotropic subspace $W_{0}$ determines a boundary component $F_{W_{0}}$, and in turn a boundary component $A_{g^{\prime}}^{*}$. The point $f(0)$ is the point of $A_{g^{\prime}}^{*}$ associated to the pure weight 1 Hodge structure determined by the first graded piece of the limit mixed Hodge structure of any semistable reduction of the restriction of the (induced) family (of say abelian varieties) to a 1-parameter base. The following extension theorem is well known (see e.g. [Nam80, Thm. 7.29, Rem. 7.30], [AM+10, Thm. 7.2], [FC90, Thm. 5.7, p. 116]):

Fact 2.6. The map $f^{\circ}$ extends to a morphism $S^{n} \rightarrow \bar{A}_{g}^{\Sigma}$ if and only if the monodromy cone (as defined in (2.6)) is contained in a cone in $\Sigma$ (more specifically, a cone in $\Sigma_{W_{0}}$ ).

2.3.2. Resolving period maps. We now recall how one can resolve the period map to a toroidal compactification $\bar{A}_{g}^{\Sigma}$ of the moduli of abelian varieties. Let us assume that we have a semiabelian scheme $\mathscr{X}^{g} \rightarrow B$ where $B$ is smooth, and that there is a simple normal crossing divisor $\Delta \subseteq B$ such that the family is abelian over $B^{\circ}=B-\Delta$. Fix a base point $0 \in \Delta$. The variety $(B, \Delta)$ is toroidal at 0 , corresponding to the semigroup $\operatorname{ring} \mathbb{C}\left[\mathbb{N}^{k}\right]$, where $k$ is the number of components of $\Delta$ meeting at 0 . Fix a basis $e_{1}, \ldots, e_{k}$ for $\mathbb{R}^{k}$. We say that $\mathbb{R}_{\geq 0}\left\langle e_{i}\right\rangle$ is the cone associated to the toric data at 0 . Now let $\sigma(\mathscr{X} / B)$ be the monodromy cone associated to the semiabelian family at 0 . The period map defines a map of cones

$$
\mu: \mathbb{R}_{\geq 0}\left\langle e_{i}\right\rangle \rightarrow \sigma(\mathscr{X} / B), \quad e_{i} \mapsto \log T_{e_{i}},
$$

where $T_{e_{i}}$ is the monodromy around the hyperplane associated to $e_{i}$. Now the admissible decomposition $\Sigma$ decomposes $\sigma(\mathscr{X} / B)$ into a fan $\mathscr{F}_{0}^{\Sigma}$ of cones.

Fact 2.6 states that the period map extends at 0 if and only if $\mathscr{F}_{0}^{\Sigma}$ has just one cone of maximal dimension. Otherwise, there is an induced fan $\mu^{-1} \mathscr{F}_{0}^{\Sigma}$ decomposing the cone $\mathbb{R}_{\geq 0}\left\langle e_{i}\right\rangle$. Any fan $\mathscr{F}$ decomposing $\mathbb{R}_{\geq 0}\left\langle e_{i}\right\rangle$ determines a birational morphism to $\mathbb{A}_{\mathbb{C}}^{k}$. Any fan $\mathscr{F}$ that refines $\mu^{-1} \mathscr{F}_{0}^{\Sigma}$ determines a birational modification of $B$ (in an étale neighborhood of 0) that resolves the period map in an (étale) neighborhood of 0 . In particular, the fan $\mu^{-1} \mathscr{F}_{0}^{\Sigma}$ determines the minimal toric birational modification that will resolve the period map. This minimal toric birational modification is canonical and glues to give a birational modification for a period map on a moduli space.

\subsection{Moduli stacks and abelian varieties}

We may also view the toroidal compactifications as compactifications of the moduli of abelian varieties. We begin by recalling the basic structure of toroidal compactifications from this perspective. Every toroidal compactification $A_{g}^{\Sigma}$ has a canonical map

$$
\varphi^{\Sigma}: A_{g}^{\Sigma} \rightarrow A_{g}^{*}=A_{g} \sqcup A_{g-1} \sqcup \cdots \sqcup A_{0}
$$

which defines a stratification $\beta_{i}^{\Sigma}=\left(\varphi^{\Sigma}\right)^{-1}\left(A_{g-i}\right)$. The strata $\beta_{i}^{\Sigma}$ are themselves stratified, $\beta_{i}^{\Sigma}=\bigsqcup \beta(\sigma)$ where $\sigma$ runs through all $\mathrm{GL}(i, \mathbb{Z})$-orbits of cones in the decompo- 
sition $\Sigma$ of $\operatorname{Sym}^{2}\left(\mathbb{Z}^{i}\right)$ containing rank $i$ matrices. The strata $\beta(\sigma)$ themselves are of the form $\beta(\sigma)=\mathcal{T}(\sigma) / \mathcal{G}(\sigma)$ where $\mathcal{T}(\sigma)$ is a torus bundle over the $i$-fold fiber product of the universal family $p: \mathcal{X}_{g-i} \rightarrow \mathcal{A}_{g-i}$. Indeed, $\pi(\sigma)=p^{\times i} \circ q(\sigma): \mathcal{T}(\sigma) \rightarrow \mathcal{X}_{g-i}^{\times i} \rightarrow$ $\mathcal{A}_{g-i}$ where $q(\sigma)$ is a torus bundle whose fibers have dimension $i(i+1) / 2-\operatorname{dim}(\sigma)$. More precisely, $\mathcal{T}(\sigma)=\mathcal{T}_{i} / \mathcal{T}_{\sigma}$ where the fibers of $\mathcal{T}_{i}$ and $\mathcal{T}_{\sigma}$ are $\left(\mathrm{Sym}^{2} \mathbb{Z}^{i}\right) \otimes \mathbb{C}^{*}$ and $\left(\langle\sigma\rangle \cap \operatorname{Sym}^{2} \mathbb{Z}^{i}\right) \otimes \mathbb{C}^{*}$ respectively. The group $G(\sigma)$ is the stabilizer of the cone $\sigma$ in $\mathrm{GL}(i, \mathbb{Z})$ and acts naturally on $\mathcal{T}(\sigma)$. The codimension of $\mathcal{T}(\sigma)$ in $A_{g}^{\Sigma}$ is $\operatorname{dim}(\sigma)$.

2.4.1. The Faltings-Chai stacks. Faltings-Chai have given a stack-theoretic interpretation of the toroidal compactifications. For each toroidal compactification $\bar{A}_{g}^{\Sigma}$, there is an irreducible, normal, proper, Deligne-Mumford $\mathbb{C}$-stack $\overline{\mathcal{A}}_{g}^{\Sigma}$ with coarse moduli space $\bar{A}_{g}^{\Sigma}$, and a semiabelian scheme $\mathcal{X}_{g}^{\Sigma} \rightarrow \overline{\mathcal{A}}_{g}^{\Sigma}$ extending the universal abelian variety $\mathcal{X}_{g} \rightarrow \mathcal{A}_{g}$ [FC90, Thm. 5.7(5), p. 117]. While the stack does not represent a moduli functor of semiabelian varieties, we do have the following. A semiabelian scheme $X \rightarrow S$ over the disk such that the restriction $X^{\circ} \rightarrow S^{\circ}$ to the punctured disk is abelian (i.e. a morphism $S \rightarrow \overline{\mathcal{A}}_{g}^{\Sigma}$ with $S^{\circ} \rightarrow \mathcal{A}_{g}$ ) is determined by a set of degeneration data (see also [Ale02], [ABH02]), namely:

(D0) A principally polarized abelian variety $(A, M)$ (with $M$ an ample line bundle) inducing an isomorphism $\lambda_{M}: A \rightarrow \widehat{A}$, where $\widehat{A}=\operatorname{Pic}^{0}(A)$.

(D1a) A semiabelian variety $0 \rightarrow \mathbb{T} \rightarrow G \rightarrow A \rightarrow 0$ with split torus part, determined by a homomorphism $c: \Lambda \rightarrow \widehat{A}$, where $\Lambda$ is the character lattice of $\mathbb{T}$.

(D1b) A second semiabelian variety $0 \rightarrow \widehat{\mathbb{T}} \rightarrow \widehat{G} \rightarrow \widehat{A} \rightarrow 0$ induced by a homomorphism $\hat{c}: \widehat{\Lambda} \rightarrow A$, where $\widehat{\Lambda}$ is the character lattice of $\widehat{\mathbb{T}}$.

(D2) An isomorphism of lattices $\phi: \widehat{\Lambda} \rightarrow \Lambda$ such that $c \circ \phi=\lambda_{M} \circ \hat{c}$.

(D3) A bihomomorphism $\tau: \widehat{\Lambda} \times \Lambda \rightarrow(\hat{c} \times c)^{*}\left(\mathscr{P}^{\circ}\right)^{-1}$, where $\mathscr{P}^{\circ}$ is the rigidified Poincaré bundle with the zero section removed.

(D4) A cubical morphism $\psi: \widehat{\Lambda} \rightarrow \hat{c}^{*}\left(M^{\circ}\right)^{-1}$, where $M^{\circ}$ is the principal bundle obtained from removing the zero section of $M$.

(D5) For each $\lambda \in \Lambda$ a section $\theta_{\lambda} \in \Gamma(A, M \otimes c(\lambda))$ satisfying some further compatibility conditions with the data above. This data, more precisely the bihomomorphism $\tau$, defines a quadratic form $B: \Lambda \times \Lambda \rightarrow \mathbb{Q}$, which in fact agrees with the $\log$ of monodromy for the 1-parameter family (at least up to GL-conjugation and scaling, which is irrelevant from the extension of period maps perspective).

(D6) The Delaunay decomposition of $\Lambda_{\mathbb{R}}$ determined by $B$.

Given this data, we can describe the image of the central point under the map $f: S \rightarrow \bar{A}_{g}^{\Sigma}$ as follows. The data (D0) determines the image of 0 under the composition $S \rightarrow \bar{A}_{g}^{\Sigma}$ $\rightarrow A_{g}^{*}$; in particular it determines the stratum $\beta_{i}$ described above, in which $f(0)$ lies. The quadratic form $B$ lies in a unique cone $\sigma \in \Sigma$ of minimal dimension. The point $f(0)$ then lies in the stratum $\beta(\sigma) \subseteq \beta_{i}$, described above. The remaining degeneration data determines the specific point $f(0)$ within $\beta(\sigma)$. More precisely, using the description of $\mathcal{T}_{i}$ given in [GHT15, Prop. 7.2] the bihomomorphism $\tau$ defines a point in $\mathcal{T}_{i}$, and $f(0)$ is its image in $\beta^{V}(\sigma)=\left(\mathcal{T}_{i} / \mathcal{T}_{\sigma}\right) / G(\sigma)$. 
Remark 2.7. Let $\overline{\mathcal{M}}$ be a smooth Deligne-Mumford $\mathbb{C}$-stack containing an open substack $\mathcal{M}$ with normal crossing boundary divisor $\Delta=\overline{\mathcal{M}} \backslash \mathcal{M}$. Let $\bar{M}$ and $M$ be the respective coarse moduli spaces. Suppose there exists a morphism $\mathcal{M} \rightarrow \mathcal{A}_{g}$ inducing a morphism $M \rightarrow A_{g}$. By the Abramovich-Vistoli purity lemma, the morphism $\mathcal{M} \rightarrow \mathcal{A}_{g}$ extends to a morphism $\overline{\mathcal{M}} \rightarrow \overline{\mathcal{A}}_{g}^{\Sigma}$ if and only if the morphism $M \rightarrow A_{g}$ extends to a morphism $\bar{M} \rightarrow \bar{A}_{g}^{\Sigma}$.

Remark 2.8. The singularities of the stack $\overline{\mathcal{A}}_{g}^{\Sigma}$ can be read off from the cones in $\Sigma$. Basic cones give rise to smooth points of the stack. Simplicial but non-basic cones correspond to quotient singularities by finite abelian groups. More precisely, these groups are identified with the quotient of the lattice $\left(\operatorname{Sym}^{2} \Lambda\right)^{\vee} \cap\langle\sigma\rangle$ by the sublattice generated by the integral generators of $\sigma$. Non-simplicial cones give rise to more general (toric) singularities of the moduli stack. Singularities of the varieties $\bar{A}_{g}^{\Sigma}$ can also occur if the cones are basic, in fact they already occur on $A_{g}$ itself. The singularities depend on the finite stabilizer of a point in the toric construction. The codimension of the singular locus of the stack $\overline{\mathcal{A}}_{g}^{P}$ is 10 , whereas it is 3 in the case of $\overline{\mathcal{A}}_{g}^{V}$ [DHS13].

2.4.2. Alexeev's stack of stable semiabelic pairs. Alexeev has constructed a moduli space $\overline{\mathcal{A}}_{g}^{A}$ of complex stable semiabelic pairs that contains $\mathcal{A}_{g}$ as an open substack [Ale02]. The stack $\overline{\mathcal{A}}_{g}^{A}$ is a proper, algebraic (Artin) $\mathbb{C}$-stack with finite diagonal [Ale02, Thm. 5.10.1]. Moreover, the stack admits a coarse moduli space, with a component that has normalization isomorphic to the second Voronoi compactification $\bar{A}_{g}^{V}$ [Ale02, Thm. 5.11.6, p. 701] (see also Olsson [Ols08]). The main point is that the degeneration data described above define locally relatively complete models which admit an action of the universal semiabelian variety. Gluing these to obtain a universal family over a compactification of $\mathcal{A}_{g}$, one is naturally led to the second Voronoi decomposition.

We also recall how the degeneration data above determine a stable semiabelic pair. The Delaunay decomposition defines a fan on $\mathbb{R} \oplus \Lambda_{\mathbb{R}}$ with cones determined by the Delaunay decomposition shifted by $(1,0) \in \mathbb{N} \oplus \Lambda$. We use this to define an $\mathscr{O}_{A}$-algebra $\mathscr{R}$. As a module, $\mathscr{R}$ is freely generated by $M_{\chi}:=M^{\otimes d} \otimes c(\lambda)$ for each $\chi=(d, \lambda) \in \mathbb{N} \oplus \Lambda$. We define multiplication by the natural identification $M_{\chi_{1}} \otimes M_{\chi_{2}}=M_{\chi_{1}+\chi_{2}}$ when $\chi_{1}, \chi_{2} \in \delta$ lie in a common cone in the fan over the Delaunay decomposition. When $\chi_{1}, \chi_{2}$ do not lie in a common cone, sections multiply to 0 . The morphisms $\tau$ and $\phi$ define a natural action of $\widehat{\Lambda}$ on $\mathscr{R}$, and the action is properly discontinuous in the Zariski topology on the relative Proj, so that $X=\left(\operatorname{Proj}_{A} \mathscr{R}\right) / \widehat{\Lambda}$ is a well defined, polarized scheme. This is the stable semiabelic pair. Note that the fiber of $X$ over $A$ is a (possibly reducible) projective toric variety, obtained by gluing the toric varieties determined by the tiling in the Delaunay decomposition.

\section{Prym varieties and admissible covers}

It is well known that $\mathcal{M}_{g}$ has a normal crossing compactification $\overline{\mathcal{M}}_{g}$ obtained by allowing stable curves, whose limiting Hodge-theoretic behavior is controlled by a combinatorial object, the dual graph $\Gamma$. Prym varieties are abelian varieties obtained from connected 
étale double covers of curves. A normal crossing compactification for the moduli $\mathcal{R}_{g}$ of connected étale double covers (compatible with $\overline{\mathcal{M}}_{g}$ ) was constructed by Beauville by considering admissible double covers of stable curves. The associated combinatorial object governing the limiting Hodge-theoretic behavior is a dual graph with involution. We briefly review this below.

\subsection{Admissible covers}

Let $C$ be a stable curve of genus $g+1 \geq 2$. Recall that an admissible double cover of $C$ is a finite surjective morphism $\pi: \widetilde{C} \rightarrow C$ of stable curves such that:

(1) The arithmetic genus of $\widetilde{C}$ is equal to $\tilde{g}=2 g+1$.

(2) For each irreducible component $C^{\prime}$ of $C$, the restriction $\pi: \pi^{-1}\left(C^{\prime}\right) \rightarrow C^{\prime}$ has degree two (but $\pi^{-1}\left(C^{\prime}\right)$ may be reducible or disconnected).

(3) If $\iota: \widetilde{C} \rightarrow \widetilde{C}$ is the sheet interchange involution associated to $\pi$, the fixed points of $\iota$ are a subset of the nodes of $\widetilde{C}$, and at a fixed node the local branches of $\widetilde{C}$ are not exchanged.

There exists a smooth, irreducible, proper Deligne-Mumford $\mathbb{C}$-stack $\overline{\mathcal{R}}_{g+1}$ parameterizing admissible double covers of stable curves of genus $g+1 \geq 2$ [Bea77]. We denote by $\mathcal{R}_{g+1}$ the open substack of connected étale double covers of smooth curves. The forgetful functor $\overline{\mathcal{R}}_{g+1} \rightarrow \overline{\mathcal{M}}_{g+1}$ to the moduli of stable curves defines a degree $2^{2(g+1)}-1$ cover, ramified along an irreducible boundary divisor $\delta_{0}^{\text {ram }} \subset \overline{\mathcal{R}}_{g+1}$ (see §3.4). The full boundary $\delta_{\overline{\mathcal{R}}_{g+1}}$ of $\overline{\mathcal{R}}_{g+1}$ is a simple normal crossing divisor with the property that étale locally at a point $\pi: \widetilde{C} \rightarrow C$, its irreducible components correspond to the nodes of $C$. We discuss the irreducible components of the boundary divisor $\delta_{\overline{\mathcal{R}}_{g+1}}$ in $\S 3.4$ below. We denote by $\bar{R}_{g+1}$ and $R_{g+1}$ the coarse moduli spaces of the respective stacks.

\subsection{Involutions of graphs}

Here we fix our conventions on graphs. A graph $\Gamma$ is a set of vertices $V=V(\Gamma)$ and a set of oriented edges $\vec{E}=\vec{E}(\Gamma)$ together with maps $(\vec{E} \stackrel{s}{\rightrightarrows} V, \vec{E} \stackrel{\tau}{\rightarrow} \vec{E})$, where $\tau$ is a fixed-point free involution, and $s$ and $t$ are maps satisfying $s(\vec{e})=t(\tau(\vec{e}))$ for all $\vec{e} \in \vec{E}$. The maps $s$ and $t$ are called the source and target maps respectively.

We define the set of (unoriented) edges to be $E(\Gamma)=E:=\vec{E} / \tau$. Given an oriented edge $\vec{e} \in \vec{E}$ we will denote by $\underline{\vec{e}}$ the class of $\vec{e}$ in $E$. An orientation of an edge $e \in E$ is a representative for $e$ in $\vec{E}$; we use the notation $\vec{e}$ and $\overleftarrow{e}$ for the two possible orientations of $e$. An orientation of a graph $\Gamma$ is a section $\phi: E \rightarrow \vec{E}$ of the quotient map. An oriented graph consists of a pair $(\Gamma, \phi)$ where $\Gamma$ is a graph and $\phi$ is an orientation.

A morphism of graphs $\Gamma_{1} \rightarrow \Gamma_{2}$ consists of a pair of maps

$$
V\left(\Gamma_{1}\right) \rightarrow V\left(\Gamma_{2}\right) \quad \text { and } \quad \vec{E}\left(\Gamma_{1}\right) \rightarrow \vec{E}\left(\Gamma_{2}\right)
$$

such that all of the associated diagrams commute. An involution ı of a graph is an endomorphism of the graph such that $\iota^{2}$ is the identity. We can define morphisms of oriented graphs as well; an involution of an oriented graph is defined in the obvious way. 
Associated to a graph $\Gamma$ is a chain complex

$$
\left(C_{\bullet}(\Gamma, \mathbb{Z}), \partial_{\bullet}\right),
$$

where $C_{0}(\Gamma, \mathbb{Z})$ is the free $\mathbb{Z}$-module with basis $V(\Gamma)$, and $C_{1}(\Gamma, \mathbb{Z})$ is the quotient of the free $\mathbb{Z}$-module with basis $\vec{E}(\Gamma)$ by the relation $\overleftarrow{e}=-\vec{e}$ for every $e \in E(\Gamma)$. We denote by $[\vec{e}]$ the class of $\vec{e}$ in $C_{1}(\Gamma, \mathbb{Z})$. Note that while $\underline{\vec{e}}=\underline{\underline{e}}$ in $E$ (they correspond to the same unoriented edge), in $C_{1}(\Gamma, \mathbb{Z})$ we have $[\vec{e}]=-[\stackrel{e}{e}]$. An orientation $\phi$ determines a basis $\{[\phi(e)]\}_{e \in E}$ for $C_{1}(\Gamma, \mathbb{Z})$, which identifies $C_{1}(\Gamma, \mathbb{Z})$ with the usual chain group of 1-chains for the associated simplicial complex. The boundary map is defined by

$$
\partial: C_{1}(\Gamma, \mathbb{Z}) \rightarrow C_{0}(\Gamma, \mathbb{Z}), \quad[\vec{e}] \mapsto t(\vec{e})-s(\vec{e}) .
$$

We will denote by $H_{\bullet}(\Gamma, \mathbb{Z})$ the homology groups obtained from $C_{\bullet}(\Gamma, \mathbb{Z})$; the group $H_{\bullet}(\Gamma, \mathbb{Z})$ is isomorphic to the homology of the underlying topological space of $\Gamma$.

From the definitions one can check immediately that an involution $\iota$ of a graph $\widetilde{\Gamma}$ induces an involution $\iota$ of the chain complex $C_{\bullet}(\widetilde{\Gamma}, \mathbb{Z})$. This in turn induces an involution $\iota$ on $H_{\bullet}(\widetilde{\Gamma}, \mathbb{Z})$; we denote by $H_{1}(\widetilde{\Gamma}, \mathbb{Z})^{ \pm}$the eigenspaces of the action of $\iota$. We define

$$
H_{1}(\widetilde{\Gamma}, \mathbb{Z})^{[+]}=H_{1}(\widetilde{\Gamma}, \mathbb{Z}) / H_{1}(\widetilde{\Gamma}, \mathbb{Z})^{-} \quad \text { and } \quad H_{1}(\widetilde{\Gamma}, \mathbb{Z})^{[-]}=H_{1}(\widetilde{\Gamma}, \mathbb{Z}) / H_{1}(\widetilde{\Gamma}, \mathbb{Z})^{+} \text {. }
$$

Note that

$$
\begin{aligned}
& H_{1}(\widetilde{\Gamma}, \mathbb{Z})^{[-]} \cong \operatorname{Im}\left(\frac{1}{2}(\operatorname{Id}-\iota)\right) \subseteq \frac{1}{2} H_{1}(\widetilde{\Gamma}, \mathbb{Z}), \\
& H_{1}(\widetilde{\Gamma}, \mathbb{Z})^{[+]} \cong \operatorname{Im}\left(\frac{1}{2}(\operatorname{Id}+\iota)\right) \subseteq \frac{1}{2} H_{1}(\widetilde{\Gamma}, \mathbb{Z}) .
\end{aligned}
$$

As usual, we construct a cochain complex $C^{\bullet}(\widetilde{\Gamma}, \mathbb{Z})=\operatorname{Hom}\left(C_{\bullet}(\widetilde{\Gamma}, \mathbb{Z}), \mathbb{Z}\right)$, and define the cohomology groups $H^{\bullet}(\widetilde{\Gamma}, \mathbb{Z})$ to be the homology of this complex. We have $H^{i}(\widetilde{\Gamma}, \mathbb{Z})=$ $H_{i}(\widetilde{\Gamma}, \mathbb{Z})^{\vee}(i=0,1)$. However, in contrast we have

$$
H^{i}(\widetilde{\Gamma}, \mathbb{Z})^{ \pm}=\left(H_{i}(\widetilde{\Gamma}, \mathbb{Z})^{[ \pm]}\right)^{\vee} \quad(i=0,1) .
$$

Remark 3.1. Here we make an observation that will be useful for later computations. To simplify the discussion, fix an orientation of the graph $\Gamma$. Then, by definition, $C^{1}(\Gamma, \mathbb{Z})=$ $C_{1}(\Gamma, \mathbb{Z})^{\vee}=\left(\bigoplus_{e \in E} \mathbb{Z} \cdot e\right)^{\vee}=\bigoplus_{e \in E} \mathbb{Z} \cdot e^{\vee}$. We call the elements $e^{\vee}$ coedges. There is by definition a surjection $C^{1}(\Gamma, \mathbb{Z}) \rightarrow H^{1}(\Gamma, \mathbb{Z})$. Denote temporarily by $\widehat{e^{\vee}}$ the image of a coedge $e^{\vee}$ in $H^{1}(\Gamma, \mathbb{Z})$. Now note that if $\widehat{e_{1}^{V}}=\widehat{e_{2}^{V}}$, then for any $z \in H_{1}(\Gamma, \mathbb{Z}) \subseteq C_{1}(\Gamma, \mathbb{Z})$, we have $e_{1}^{\vee}(z)=e_{2}^{\vee}(z)$.

An admissible involution of a graph $\widetilde{\Gamma}$ is an involution $\iota$ such that $\iota(\vec{e}) \neq \overleftarrow{e}$ for all $\vec{e} \in \vec{E}$. In other words, an involution is admissible if whenever an unoriented edge of the graph is fixed by the involution, the vertices at the endpoints of the edge are not interchanged by the involution (unless the edge is a loop, in which case the condition requires the associated oriented edges not to be interchanged).

If $\pi: \widetilde{C} \rightarrow C$ is an admissible cover, then the associated involution $\iota$ on $\widetilde{C}$ induces a well defined involution $\iota$ on the vertices $V\left(\Gamma_{\widetilde{C}}\right)$ and on the unoriented edges $E\left(\Gamma_{\widetilde{C}}\right)$. There is also a well defined induced involution on the set of oriented edges. We will call this the induced admissible involution of the dual graph of $\widetilde{C}$. 


\subsection{Prym varieties}

Let $C$ be a stable curve of genus $g \geq 2$. The Jacobian $J C$ is defined to be the connected component of the identity in $\operatorname{Pic}(C)$. The Jacobian is a semiabelian variety of dimension $g$, which can be described explicitly as follows. Let $v: N \rightarrow C$ be the normalization and let $\Gamma=\Gamma_{C}$ be the dual graph of $C$. Then there is an exact sequence

$$
0 \rightarrow H^{1}(\Gamma, \mathbb{Z}) \otimes_{\mathbb{Z}} \mathbb{C}^{*} \rightarrow J C \stackrel{\nu^{*}}{\rightarrow} J N \rightarrow 0 .
$$

The extension determines a class in

$$
\operatorname{Ext}^{1}\left(J N, H^{1}(\Gamma, \mathbb{Z}) \otimes_{\mathbb{Z}} \mathbb{C}^{*}\right)=\operatorname{Hom}\left(H_{1}(\Gamma, \mathbb{Z}), \widehat{J N}\right),
$$

where $\widehat{J N}=\operatorname{Pic}^{0}(J N)$ is the dual abelian variety. We refer the reader to [ABH02, p.76] for an explicit description of the extension class (see also §9). For later reference we note that $J C$ is an extension of the torus $\mathbb{T}_{C}:=H^{1}(\Gamma, \mathbb{Z}) \otimes_{\mathbb{Z}} \mathbb{C}^{*}$, which has character lattice

$$
\Lambda_{C}:=\operatorname{Hom}\left(\mathbb{T}_{C}, \mathbb{C}^{*}\right)=H_{1}(\Gamma, \mathbb{Z}) .
$$

Now let $\pi: \widetilde{C} \rightarrow C$ be an admissible double cover of a stable curve $C$ of genus $g+1 \geq 2$. We define the Prym variety

$$
P:=P(\widetilde{C} / C)=\operatorname{ker}(\mathrm{Nm}: J \widetilde{C} \rightarrow J C)_{0}
$$

to be the connected component of the identity in the kernel of the norm map. The Prym variety is a semiabelian variety of dimension $g$, which can be explicitly described as follows. Let $\tilde{v}: \widetilde{N} \rightarrow \widetilde{C}$ and $\mathcal{v}: N \rightarrow C$ be the normalizations, and let $\tilde{\Gamma}=\Gamma \widetilde{C}$ and $\Gamma=\Gamma_{C}$ be the dual graphs of $\widetilde{C}$ and $C$ respectively. Then there is an exact sequence

$$
0 \rightarrow H^{1}(\widetilde{\Gamma}, \mathbb{Z})^{-} \otimes_{\mathbb{Z}} \mathbb{C}^{*} \rightarrow P \rightarrow A \rightarrow 0,
$$

where $A$ is a finite cover of $P_{N}:=P(\tilde{N} / N)=\operatorname{ker}(\mathrm{Nm}: J \tilde{N} \rightarrow J N)_{0}$, the Prym variety of the normalization. The extension determines a class in

$$
\operatorname{Ext}^{1}\left(A, H^{1}(\widetilde{\Gamma}, \mathbb{Z})^{-} \otimes_{\mathbb{Z}} \mathbb{C}^{*}\right)=\operatorname{Hom}\left(H_{1}(\Gamma, \mathbb{Z})^{[-]}, \widehat{A}\right) .
$$

We direct the reader to $[\mathrm{ABH} 02, \S 1$, Prop. 1.5] for more details on the relationship between $A$ and $P_{N}$, as well as for an explicit description of the extension class (see also $§ 9$ ). For later reference we note that $P$ is an extension of the torus $\mathbb{T}_{P}:=H^{1}(\widetilde{\Gamma}, \mathbb{Z})^{-} \otimes_{\mathbb{Z}} \mathbb{C}^{*}$, which has character lattice

$$
\Lambda_{\widetilde{C} / C}:=\operatorname{Hom}\left(\mathbb{T}_{P}, \mathbb{C}^{*}\right)=H_{1}(\Gamma, \mathbb{Z})^{[-]} .
$$

\subsection{The boundary divisors in $\overline{\mathcal{R}}_{g+1}$}

In some arguments in what follows we will want to enumerate certain types of admissible covers. We thus review the enumeration of the irreducible boundary components of $\overline{\mathcal{R}}_{g+1}$ following [Far12] and the references therein (see especially [Ber99], [FL10]), noting also the corresponding descriptions in terms of vanishing cycles (see also the preprint version of [FS86]). 
Recall that for a smooth curve $C$ of genus $g$, there are natural identifications of the following sets:

$$
\begin{aligned}
& \{\text { Conn. ét. dbl. cov. } \pi: \widetilde{C} \rightarrow C\}=H^{1}(C, \mathbb{Z} / 2 \mathbb{Z}) \backslash\{0\} \\
& =\left\{\eta \in \operatorname{Pic}^{0}(C): \eta \not \mathscr{O}_{C}, \eta^{\otimes 2} \cong \mathscr{O}_{C}\right\} .
\end{aligned}
$$

For a stable curve $C_{0}$, with a unique node, we will denote by $C_{*}$ a nearby smooth curve, and $\gamma \in H^{1}\left(C_{*}, \mathbb{Z}\right)$ the associated vanishing cocycle. The irreducible boundary components of $\overline{\mathcal{R}}_{g+1}$ are as follows. The preimage in $\overline{\mathcal{R}}_{g+1}$ of the locus of irreducible stable curves $\delta_{0} \subset \overline{\mathcal{M}}_{g+1}$ has three irreducible components $\delta_{0}^{\prime}$, $\delta_{0}^{\prime \prime}$, and $\delta_{0}^{\text {ram }}$ defined as follows:

$$
\begin{aligned}
\delta_{0}^{\prime} & =\left\{\left(C_{0}, a\right): C_{0} \in \delta_{0}^{\circ}, a \in H^{1}\left(C_{*}, \mathbb{Z} / 2 \mathbb{Z}\right) \backslash\{0\}, a \cdot \gamma=0, \text { but } a \notin\langle\gamma\rangle\right\}^{-}, \\
\delta_{0}^{\prime \prime} & =\left\{\left(C_{0}, a\right): C_{0} \in \delta_{0}^{\circ}, a \in H^{1}\left(C_{*}, \mathbb{Z} / 2 \mathbb{Z}\right) \backslash\{0\}, a \cdot \gamma=0, \text { and } a \in\langle\gamma\rangle\right\}^{-}, \\
\delta_{0}^{\mathrm{ram}} & =\left\{\left(C_{0}, a\right): C_{0} \in \delta_{0}^{\circ}, a \in H^{1}\left(C_{*}, \mathbb{Z} / 2 \mathbb{Z}\right) \backslash\{0\}, a \cdot \gamma \neq 0\right\}^{-} .
\end{aligned}
$$

In the above, and in what follows, we will denote by $\delta_{i}^{\circ} \subseteq \delta_{i}$ the locus of curves with a single node. The bar after the sets above denotes taking the closure.
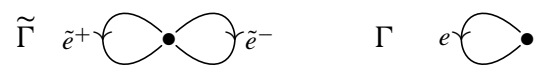

Fig. 1. Dual graph of a generic admissible cover in $\delta_{0}^{\prime}$.

$\widetilde{\Gamma}$
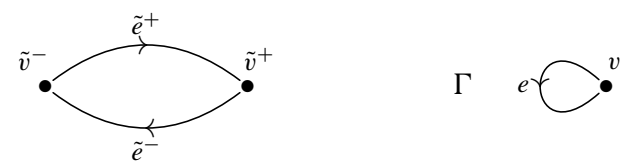

Fig. 2. Dual graph of a generic admissible cover in $\delta_{0}^{\prime \prime}$.

$\widetilde{\Gamma}$

Fig. 3. Dual graph of a generic admissible cover in $\delta_{0}^{\text {ram }}$.

The preimage in $\overline{\mathcal{R}}_{g+1}$ of the boundary divisor $\delta_{i} \subset \overline{\mathcal{M}}_{g+1}$ has three irreducible components (only two for $i=(g+1) / 2$, in which case the first two are the same):

$$
\begin{aligned}
\delta_{i} & =\left\{(C, \eta): C=C_{i} \cup C_{g+1-i} \in \delta_{i}^{\circ},\left.\eta\right|_{C_{g+1-i}} \cong \mathscr{O}_{C_{g+1-i}}\right\}^{-}, \\
\delta_{g+1-i} & =\left\{(C, \eta): C=C_{i} \cup C_{g+1-i} \in \delta_{i}^{\circ},\left.\eta\right|_{C_{i}} \cong \mathscr{O}_{C_{i}}\right\}^{-}, \\
\delta_{i, g+1-i} & =\left\{(C, \eta): C=C_{i} \cup C_{g+1-i} \in \delta_{i}^{\circ},\left.\eta\right|_{C_{i}} ¥ \mathscr{O}_{C_{i}},\left.\eta\right|_{C_{g+1-i}} ¥ \mathscr{O}_{C_{g+1-i}}\right\}^{-} .
\end{aligned}
$$




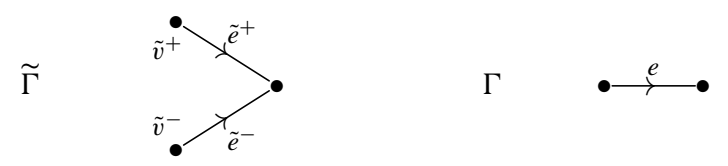

Fig. 4. Dual graph of a generic admissible cover in $\delta_{i}\left(\right.$ or $\left.\delta_{g+1-i}\right)$.

$\widetilde{\Gamma}$

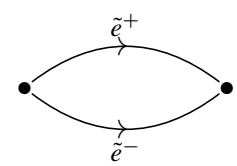

$\Gamma$

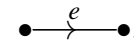

Fig. 5. Dual graph of a generic admissible cover in $\delta_{i, g+1-i}$

\section{Monodromy cones for Prym varieties}

In this section, we compute the monodromy cones (in the terminology of $\S 2$ ) for a boundary point $(\widetilde{C}, C)$ of $\bar{R}_{g+1}$ in terms of the combinatorics of the dual graph of $(\widetilde{C}, C)$ (discussed in $\S 3$ ). As a warm-up, we first review the classical case of Jacobians. The case of Pryms then naturally follows. While essentially equivalent computations can be found in [FS86] and [ABH02], our presentation for Prym varieties seems to be somewhat new.

\subsection{Monodromy cones for stable curves}

Let $C$ be a stable curve of genus $g \geq 2$. Let $\mathscr{C} \rightarrow B$ be a miniversal deformation of $C$, with discriminant $\Delta$, and set $B^{\circ}=B_{C}^{\circ}=B-\Delta$. Denote by $0 \in B$ the point corresponding to $C$. Let $\Gamma$ be the dual graph of $C$. We have $\operatorname{dim} B_{C}=3 g-3$ and $\Delta$ is a collection of simple normal crossing hyperplanes, indexed by the nodes of $C$ (which in turn are indexed by the edges of $\Gamma$ ). Recall that the Jacobian of $C$ is a semiabelian variety obtained as an extension of the torus $\mathbb{T}_{C}=H^{1}(\Gamma, \mathbb{Z}) \otimes_{\mathbb{Z}} \mathbb{C}^{*}$, which has character lattice

$$
\Lambda_{C}=H_{1}(\Gamma, \mathbb{Z}) .
$$

Since $B^{\circ}$ is locally (near 0 ) a polycylinder and the associated monodromies are unipotent, we can apply the considerations of $\S 2.1 .2$ and $\S 2.2$ and define the monodromy cone

$$
\sigma(C) \subseteq \bar{C}^{\mathbb{Q}}\left(\Lambda_{C}\right)
$$

to be the cone spanned by the log of monodromies around the branches of $\Delta$ (see (2.4)). More precisely, we recall that for each irreducible component $\Delta_{e} \subseteq \Delta$, corresponding to an edge $e \in \Gamma$, there is an associated quadratic form obtained from the log of monodromy around $\Delta_{e}$ (cf. (2.11)). The closure $\bar{\sigma}(C)$ is the cone generated by these quadratic forms $(\operatorname{see}(2.15))$ :

$$
\bar{\sigma}(C)=\mathbb{R}_{\geq 0}\left\langle\bar{N}_{e}\right\rangle_{e \in \Gamma} \subseteq \operatorname{Hom}\left(\operatorname{Sym}^{2} H_{1}(\Gamma, \mathbb{Q}), \mathbb{Q}\right)_{\mathbb{R}} .
$$

We now state the following well known description of the monodromy cone, and provide a sketch of the proof. 
Proposition 4.1. Suppose that $C$ is a stable curve of genus $g \geq 2$. Let $e$ be an edge of the dual graph $\Gamma$ of $C$. Then $\left(e^{\vee}\right)^{2}$ is the quadratic form obtained as the log of monodromy around the corresponding component $\Delta_{e}$ of the discriminant. Consequently, the closure of the monodromy cone for $C$ is

$$
\bar{\sigma}(C)=\mathbb{R}_{\geq 0}\left\langle\left(e^{\vee}\right)^{2}\right\rangle_{e \in E(\Gamma)} .
$$

Proof. We start by describing the monodromy operators as in (2.11). Let us first consider the special case where $C$ has a single node. There are two possibilities:

(0) $e^{\vee}=0 \in H^{1}(\Gamma, \mathbb{Q})$ (equivalently, $C \in \delta_{i}, i>0$ ).

(1) $e^{\vee} \neq 0 \in H^{1}(\Gamma, \mathbb{Q})$ (equivalently, $C \in \delta_{0}$ ).

In case $(0), H_{1}(\Gamma, \mathbb{Q})=0$, so $J C$ is an abelian variety, the monodromy is trivial, and there is nothing to show. In case $(1), H_{1}(\Gamma, \mathbb{Q})=\mathbb{Q}\langle e\rangle$, where $e$ is the unique edge of $\Gamma$. As before, we view the log of monodromy as a map

$$
H_{1}(\Gamma, \mathbb{Q}) \stackrel{N_{e}}{\longrightarrow} H^{1}(\Gamma, \mathbb{Q})=\left(H_{1}(\Gamma, \mathbb{Q})\right)^{\vee} .
$$

Since the monodromy operator $T_{e}$ is given by the well known Picard-Lefschetz transformation, it follows that $N_{e}(e)=e^{\vee}$. The associated quadratic form is then $\left(e^{\vee}\right)^{2}$.

The general case follows by the arguments in $\S 1$ and $\S 2$ (especially $\S 2.2$ and (2.14)), which establish that $\bar{N}_{e}$ is given by the composition

$$
H_{1}(\Gamma, \mathbb{Q}) \rightarrow H_{1}\left(\Gamma_{e}, \mathbb{Q}\right) \stackrel{N_{e}}{\longrightarrow} H^{1}\left(\Gamma_{e}, \mathbb{Q}\right) \rightarrow H^{1}(\Gamma, \mathbb{Q})
$$

where $\Gamma_{e}$ is the dual graph of the curve obtained from $C$ by smoothing all of the nodes except for the one corresponding to $e$.

Remark 4.2. Let $\psi: S \rightarrow B$ be a morphism from the unit disk to a miniversal deformation space of the curve $C$, induced from a 1-parameter deformation of $C$. For each edge $e$ of $\Gamma$, the dual graph of $C$, let $z_{e}$ be a local parameter defining the hyperplane $H_{e} \subseteq B$ parameterizing curves with all nodes smoothed except the one corresponding to $e$. Then the log of monodromy for the family is given by $\sum_{e \in \Gamma}$ ord $\psi^{*}\left(z_{e}\right)\left(e^{\vee}\right)^{2}$.

\subsection{Monodromy cones for admissible double covers}

Let $\pi: \widetilde{C} \rightarrow C$ be an admissible cover of a stable curve $C$ of arithmetic genus $g+1 \geq 2$. Let $B$ be the base of a miniversal deformation of the cover, with discriminant $\Delta$, and set $B^{\circ}=B-\Delta$. Denote by $0 \in B$ the point corresponding to $\pi: \widetilde{C} \rightarrow C$. Let $\widetilde{\Gamma}(\operatorname{resp} . \Gamma)$ be the dual graph of $\widetilde{C}$ (resp. $C$ ). We have $\operatorname{dim} B=3 g$ and $\Delta$ is a collection of simple normal crossing hyperplanes, indexed by the nodes of $C$ (which are in turn indexed by the edges of $\Gamma$ ). Recall that the Prym variety of $\pi: \widetilde{C} \rightarrow C$ is a semiabelian variety obtained as an extension of the torus $\mathbb{T}_{P}=H^{1}(\widetilde{\Gamma}, \mathbb{Z})^{-} \otimes_{\mathbb{Z}} \mathbb{C}^{*}$, which has character lattice

$$
\Lambda_{\widetilde{C} / C}=H_{1}(\Gamma, \mathbb{Z})^{[-]} .
$$


We wish to describe the associated (log of) monodromy cone

$$
\sigma(\widetilde{C} / C) \subseteq \bar{C}^{\mathbb{Q}}\left(\Lambda_{\widetilde{C} / C}\right)
$$

Recall that for each irreducible component $\Delta_{e} \subseteq \Delta$, corresponding to an edge $e \in \Gamma$, there is an associated quadratic form obtained as the log of monodromy around $\Delta_{e}$. The closure $\bar{\sigma}(\widetilde{C} / C)$ is the cone generated by these quadratic forms.

From the perspective of Hodge theory, for each 1-parameter family $f: S \rightarrow B$ with $f(0)=0 \in B$, there is an associated 1-parameter variation of Hodge structures determined by the family of Prym varieties. We will denote by $N^{-}$the $\log$ of monodromy for this VHS. At the same time, there is a 1-parameter VHS determined by the Jacobians of the covering curves, with log of monodromy $\tilde{N}$. One can identify $\operatorname{Gr}_{0}\left(N^{-}\right)=$ $\left(\operatorname{Gr}_{0} \widetilde{N}\right)^{-}=H^{1}(\widetilde{\Gamma}, \mathbb{Q})^{-}$. Then as in $(2.3)$, we can view the log of monodromy as a map

$$
H_{1}(\widetilde{\Gamma}, \mathbb{Q})^{-} \stackrel{N^{-}}{\longrightarrow} H^{1}(\widetilde{\Gamma}, \mathbb{Q})^{-}
$$

or dually as a positive definite quadratic form on $H_{1}(\widetilde{\Gamma}, \mathbb{Q})^{-}$. Now to describe the closure of the monodromy cone, for each edge $e \in \Gamma$, one obtains a log of monodromy operator

$$
H_{1}(\widetilde{\Gamma}, \mathbb{Q})^{-} \stackrel{\bar{N}_{e}^{-}}{\longrightarrow} H^{1}(\widetilde{\Gamma}, \mathbb{Q})^{-},
$$

so that the associated bilinear form is symmetric and positive semidefinite. The closure of the monodromy cone is then given as

$$
\bar{\sigma}(C)=\mathbb{R}_{\geq 0}\left\langle\bar{N}_{e}^{-}\right\rangle_{e \in \Gamma} \subseteq \operatorname{Hom}\left(\operatorname{Sym}^{2} H_{1}(\widetilde{\Gamma}, \mathbb{Q})^{-}, \mathbb{Q}\right)_{\mathbb{R}}
$$

Proposition 4.3. Suppose that $\pi: \widetilde{C} \rightarrow C$ is an admissible cover of a stable curve of genus $g+1 \geq 2$. Let $e$ be an edge of the dual graph $\Gamma$ of $C$ and let $\tilde{e}$ be an edge of $\tilde{\Gamma}$ lying above $e$. Then (up to a scalar factor) $\left(\tilde{e}^{\vee}-i \tilde{e}^{\vee}\right)^{2}$ is the quadratic form obtained as the log of monodromy around the corresponding component $\Delta_{e}$ of the discriminant. Consequently, the closure of the monodromy cone is

$$
\bar{\sigma}(\tilde{C} / C)=\mathbb{R}_{\geq 0}\left\langle\left(\tilde{e}^{\vee}-\iota \tilde{e}^{\vee}\right)^{2}\right\rangle_{e \in E(\Gamma)} .
$$

Proof. Let $\pi: \widetilde{C} \rightarrow C$ be an admissible cover of a stable curve of genus $g+1 \geq 2$. We will start by describing the closure of the monodromy cone via the log of monodromy as described in (4.2).

First we will consider the special case where $C$ has a unique node. It is convenient to break this down further into three subcases. To do this, let us fix some notation. Let $e$ be the unique edge of $\Gamma$, the dual graph of $C$. Let $\tilde{e}$ be an edge of $\widetilde{\Gamma}$ lying over $e$. Then exactly one of the following holds:

(0) $\tilde{e}^{\vee}-\iota \tilde{e}^{\vee}=0 \in H^{1}(\widetilde{\Gamma}, \mathbb{Q})$ (equivalently, $\widetilde{C} \rightarrow C \in \delta_{\overline{\mathcal{R}}_{g+1}} \backslash\left(\delta_{i, g+1-i} \cup \delta_{0}^{\prime}\right)$ ).

(1) $\tilde{e}^{\vee}-i \tilde{e}^{\vee}=2 \tilde{e}^{\vee} \neq 0 \in H^{1}(\widetilde{\Gamma}, \mathbb{Q})$ (equivalently, $\widetilde{C} \rightarrow C \in \delta_{i, g+1-i}$ ).

(2) $\tilde{e}^{\vee}-i \tilde{e}^{\vee} \neq 2 \tilde{e}^{\vee}, 0 \in H^{1}(\widetilde{\Gamma}, \mathbb{Q})$ (equivalently, $\widetilde{C} \rightarrow C \in \delta_{0}^{\prime}$ ). 
In case $(0), H^{1}(\widetilde{\Gamma}, \mathbb{Q})^{-}=0$, the Prym is an abelian variety, the monodromy is trivial, and there is nothing to show. In cases $(1)$ and $(2), H_{1}(\widetilde{\Gamma}, \mathbb{Q})^{-}=\mathbb{Q}\langle\tilde{e}-i \tilde{e}\rangle$, where $e$ is the unique edge of $\Gamma$ and $\tilde{e}$ and $\iota \tilde{e}$ are the edges of $\widetilde{\Gamma}$ over $e$ interchanged by the involution.

Now we will describe the $\log$ of monodromy as a map

$$
H_{1}(\widetilde{\Gamma}, \mathbb{Q})^{-} \stackrel{N_{e}^{-}}{\longrightarrow} H^{1}(\widetilde{\Gamma}, \mathbb{Q})^{-}=\left(H_{1}(\widetilde{\Gamma}, \mathbb{Q})^{-}\right)^{\vee}
$$

To do this, let us fix an appropriate base point $* \in B$ with $\widetilde{C}_{*} \rightarrow C_{*}$ an étale double cover. In suitable coordinates on $H^{1}\left(\widetilde{C}_{*}, \mathbb{C}\right)^{-}$, the monodromy operator is given by

$$
T_{e}=\left(\begin{array}{ccccc}
1 & 2 & 0 & \cdots & 0 \\
0 & 1 & 0 & \cdots & 0 \\
\vdots & & \ddots & & \vdots \\
0 & \cdots & 0 & 1 & 0 \\
0 & \cdots & 0 & 0 & 1
\end{array}\right) \quad\left(\text { resp. } \quad T_{e}=\left(\begin{array}{ccccc}
1 & 1 & 0 & \cdots & 0 \\
0 & 1 & 0 & \cdots & 0 \\
\vdots & & \ddots & & \vdots \\
0 & \cdots & 0 & 1 & 0 \\
0 & \cdots & 0 & 0 & 1
\end{array}\right)\right) .
$$

This follows from the classification of admissible covers in $\$ 3.4$ (see also [FS86]). Since the $\log$ of monodromy is given by $N_{e}=T_{e}-\mathrm{Id}$, it follows that (up to a scalar multiple) $N_{e}^{-}(\tilde{e}-i \tilde{e})=\tilde{e}^{\vee}-i \tilde{e}^{\vee}$. Thus the associated quadratic form is $\left(\tilde{e}^{\vee}-i \tilde{e}^{\vee}\right)^{2}$.

The general case then follows by the arguments in $\S 1, \S 2$ (esp. $\$ 2.2$ and (2.14)) by considering the composition

$$
H_{1}(\widetilde{\Gamma}, \mathbb{Q})^{-} \rightarrow H_{1}\left(\widetilde{\Gamma}_{e}, \mathbb{Q}\right)^{-} \stackrel{N_{e}^{-}}{\longrightarrow} H^{1}\left(\widetilde{\Gamma}_{e}, \mathbb{Q}\right)^{-} \rightarrow H^{1}(\widetilde{\Gamma}, \mathbb{Q})^{-},
$$

where $\widetilde{\Gamma}_{e}$ is the dual graph of the curve obtained from $\widetilde{C}$ by smoothing all of the nodes except those lying above the node of $C$ corresponding to $e$.

Remark 4.4. Let $\psi: S \rightarrow B$ be a morphism from the disk to a miniversal deformation space of the admissible cover $\widetilde{C} \rightarrow C$ induced from a 1-parameter deformation of $\widetilde{C} \rightarrow C$. For each edge $e \in \Gamma$, the dual graph of $C$, let $z_{e}$ be a local parameter defining the hyperplane $\Delta_{e} \subseteq B$ parameterizing covers with all nodes smoothed except those corresponding to $e$. Then the log of monodromy for the family is given by $\sum_{e \in \Gamma} \operatorname{ord} \psi^{*}\left(z_{e}\right)\left(\tilde{e}^{\vee}-i \tilde{e}^{\vee}\right)^{2}$.

\section{Extension criteria for the Torelli and Prym map}

After the preliminaries of the previous sections, we can state our results regarding the extension of the period maps to various toroidal compactifications. In general given a period map $\mathcal{M} \rightarrow \mathcal{A}_{g}$ and a normal crossing compactification $\mathcal{M} \subset \overline{\mathcal{M}}$, the question of extending to the boundary essentially boils down to two steps: the computation of monodromy cones (which we did in the previous section) and then a check that a monodromy cone is contained in one of the cones of the fan defining a toroidal compactification (a combinatorial statement). Of course, this general process is well known and occurs in various guises in the literature, but its systematic application in the case of admissible covers gives a good and uniform understanding of the extensions of Prym maps to toroidal compactifications. 


\subsection{Extension of the Torelli map}

To motivate the arguments for the Prym map, we first review the results of Alexeev and Brunyate [AB12] for the Torelli map. Throughout this subsection, we will use the following notation. Fix $g \geq 2$ and a stable curve $C$ in $\overline{\mathcal{M}}_{g}$. Let $\Gamma$ be the dual graph. Recall from Proposition 4.1 that the closure of the monodromy cone for the admissible cover is the cone

$$
\bar{\sigma}(C):=\mathbb{R}_{\geq 0}\left\langle\left(e^{\vee}\right)^{2}\right\rangle_{e \in E(\Gamma)} \subseteq\left(\operatorname{Sym}^{2} H_{1}(\Gamma, \mathbb{Z})\right)_{\mathbb{R}}^{\vee} .
$$

Fix a free $\mathbb{Z}$-module $\Lambda$ of rank $g$, and a $\operatorname{GL}(\Lambda)$-admissible cone decomposition $\Sigma$ of $\bar{C}^{\mathbb{Q}}(\Lambda)$. Let $\bar{A}_{g}^{\Sigma}$ be the associated toroidal compactification. Fix a surjection $\Lambda \rightarrow \Lambda_{C}=H_{1}(\Gamma, \mathbb{Z})$, and denote by $\Sigma_{C}$ the $\mathrm{GL}\left(\Lambda_{C}\right)$-admissible cone decomposition of $\bar{C}^{\mathbb{Q}}\left(\Lambda_{C}\right)$ (induced by the inclusion $\bar{C}^{\mathbb{Q}}\left(\Lambda_{C}\right) \hookrightarrow \bar{C}^{\mathbb{Q}}(\Lambda)$ ). Recall that $\Sigma_{C}$ does not depend on the surjection $\Lambda \rightarrow \Lambda_{C}$. We now collect results from the literature due to Mumford and Namikawa [Nam76] and Alexeev and Brunyate [AB12].

Theorem 5.1. Fix $g \geq 2$. The Torelli map

$$
J^{\Sigma}: \bar{M}_{g} \rightarrow \bar{A}_{g}^{\Sigma}
$$

extends to a morphism in a neighborhood of a stable curve $C$ if and only if there exists a cone $\sigma \in \Sigma_{C}$ of the admissible decomposition containing the monodromy cone $\sigma(C)$.

(1) (Mumford-Namikawa [Nam76, Cor. 18.9]) The Torelli map extends to a morphism to the second Voronoi compactification:

$$
J^{V}: \bar{M}_{g} \rightarrow \bar{A}_{g}^{V}
$$

(2) (Alexeev-Brunyate $[\mathrm{AB} 12, \mathrm{Thm} .4 .7, \mathrm{Thm} .6 .7])$ The Torelli map extends to a morphism to the perfect cone compactification:

$$
J^{P}: \bar{M}_{g} \rightarrow \bar{A}_{g}^{P} .
$$

(3) (Alexeev-Brunyate $[\mathrm{AB} 12, \mathrm{Thm} .4 .8]$ ) The Torelli map extends to a morphism to $\bar{A}_{g}^{C}$ in a neighborhood of $C \in \bar{M}_{g}$ if and only if there exists a quadratic form $Q$ on $H^{1}(\Gamma, \mathbb{R})$ such that

(a) $Q(r)>0$ for all $r \in H^{1}(\Gamma, \mathbb{R}) \backslash\{0\}$, i.e. $Q$ is positive definite.

(b) $Q(\ell) \geq 1$ for all $\ell \in H^{1}(\Gamma, \mathbb{Z}) \backslash\{0\}$.

(c) $Q\left(e^{\vee}\right)=1$ for all $e \in E(\Gamma)$ such that $e^{\vee} \neq 0$.

(d) $Q(\ell) \in \mathbb{Z}$ for all $\ell \in H^{1}(\Gamma, \mathbb{Z})$.

Proof. The first statement of the theorem follows from the standard results on toroidal compactifications discussed in $\S 2.3$. We sketch the proofs of the remaining parts following Alexeev and Brunyate.

(1) From Proposition 4.3 and Lemma 1.2, it follows that the Torelli map extends to a morphism to $\bar{A}_{g}^{V}$ in a neighborhood of $C$ if and only if for any collection $e_{1}, \ldots, e_{m}$ $\in E(\Gamma)$ of edges such that the cocycles $e_{1}^{\vee}, \ldots, e_{m}^{\vee}$ form a basis for $H^{1}(\Gamma, \mathbb{R})$, these 
cocycles in fact form a $\mathbb{Z}$-basis for $H^{1}(\Gamma, \mathbb{Z})$. On the other hand, an elementary result from graph theory (see e.g. [AB12, Lem. 3.3], [ABH02, (J6), p. 95]) asserts the following: For a graph $\Gamma$ and $e_{1}, \ldots, e_{m} \in E(\Gamma)$, the cocycles $e_{1}^{\vee}, \ldots, e_{m}^{\vee}$ form a $\mathbb{Z}$-basis of $H^{1}(\Gamma, \mathbb{Z})$ if and only if they form an $\mathbb{R}$-basis of $H^{1}(\Gamma, \mathbb{R})$, if and only if the graph obtained from $\Gamma$ by removing the edges $e_{1}, \ldots, e_{m}$ is a spanning tree (i.e. $b_{0}=1, b_{1}=0$, and it contains all the vertices). This completes the proof.

(2) The monodromy cone is generated by rank 1 quadrics, and in the previous paragraph it was shown to be matroidal. Consequently, the monodromy cone is a perfect cone [MV12, Thm. A] (see Remarks 1.6 and 5.2). Thus the period map extends.

(3) This is a restatement of Lemma 1.7.

Remark 5.2. For (2), it should be noted that from Lemma 1.5 it follows that the Torelli map extends in a neighborhood of $C \in \bar{M}_{g}$ if and only if there exists a positive definite quadratic form $Q$ on $H^{1}(\Gamma, \mathbb{R})$ such that $Q(\ell) \geq 1$ for all $\ell \in H^{1}(\Gamma, \mathbb{Z}) \backslash\{0\}$ and $Q\left(e^{\vee}\right)=1$, for all $e \in E(\Gamma)$ such that $e^{\vee} \neq 0$. In [AB12, Thm. 6.7, p. 194] Alexeev and Brunyate establish the existence of such quadratic forms, providing the proof of this case of [MV12, Thm. A].

Remark 5.3. For $g \leq 4$ the central cone compactification agrees with the perfect cone compactification, and consequently the Torelli map extends to a morphism to $\bar{A}_{g}^{C}$ for $g \leq 4$. In fact, in [AB12, Cor. 5.4], [ $\mathrm{AL}^{+} 12$, Cor. 1.2] it is established that all dual graphs of genus $g \leq 8$ admit a quadratic form as in Theorem 5.1(3), and so the Torelli map extends to a morphism to $\bar{A}_{g}^{C}$ for all $g \leq 8$. On the other hand, there are dual graphs of curves of all genera $g \geq 9$ that do not admit such quadratic forms [AB12, Cor. 5.6]. Consequently, the Torelli map does not extend to a morphism to $\bar{A}_{g}^{C}$ for $g \geq 9$.

Remark 5.4. Recall from Remark 2.7 that the Torelli map extends to a toroidal compactification, as a map of stacks, if and only if it extends as a map of the coarse moduli spaces.

\subsection{Extension of the Prym map}

Throughout this subsection, we will use the following notation. Fix $g+1 \geq 2$ and $\pi$ : $\widetilde{C} \rightarrow C$ an admissible double cover in $\overline{\mathcal{R}}_{g+1}$. Let $\widetilde{\Gamma}$ and $\Gamma$ be the dual graphs of $\widetilde{C}$ and $C$, respectively. To simplify the discussion, fix once and for all, for each edge $e \in E(\Gamma)$, a choice of edge $\tilde{e} \in E(\widetilde{\Gamma})$ lying over $e$. Having made this choice, then for each edge $e \in E(\Gamma)$, fix a cocycle $\ell_{e} \in H^{1}(\widetilde{\Gamma}, \mathbb{Z})^{-}$by the rule

$$
\ell_{e}:= \begin{cases}\tilde{e}^{\vee}-i \tilde{e}^{\vee} & \text { if } i \tilde{e}^{\vee} \neq-\tilde{e}^{\vee} \in H^{1}(\widetilde{\Gamma}, \mathbb{Z}), \\ \tilde{e}^{\vee} & \text { if } i \tilde{e}^{\vee}=-\tilde{e}^{\vee} \in H^{1}(\widetilde{\Gamma}, \mathbb{Z}) .\end{cases}
$$

Recall from Proposition 4.3 that the closure of the monodromy cone for the admissible cover is the cone

$$
\bar{\sigma}(\widetilde{C} / C):=\mathbb{R}_{\geq 0}\left\langle\ell_{e}^{2}\right\rangle_{e \in E(\Gamma)} \subseteq\left(\operatorname{Sym}^{2} H_{1}(\widetilde{\Gamma}, \mathbb{Z})^{[-]}\right)_{\mathbb{R}}^{\vee} .
$$

Note that $\ell_{e}^{2}$ does not depend on the choice of $\tilde{e}$ lying over a fixed $e \in \Gamma$. 
Remark 5.5. The definition of $\ell_{e}$ is made to ensure that $\ell_{e}$ is primitive in $H^{1}(\widetilde{\Gamma}, \mathbb{Z})^{-}$. It is important that one takes the condition $\iota \tilde{e}^{\vee}=-\tilde{e}^{\vee}$ as being in $H^{1}(\widetilde{\Gamma}, \mathbb{Z})$. Note in particular that $\iota \tilde{e}^{\vee}$ never agrees with $-\tilde{e}^{\vee}$ in $C^{1}(\widetilde{\Gamma}, \mathbb{Z})$, but always agrees with $-\tilde{e}^{\vee}$ viewed as a linear function on $H_{1}(\widetilde{\Gamma}, \mathbb{Z})^{[-]}$.

Fix a free $\mathbb{Z}$-module $\Lambda$ of rank $g$, and a GL( $\Lambda)$-admissible cone decomposition $\Sigma$ of $\bar{C}^{\mathbb{Q}}(\Lambda)$. Let $\bar{A}_{g}^{\Sigma}$ be the associated toroidal compactification. Fix a surjection $\Lambda \rightarrow$ $\Lambda_{\widetilde{C} / C}=H_{1}(\widetilde{\Gamma}, \mathbb{Z})^{[-]}$, and denote by $\Sigma_{\widetilde{C} / C}$ the $\operatorname{GL}\left(\Lambda_{\widetilde{C} / C}\right)$-admissible cone decomposition of $\bar{C}^{\mathbb{Q}}\left(\Lambda_{\tilde{C} / C}\right)$ (induced by the inclusion $\bar{C}^{\mathbb{Q}}\left(\Lambda_{\tilde{C} / C}\right) \hookrightarrow \bar{C}^{\mathbb{Q}}(\Lambda)$ ). Recall that $\Sigma_{\tilde{C} / C}$ does not depend on the surjection $\Lambda \rightarrow \Lambda_{\widetilde{C} / C}$. We now use this to prove an extension theorem for the Prym map. The case of the second Voronoi compactification gives another proof of [ABH02, Thm. 3.2(1), (4)] (see Remark 5.7 below), while the results for the perfect and central cone are new.

Theorem 5.6. Fix $g \geq 1$. The Prym map

$$
P^{\Sigma}: \bar{R}_{g+1} \rightarrow \bar{A}_{g}^{\Sigma}
$$

extends to a morphism in a neighborhood of an admissible cover $\pi: \widetilde{C} \rightarrow C$ if and only if there exists a cone $\sigma \in \Sigma_{\widetilde{C} / C}$ of the admissible decomposition containing the monodromy cone $\sigma(\widetilde{C} / C)$.

(1) (Alexeev-Birkenhake-Hulek [ABH02, Thm. 3.2(1), (4)]) The Prym map extends to a morphism to the second Voronoi compactification $\bar{A}_{g}^{V}$ in a neighborhood of ( $\pi$ : $\widetilde{C} \rightarrow C) \in \bar{R}_{g+1}$ if and only if:

(V) For any collection $e_{1}, \ldots, e_{m} \in E(\Gamma)$ such that the corresponding cocycles $\ell_{e_{1}}, \ldots, \ell_{e_{m}}$ form a basis for $H^{1}(\widetilde{\Gamma}, \mathbb{R})^{-}$, the cocycles $\ell_{e_{1}}, \ldots, \ell_{e_{m}}$ in fact form a $\mathbb{Z}$-basis for $H^{1}(\widetilde{\Gamma}, \mathbb{Z})^{-}$.

(2) The Prym map extends to a morphism to the perfect cone compactification $\bar{A}_{g}^{P}$ in a neighborhood of $(\pi: \widetilde{C} \rightarrow C) \in \bar{R}_{g+1}$ if and only if:

(P) There exists a quadratic form $Q$ on $H^{1}(\widetilde{\Gamma}, \mathbb{R})^{-}$such that:

(i) $Q(r)>0$ for all $r \in H^{1}(\widetilde{\Gamma}, \mathbb{R})^{-} \backslash\{0\}$, i.e. $Q$ is positive definite.

(ii) $Q(\ell) \geq 1$ for all $\ell \in H^{1}(\widetilde{\Gamma}, \mathbb{Z})^{-} \backslash\{0\}$.

(iii) $Q\left(\ell_{e}\right)=1$ for all $e \in E(\Gamma)$ such that $\ell_{e} \neq 0$.

(3) The Prym map extends to a morphism to the central cone compactification $\bar{A}_{g}^{C}$ in a neighborhood of $(\pi: \widetilde{C} \rightarrow C) \in \bar{R}_{g+1}$ if and only if:

(C) There exists a quadratic form $Q$ on $H^{1}(\widetilde{\Gamma}, \mathbb{R})^{-}$such that in addition to satisfying (i)-(iii) above, $Q$ also satisfies:

(iv) $Q(\ell) \in \mathbb{Z}$ for all $\ell \in H^{1}(\widetilde{\Gamma}, \mathbb{Z})^{-}$.

Proof. The first statement of the theorem follows from the standard results on toroidal compactifications discussed in $\$ 2.3$; (1) then follows from Proposition 4.3 and Lemma 1.2; (2) follows from Lemma 1.5; and (3) from Lemma 1.7. 
Remark 5.7. To see that Theorem 5.6(1) is equivalent to [ABH02, Thm. 3.2(1), (4)] observe that it follows from Lemma 1.3 that $(\mathrm{V})$ is equivalent to

$\left(\mathrm{V}^{\prime}\right)$ The linear functions $\left\{\ell_{e}\right\}_{e \in E(\Gamma)}$ define a dicing of the lattice $H_{1}(\widetilde{\Gamma}, \mathbb{Z})^{[-]}$.

Then note that as functions on $H_{1}(\widetilde{\Gamma}, \mathbb{Z})^{[-]}$, the linear forms $\tilde{e}^{\vee}-i \tilde{e}^{\vee}$ and $2 \tilde{e}^{\vee}$ agree (see Remark 5.5). Thus condition $\left(\mathrm{V}^{\prime}\right)$ here is the same as condition (*) of [ABH02, p. 98], and so $(\mathrm{V})$ is equivalent to the condition for extension given in [ABH02, Thm. 3.2(1), (4)].

Remark 5.8. Recall from Remark 2.7 that the following statement also holds for stacks. The Prym map $P^{\Sigma}: \overline{\mathcal{R}}_{g+1} \rightarrow \overline{\mathcal{A}}_{g}^{\Sigma}$ extends to a morphism in a neighborhood of an admissible cover $\pi: \widetilde{C} \rightarrow C$ if and only if there exists a cone $\sigma \in \Sigma_{\widetilde{C} / C}$ of the admissible decomposition containing the monodromy cone $\sigma(\widetilde{C} / C)$.

\section{Monodromy cones for Friedman-Smith covers}

We now investigate a class of admissible covers discovered by Friedman and Smith [FS86], who used these examples to show that the Prym map does not extend to the second Voronoi, perfect cone or central cone compactifications. Alexeev, Birkenhake, and Hulek [ABH02] and Vologodsky [Vol02] then showed that these examples characterize the indeterminacy locus of the Prym map to the second Voronoi compactification. In this section we give a detailed description of the monodromy cone for these examples with the aim of giving a geometric characterization of the indeterminacy locus of the Prym map to the perfect and central cone compactifications. In the subsequent sections we will actually need some more elaborate monodromy computations for further degenerations of these examples. The method for obtaining these is the same as the one discussed here, and thus all such further computations will be given in the appendices. In the main body of the paper we will reference those combinatorial results as needed.

\subsection{Friedman-Smith covers}

A Friedman-Smith cover with $2 n \geq 2$ nodes (see also Figure 6) is an admissible cover $\pi: \widetilde{C} \rightarrow C$ such that

- $\widetilde{C}=\widetilde{C}_{1} \cup \widetilde{C}_{2}$ with $\widetilde{C}_{1}$ and $\widetilde{C}_{2}$ irreducible and smooth, and

$$
\widetilde{C}_{1} \cap \widetilde{C}_{2}=\left\{\tilde{p}_{1}^{+}, \tilde{p}_{1}^{-}, \ldots, \tilde{p}_{n}^{+}, \tilde{p}_{n}^{-}\right\},
$$

- $\iota \widetilde{C}_{i}=\widetilde{C}_{i}$ for $i=1,2$,

- $\iota \tilde{p}_{i}^{+}=\tilde{p}_{i}^{-}$for $i=1, \ldots, n$.

Remark 6.1. An admissible cover $\pi: \widetilde{C} \rightarrow C$ is called a degeneration of a FriedmanSmith cover with $2 n$ nodes if it can be obtained from a Friedman-Smith cover by a further degeneration. More precisely, an admissible cover $\pi: \widetilde{C} \rightarrow C$ is such a degeneration if and only if $\tilde{\widetilde{C}}=\widetilde{C}_{1} \cup \widetilde{C}_{2}$ with $\widetilde{C}_{1}$ and $\widetilde{C}_{2}$ connected (possibly reducible), $\widetilde{C}_{1} \cap \widetilde{C}_{2}=$ $\left\{\tilde{p}_{1}^{+}, \tilde{p}_{1}^{-} \ldots, \tilde{p}_{n}^{+} \tilde{p}_{n}^{-}\right\}, \iota \widetilde{C}_{i}=\widetilde{C}_{i}$ for $i=1,2$, and $\iota \tilde{p}_{i}^{+}=\tilde{p}_{i}^{-}$for $i=1, \ldots, n$. 
For later use, we denote by $F S_{n} \subseteq \bar{R}_{g+1}$ the locus of Friedman-Smith covers, and by $\overline{F S}_{n}$ its closure, i.e. the locus of degenerations of Friedman-Smith covers. A (degeneration of a) Friedman-Smith graph is a dual graph together with an involution, which can be obtained as the dual graph of a (degeneration of a) Friedman-Smith cover with induced involution.

Remark 6.2. The following is slightly stronger than a direct translation of the remark above into the language of graphs. A graph $\widetilde{\Gamma}$ with admissible involution $\iota$ is a degeneration of a Friedman-Smith graph with at least $2 n \geq 2$ edges if and only if $\widetilde{\Gamma}$ admits disjoint connected subgraphs $\widetilde{\Gamma}_{1}, \widetilde{\Gamma}_{2}$ connected by exactly $2 m \geq 2 n$ edges $\tilde{e}_{1}^{+}, \tilde{e}_{1}^{-}, \ldots, \tilde{e}_{m}^{+}, \tilde{e}_{m}^{-}$, with $\iota\left(\widetilde{\Gamma}_{i}\right)=\widetilde{\Gamma}_{i}(i=1,2)$, and $\iota \tilde{e}_{i}^{+}=\tilde{e}_{i}^{-}(i=1, \ldots, m)$, and furthermore $\widetilde{\Gamma}_{1}$ and $\widetilde{\Gamma}_{2}$ are not connected by a $\iota$-invariant path [Vol02, Lem. 1.2].

One can see that $\overline{F S}_{1}=\bigcup \delta_{i, g-i}$ and for $n \geq 2, \overline{F S}_{n}$ is codimension $n$ (if non-empty), and contained in the $n$-fold self-intersection of $\delta_{0}^{\prime}$. In $\overline{\mathcal{R}}_{g+1}$ there are $\lfloor(g-n+2) / 2\rfloor$ irreducible components of $F S_{n}$, determined by the pairs of genera $\left(g\left(C_{1}\right), g\left(C_{2}\right)\right)$ given by

$$
(1, g-n+1),(2, g-n), \ldots(\lfloor(g-n+2) / 2\rfloor,\lfloor(g-n+3) / 2\rfloor) .
$$

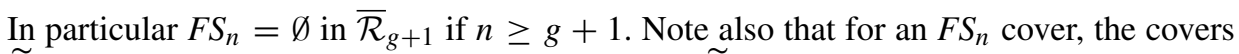
$\widetilde{C}_{i} \rightarrow C_{i}$ are étale, so that in particular the curves $\widetilde{C}_{i}$ have odd genus $2 g\left(C_{i}\right)-1$.

Remark 6.3. The index $n$ for $F S_{n}$ refers to the codimension of the locus in $\bar{R}_{g+1}$, or equivalently the number of edges in the dual graph of the base curve. We will use similar notational conventions for other loci occurring later in the paper.

\subsection{The monodromy cone}

We will now compute the monodromy cones associated to Friedman-Smith covers, which we will simply call Friedman-Smith cones, or FS cones. By an FS cone we will mean the monodromy cone associated to an $F S_{n}$ cover for some $n$. Let $\pi: \widetilde{C} \rightarrow C$ be a FriedmanSmith cover with $2 n \geq 2$ nodes. The dual graph $\widetilde{\Gamma}$ of $\widetilde{C}$ has vertices $V(\widetilde{\Gamma})=\left\{\tilde{v}_{1}, \tilde{v}_{2}\right\}$ and edges $E(\widetilde{\Gamma})=\left\{\tilde{e}_{1}^{+}, \tilde{e}_{1}^{-}, \ldots, \tilde{e}_{n}^{+}, \tilde{e}_{n}^{-}\right\}$. The involution $\iota$ acts by $\iota\left(\tilde{v}_{i}\right)=\tilde{v}_{i}(i=1,2)$ and $\iota\left(\tilde{e}_{i}^{+}\right)=\tilde{e}_{i}^{-}(i=1, \ldots, n)$. For simplicity, we will fix a compatible orientation on $\widetilde{\Gamma}$, as in Figure 6, i.e. for all $i$ set $t\left(\tilde{e}_{i}^{ \pm}\right)=\tilde{v}_{2}$ and $s\left(\tilde{e}_{i}^{ \pm}\right)=\tilde{v}_{1}$.
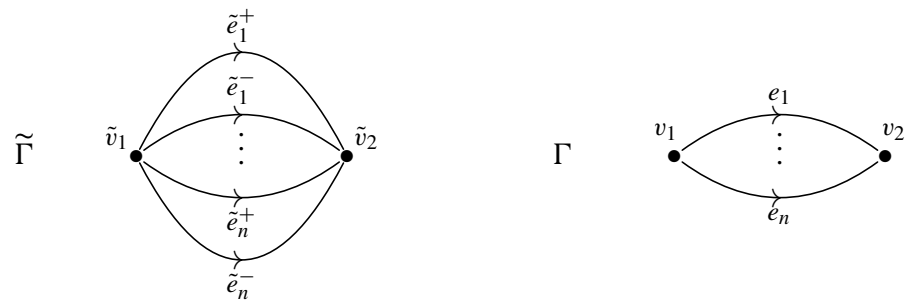

Fig. 6. Dual graph of a Friedman-Smith vover with $2 n \geq 2 \operatorname{nodes}\left(F S_{n}\right)$. 
One has

$$
H_{1}(\widetilde{\Gamma}, \mathbb{Z})=\mathbb{Z}\left\langle\tilde{e}_{1}^{+}-\tilde{e}_{1}^{-}, \ldots, \tilde{e}_{n}^{+}-\tilde{e}_{n}^{-}, \tilde{e}_{1}^{+}-\tilde{e}_{2}^{-}, \ldots, \tilde{e}_{n-1}^{+}-\tilde{e}_{n}^{-}\right\rangle .
$$

Indeed, we have $b_{1}(\widetilde{\Gamma})=\# E(\widetilde{\Gamma})-\# V(\widetilde{\Gamma})+b_{0}(\widetilde{\Gamma})=2 n-1$, since $\widetilde{\Gamma}$ is connected. The $2 n-1$ elements listed above are in fact a generating set for $H_{1}(\widetilde{\Gamma}, \mathbb{Z})$, as can be easily detected from the associated matrix. For instance, if one takes the elements in the order $\tilde{e}_{1}^{+}-\tilde{e}_{1}^{-}, \tilde{e}_{1}^{+}-\tilde{e}_{2}^{-}, \ldots, \tilde{e}_{n}^{+}-\tilde{e}_{n}^{-}, \tilde{e}_{n-1}^{+}-\tilde{e}_{n}^{-}$and constructs a matrix with rows expressing these elements with respect to the basis $\tilde{e}_{1}^{-}, \tilde{e}_{1}^{+}, \ldots, \tilde{e}_{n}^{-}, \tilde{e}_{n}^{+}$, one obtains a $(2 n-1) \times(2 n)$ matrix whose first $(2 n-1) \times(2 n-1)$ submatrix is upper triangular with all the diagonal entries equal to \pm 1 .

Recall that $H_{1}(\widetilde{\Gamma}, \mathbb{Z})^{[-]}=H_{1}(\widetilde{\Gamma}, \mathbb{Z}) / H_{1}(\widetilde{\Gamma}, \mathbb{Z})^{+}$and it is isomorphic to the image of the map

$$
\frac{1}{2}(\operatorname{Id}-\iota): H_{1}(\widetilde{\Gamma}, \mathbb{Z}) \rightarrow H_{1}(\widetilde{\Gamma}, \mathbb{R}) .
$$

From (6.1), one has

$H_{1}(\widetilde{\Gamma}, \mathbb{Z})^{[-]} \cong \mathbb{Z}\left\langle\tilde{e}_{1}^{+}-\tilde{e}_{1}^{-}, \frac{1}{2}\left(\tilde{e}_{1}^{+}-\tilde{e}_{1}^{-}\right)+\frac{1}{2}\left(\tilde{e}_{2}^{+}-\tilde{e}_{2}^{-}\right), \ldots, \frac{1}{2}\left(\tilde{e}_{n-1}^{+}-\tilde{e}_{n-1}^{-}\right)+\frac{1}{2}\left(\tilde{e}_{n}^{+}-\tilde{e}_{n}^{-}\right)\right\rangle$.

For brevity, set

$z_{1}=\tilde{e}_{1}^{+}-\tilde{e}_{1}^{-}, z_{2}=\frac{1}{2}\left(\tilde{e}_{1}^{+}-\tilde{e}_{1}^{-}\right)+\frac{1}{2}\left(\tilde{e}_{2}^{+}-\tilde{e}_{2}^{-}\right), \ldots, z_{n}=\frac{1}{2}\left(\tilde{e}_{n-1}^{+}-\tilde{e}_{n-1}^{-}\right)+\frac{1}{2}\left(\tilde{e}_{n}^{+}-\tilde{e}_{n}^{-}\right)$

so that $H_{1}(\widetilde{\Gamma}, \mathbb{Z})^{[-]} \cong \mathbb{Z}\left\langle z_{1}, \ldots, z_{n}\right\rangle$. Then

$$
H^{1}(\widetilde{\Gamma}, \mathbb{Z})^{-}=\left(H_{1}(\widetilde{\Gamma}, \mathbb{Z})^{[-]}\right)^{\vee} \cong \mathbb{Z}\left\langle z_{1}^{\vee}, \ldots, z_{n}^{\vee}\right\rangle .
$$

Now observe that

$H^{1}(\widetilde{\Gamma}, \mathbb{Z})=\mathbb{Z}\left\langle\left(\tilde{e}_{1}^{+}\right)^{\vee},\left(\tilde{e}_{1}^{-}\right)^{\vee}, \ldots,\left(\tilde{e}_{n}^{+}\right)^{\vee},\left(\tilde{e}_{n}^{-}\right)^{\vee}\right\rangle /\left\langle\left(\tilde{e}_{1}^{+}\right)^{\vee}+\left(\tilde{e}_{1}^{-}\right)^{\vee}+\cdots+\left(\tilde{e}_{n}^{+}\right)^{\vee}+\left(\tilde{e}_{n}^{-}\right)^{\vee}\right\rangle$.

It follows that for $i=1, \ldots, n$,

$$
\begin{array}{ll}
\iota\left(\tilde{e}_{i}^{+}\right)^{\vee}=\left(\tilde{e}_{i}^{-}\right)^{\vee}=-\left(\tilde{e}_{i}^{+}\right)^{\vee} & \text { if } n=1, \\
\iota\left(\tilde{e}_{i}^{+}\right)^{\vee}=\left(\tilde{e}_{i}^{-}\right)^{\vee} \neq-\left(\tilde{e}_{i}^{+}\right)^{\vee} \quad \text { if } n \geq 2 .
\end{array}
$$

Consequently, for $i=1, \ldots, n$ we may choose

$$
\ell_{e_{i}}:= \begin{cases}\left(\tilde{e}_{i}^{+}\right)^{\vee} & \text { if } n=1 \\ \left(\tilde{e}_{i}^{+}\right)^{\vee}-\left(\tilde{e}_{i}^{-}\right)^{\vee} & \text { if } n \geq 2 .\end{cases}
$$

For $n=1, \ell_{e_{1}}$ is clearly a basis for $H^{1}(\widetilde{\Gamma}, \mathbb{Z})^{-}$, and so condition $(\mathrm{V})$ of Theorem 5.6 holds in this case. Now consider the case $n \geq 2$. Evaluating the $\ell_{e_{i}}$ on the basis $z_{1}, \ldots, z_{n}$, we obtain

$$
\begin{aligned}
& \ell_{e_{1}}=2 z_{1}^{\vee}+z_{2}^{\vee}, \\
& \ell_{e_{2}}=\quad z_{2}^{\mathrm{V}}+z_{3}^{\vee} \text {, } \\
& \text { : } \quad \ddots \\
& \vdots \quad \ddots
\end{aligned}
$$

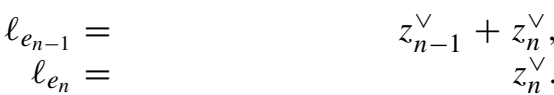


Thus, with respect to these bases, the matrix representation of the monodromy cone is

$$
\left(\begin{array}{cccccccc}
2 & 1 & & & & & & \\
& 1 & 1 & & & & & \\
& & 1 & 1 & & & & \\
& & & \ddots & \ddots & & & \\
& & & & & 1 & 1 & \\
& & & & & 1 & 1 \\
& & & & & & 1
\end{array}\right) .
$$

Since the determinant of this matrix is 2 , it follows that for $n \geq 2,\left\{\ell_{e_{1}}, \ldots, \ell_{e_{n}}\right\}$ is a basis for $H^{1}(\widetilde{\Gamma}, \mathbb{R})^{-}$, but is not a $\mathbb{Z}$-basis for $H^{1}(\widetilde{\Gamma}, \mathbb{Z})^{-}$. Consequently, condition (V) does not hold for $n \geq 2$.

\subsection{Properties of Friedman-Smith monodromy cones}

With the above description of the Friedman-Smith cones, it is now a combinatorial problem to describe the relationship of Friedman-Smith cones to the various cone decompositions. The details of the arguments are contained in the appendices. Here we compile the results for reference.

Theorem 6.4. A Friedman-Smith cone is:

(1) Basic for $n \geq 3$ and for $n=1$, and simplicial but not basic for $n=2$.

(2) Matroidal if and only if $n=1$. Every proper face of a Friedman-Smith cone is matroidal.

(3) Contained in a cone in the perfect cone decomposition if and only if $n \neq 2,3$. In fact, a Friedman-Smith cone is a cone in the perfect cone decomposition if and only if $n \neq 2,3,4$.

Proof. (1) See Lemma A.1. (2) See Lemma A.2. (3) See Proposition A.3.

Remark 6.5. In Appendix E, Dutour Sikirić shows that a Friedman-Smith cone is contained in a cone in the central cone decomposition if and only if $n \neq 2,3$.

Remark 6.6. It is an unpublished result of Alexeev that for $n \geq 2$, each cone in the barycentric subdivision of a Friedman-Smith cone is contained in a cone in the second Voronoi decomposition (see [Vol04, p. 3159]). It is easy to see that the decomposition of a Friedman-Smith cone into cones contained in second Voronoi cones must be a refinement of the barycentric subdivision. One can then argue as in Vologodsky [Vol04] to show that the barycentric subdivision suffices. We will use this in the case $n=2,3$ in our investigation of the resolution of the period map to the perfect cone compactification (see Remark 1.10). In the appendix (\$A.4) we give an explicit description of second Voronoi cones generated by rank 1 quadrics that decompose the Friedman-Smith cone for $n=2,3$, providing another proof in these special cases. We will use these explicit cones in studying other monodromy cones of degenerations of Friedman-Smith covers, and also in describing Delaunay decompositions. 


\section{The indeterminacy locus of the Prym map}

Here we further investigate the indeterminacy locus of the Prym map by reformulating the combinatorial characterization given in Theorem 5.6, in terms of geometry. For the second Voronoi compactification, Vologodsky [Vol02, Thm. 0.1] has shown that the combinatorial condition in Theorem 5.6(1) is equivalent to the cover being a degeneration of a Friedman-Smith cover with at least four nodes. In other words, the indeterminacy locus for the Prym map to the second Voronoi compactification is equal to $\bigcup_{n \geq 2} \overline{F S}_{n}$. Consequently, here we focus on the period map to the perfect cone compactification, for which it turns out that the indeterminacy locus is smaller. While at the moment we are unable to obtain a statement in full generality analogous to [Vol02, Thm. 0.1], we describe the situation completely up to codimension 6.

Theorem 7.1. The indeterminacy locus of the Prym map $P_{g}^{P}: \bar{R}_{g+1} \rightarrow \bar{A}_{g}^{P}$ satisfies

$$
\overline{F S}_{2} \cup \overline{F S}_{3} \subseteq \operatorname{Ind}\left(P_{g}^{P}\right) \subseteq \overline{F S}_{2} \cup \overline{F S}_{3} \cup \partial \overline{F S}_{4} \cup \cdots \cup \partial \overline{F S}_{g}
$$

where $\partial \overline{F S}_{n}=\overline{F S}_{n} \backslash F S_{n}$. Moreover,

$$
\operatorname{codim}_{\bar{R}_{g+1}} \operatorname{Ind}\left(P_{g}^{P}\right) \backslash\left(\overline{F S}_{2} \cup \overline{F S}_{3}\right) \geq 6 .
$$

Remark 7.2. Since $\bar{A}_{1}^{P}=A_{1}^{*}$, it is immediate (from the Borel extension theorem) that for $g=1$ the Prym map is a morphism $\bar{R}_{2} \rightarrow \bar{A}_{1}^{P}$; in this case both $F S_{2}$ and $F S_{3}$ are empty. For $g=2$ the locus $F S_{3}$ is empty, so we have $\operatorname{Ind}\left(P_{2}^{P}\right)=\overline{F S}_{2}$. Similarly, $\operatorname{Ind}\left(P_{3}^{P}\right)=\overline{F S}_{2} \cup \overline{F S}_{3}$. For $P_{4}^{P}: \bar{R}_{5} \rightarrow \bar{A}_{4}^{P}$ we have $\partial \overline{F S_{4}} \backslash\left(\overline{F S}_{2} \cup \overline{F S}_{3}\right) \neq \emptyset$. In this case, the theorem above says roughly that the "generic points" of this locus do not lie in the indeterminacy locus. We note that in the course of the proof we will obtain a slightly stronger result than the statement of the theorem, by showing that the Prym map extends to $\overline{F S}_{4}$ and $\overline{F S}_{5}$ up to codimension two.

Remark 7.3. We have the following relationships between the indeterminacy loci. For $g \leq 3, \operatorname{Ind}\left(P_{g}^{P}\right)=\operatorname{Ind}\left(P_{g}^{V}\right)=\operatorname{Ind}\left(P_{g}^{C}\right)$. For $g \geq 4, \operatorname{Ind}\left(P_{g}^{P}\right) \subsetneq \operatorname{Ind}\left(P_{g}^{V}\right)$. In Appendix E, Mathieu Dutour Sikirić shows that for $g \geq 4$, $\operatorname{Ind}\left(P_{g}^{C}\right) \nsupseteq \operatorname{Ind}\left(P_{g}^{V}\right)$, and for $g \geq 9, \operatorname{Ind}\left(P_{g}^{C}\right) \nsubseteq \operatorname{Ind}\left(P_{g}^{V}\right)$.

In the process of proving the results in this paper, we have considered a number of degenerations of Friedman-Smith covers. In these examples, the monodromy cone fails to be contained in a cone in the perfect cone decomposition (PCD) if and only if the example lies in $\overline{F S}_{2} \cup \overline{F S}_{3}$. We thus pose the following question.

Question 7.4. Is it true that the indeterminacy locus $\operatorname{Ind}\left(P_{g}^{P}\right)$ is equal to $\overline{F S}_{2} \cup \overline{F S}_{3}$ ?

Proof of Theorem 7.1. We start by showing

$$
\overline{F S}_{2} \cup \overline{F S}_{3} \subseteq \operatorname{Ind}\left(P_{g}^{P}\right) \subseteq \overline{F S}_{2} \cup \overline{F S}_{3} \cup \partial \overline{F S}_{4} \cup \cdots \cup \partial \overline{F S}_{g}
$$


Theorem 6.4(3) implies that the covers in the loci $F S_{2}, F S_{3}$ have monodromy cones not contained in cones in the PCD. This gives the left inclusion. For the right inclusion, the results [ABH02, Thm. 3.2 (1), (4)] and [Vol02, Thm. 0.1] imply that on $\bar{R}_{g+1} \backslash\left(\overline{F S}_{2} \cup \ldots\right.$ $\cup \overline{F S}_{g}$ ) the monodromy cones are matroidal, which by [MV12, Thm. A] are also perfect (see Remark 1.6). Moreover, in Theorem 6.4(3) we showed that for $4 \leq n \leq g$ a cover in $F S_{n}$ has a monodromy cone contained in a cone in the PCD, and thus the period map extends there as well.

We now prove

$$
\operatorname{codim}_{\bar{R}_{g+1}} \operatorname{Ind}\left(P_{g}^{P}\right) \backslash\left(\overline{F S}_{2} \cup \overline{F S}_{3}\right) \geq 6
$$

Since $\operatorname{codim} \overline{F S}_{n}=n$, it is enough to restrict attention to $\partial \overline{F S}_{4}$. In fact we will show the stronger statement that

$$
\operatorname{codim}_{\overline{F S}_{n}}\left(\operatorname{Ind}\left(P_{g}^{P}\right) \cap \overline{F S}_{n}\right) \backslash\left(\overline{F S}_{2} \cup \overline{F S}_{3}\right) \geq 2
$$

for $n=4,5$. To achieve this, we simply need to enumerate the codimension 1 degenerations in $\overline{F S}_{n}$ for $n=4,5$ and check that for each of them the monodromy cone is not contained in a cone in the PCD if and only if the degeneration also lies in $\overline{F S}_{2}$ or $\overline{F S}_{3}$. To be precise, we will consider all degenerations of an $\overline{F S}_{n}$ cover so that the dual graph of the base curve has exactly $n+1$ edges. The complement of this locus has codimension 2 in $\overline{F S}_{n}$. We observe that the dual graph of the base of a degeneration of an $\overline{F S}_{n}$ cover has exactly $n+1$ edges if and only if the dual graph of the covering curve is obtained by replacing a vertex in the dual graph of an $F S_{n}$ cover (see Figure 6) with one of the dual graphs in Figures 1-5. Thus we have five cases to consider.

First consider the case where we replace the vertex with a graph as in Figure 2 (corresponding to $\delta_{0}^{\prime \prime}$ ). This give rise to an $F S_{n}+W_{1}$ example (see Figure 11 with $m=1$ ). The monodromy computation is made in $\S \mathrm{C} .2$, and establishes Lemma C.4 stating that for $n \leq 7$, the monodromy cone is contained in a cone in the PCD if and only if the cover is not a degeneration of an $F S_{2}$ or $F S_{3}$ cover.

Next consider the case where we replace the vertex with a graph as in Figure 5 (corresponding to $\delta_{i, g+1-i}$ ). This gives rise to $F S_{n_{1}+n_{2}}+F S_{1}$ examples with $n_{1}+n_{2}=n$ (see Figure 14 with $m=1$ ). The monodromy computation is made in $\S$ C.3, and establishes Lemma C.7 stating that the monodromy cone is contained in a cone in the PCD if and only if the cover is not a degeneration of an $F S_{2}$ or $F S_{3}$ cover.

Next consider the case where we replace the vertex with a graph as in Figure 4 (corresponding to $\delta_{i}$ ). This gives rise to $F S_{n_{1}+n_{2}}+\delta_{i}$ examples with $n_{1}+n_{2}=n$ (see Figure 16). The monodromy computation is made in $\S$ C.4, culminating in Lemma C.11, which shows that for $n \leq 5$, the monodromy cone is contained in a cone in the PCD if and only if the cover is not a degeneration of an $F_{2}$ or $F S_{3}$ cover.

The cases where we replace the vertex with a graph as in Figure $3\left(\delta_{0}^{\mathrm{ram}}\right)$ or Figure 1 $\left(\delta_{0}^{\prime}\right)$ are similar. These give rise to $F S_{n}+B_{1}$ (resp. $F S_{n}+E E_{1}$ ) examples (see $\S$ C.5, resp. §C.6). Lemmas C.12 and C.13 show that the monodromy cone is contained in a cone in the PCD if and only if the cover is not a degeneration of an $F S_{2}$ or $F S_{3}$ cover. 


\section{Resolving the Prym map}

As discussed previously, in contrast to the case of the Torelli map for curves, the Prym map is not regular along (certain components of) the Friedman-Smith locus. For geometric applications (e.g. the study of moduli of cubic threefolds) it is important to have a regular map. Using the fact that the normal crossing compactifications and the toroidal compactifications have a toric structure at the boundary, it is always possible to refine the normal crossing compactification (by further toric blow-ups) to get a regular map. In this section we resolve the Prym map up to codimension 4. In the appendices we have worked out some further special cases in all genera for the perfect cone compactification; some special cases in all genera have also been considered by Alexeev and Vologodsky for the second Voronoi compactification (see §C.1 and [Vol04]).

Theorem 8.1. There is a closed locus $Z \subseteq \bigcup_{n=2}^{g} \partial \overline{F S}_{n} \subseteq \bar{R}_{g+1}$ with $\operatorname{codim}_{\bar{R}_{g+1}} Z \geq 4$ such that the restriction to $U=\bar{R}_{g+1} \backslash Z$ of the Prym period map $P_{g}^{P}: \bar{R}_{g+1} \rightarrow-\bar{A}_{g}^{P}$ can be resolved in the following way:

(1) The period map is regular on $U \backslash\left(\overline{F S}_{2} \cup \overline{F S}_{3}\right)$.

(2) If $x \in U \cap \overline{F S}_{2}$, then étale locally there are either one, two, or three components of $\overline{F S}_{2}$ meeting at $x$. If there are one or two components meeting, the period map is resolved by blowing up the union of the components. If there are three components meeting at $x$, the period map is resolved by the toric morphism determined by Figure (8.1).

(3) We have $U \cap \overline{F S}_{3}=F S_{3}$, and at a point $x \in F S_{3}$ the period map is resolved by blowing up the locus $\mathrm{FS}_{3}$.

In addition, for $g=2$ the period map $\bar{R}_{3} \rightarrow \bar{A}_{2}^{P}\left(=\bar{A}_{2}^{V}, \bar{A}_{2}^{C}\right)$ is resolved simply by blowing up $\overline{F S}_{2}$, which is irreducible (globally and étale locally).

Remark 8.2. One may take $Z$ in the theorem above so that for $n \geq 4, U \cap \overline{F S}_{n}=F S_{n}$. Then if in addition one blows up along $F S_{n}$ for $n \geq 4$, this resolves the period map on $U$ to the second Voronoi compactification.

Remark 8.3. In the appendix, we provide explicit resolutions of the period map to $\bar{A}_{g}^{P}$ for many more types of degenerations of Friedman-Smith covers. While these still do not cover enough special cases to resolve the period map $\bar{R}_{g+1} \rightarrow-\bar{A}_{g}^{P}$ for any $g \geq 3$, in principle, these computations could be carried out further to completely resolve the period map for low $g$.

Remark 8.4. In part (2) of the theorem, in the case where three components of $\overline{F S}_{2}$ meet, we point out that the birational modification is not the blow-up of the union of the three components, nor is it obtained by blowing up the intersection of the three components, followed by blowing up the strict transforms of the components (and neither of these birational modifications resolves the period map). 
Proof of Theorem 8.1. First let us define the locus $Z$ in the statement of the theorem. Let $Z_{2} \subseteq \partial \overline{F S}_{2}$ be the locus of degenerations whose dual graph is not obtained by replacing a vertex in the dual graph of an $F S_{2}$ cover (see Figure 6) with one of the dual graphs in Figures 1-5. Let

$$
Z=Z_{2} \cup \bigcup_{n=3}^{g} \partial \overline{F S}_{n} .
$$

Thus $\operatorname{codim} Z \geq 4$, and on $U$ the period map only fails to be regular along $U \cap \overline{F S}_{2}$ and $U \cap \overline{F S}_{3}=F S_{3}$. From Remark 6.6, at points of $F S_{2}$ and $F S_{3}$, the period map is resolved in a neighborhood by a blow-up of the $F S$ locus. The proof now proceeds in a similar fashion to the proof of Theorem 7.1. We enumerate the dual graphs obtained from covers in $U \cap \partial \overline{F S}_{2}$, and for each of them decompose the corresponding monodromy cone into cones in the PCD. Recall that this provides a resolution of the period map in the following way. Given an admissible double cover $\widetilde{C} \rightarrow C$, the miniversal space has snc boundary with components in bijection with edges of the dual graph $\Gamma$ of $C$. Consequently, for each edge $e$ of $\Gamma$, there is a corresponding quadratic form obtained via the log of monodromy. This induces a map from the standard simplex with vertices indexed by the edges of $\Gamma$, to the closure of the monodromy cone. Decomposing the monodromy cone into cones contained in the admissible cone decomposition, and then pulling back to the standard simplex, gives a decomposition of the standard simplex, which determines the minimal resolution of the period map in a neighborhood of the admissible cover $\widetilde{C} \rightarrow C$.

We now proceed to implement this, using the same enumeration as in the proof of Theorem 7.1. For the case of an $F S_{2}+W_{1}$ cover $\widetilde{C} \rightarrow C$, the dual graph $\Gamma$ has three edges $e_{1}, e_{2}, f$. The cone decomposition is given in $\S \mathrm{C} .2$ and has the form
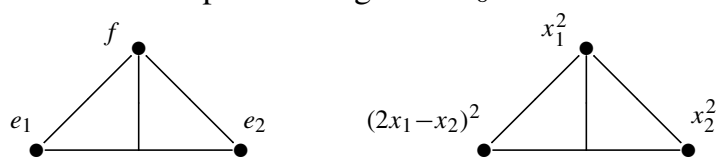

where $e_{1} \mapsto\left(2 x_{1}-x_{2}\right)^{2}, e_{2} \mapsto x_{2}^{2}$ and $f \mapsto x_{1}^{2}$. Étale locally, the divisors corresponding to $f, e_{1}$, and $e_{2}$ are all of type $\delta_{0}^{\prime}$. The intersection of the two copies of $\delta_{0}^{\prime}$ corresponding to $e_{1}$ and $e_{2}$ is exactly the locus of Friedman-Smith covers, which are of type $\overline{F S}_{2}$. The decomposition above indicates that this locus is blown up in the minimal resolution.

For the case of an $F S_{2+0}+F S_{1}$ cover, the cone decomposition is given in $\S \mathrm{C} .3$ (see Figure 15) and (in similar notation to the example above) has the form
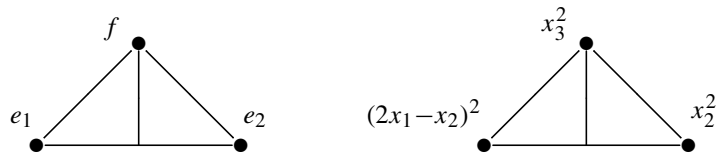

where $e_{1} \mapsto\left(2 x_{1}-x_{2}\right)^{2}, e_{2} \mapsto x_{2}^{2}$ and $f \mapsto x_{3}^{2}$. Étale locally, the divisors corresponding to $e_{1}$ and $e_{2}$ are of type $\delta_{0}^{\prime}$. The divisor corresponding to $f$ is of type $\delta_{1,1}$ (the $\overline{F S}_{1}$ locus). The intersection of the two copies of $\delta_{0}^{\prime}$ corresponding to $e_{1}$ and $e_{2}$ is exactly the locus of Friedman-Smith covers of type $\overline{F S}_{2}$. The decomposition above indicates that this locus is blown up in the minimal resolution. 
For the case of an $F S_{1+1}+F S_{1}$ cover it is shown in $§$ C.3 (see also §C.1 and [Vol04]) that the cone decomposition is (in similar notation)
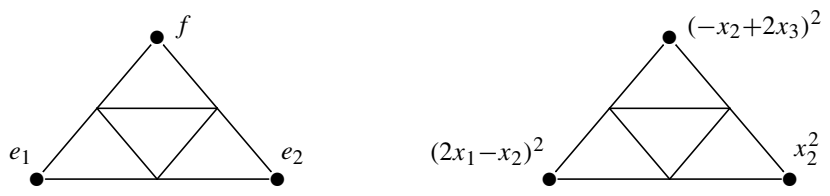

Étale locally, the divisors corresponding to $f, e_{1}$ and $e_{2}$ are of type $\delta_{0}^{\prime}$. In this case, each of the three pairwise intersections of these divisors is an $\overline{F S}_{2}$ locus. The associated birational modification that resolves the period map is an isomorphism away from this locus, and introduces three exceptional divisors. The corresponding birational modification of $\mathbb{A}_{\mathbb{C}}^{3}$ has as fiber over the origin equal to three copies of $\mathbb{P}_{\mathbb{C}}^{1}$ attached at a point.

For the $F S_{2+0}+\delta_{1}$ example, from the analysis in $\S \mathrm{C} .4$ we see that the cone decomposition is
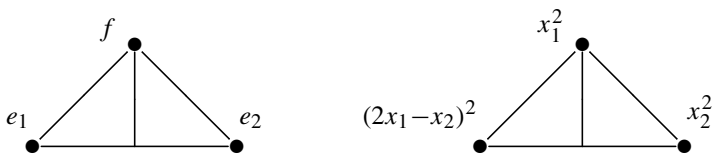

The divisors corresponding to $e_{1}, e_{2}$ and $f$ are of type $\delta_{0}^{\prime}$. The Friedman-Smith locus $\left(\overline{F S}_{2}\right)$ is given as the intersection of the two divisors corresponding to $e_{1}$ and $e_{2}$. The decomposition tells us that in the neighborhood of such a point, the minimal resolution is the blow-up of the $\overline{F S}_{2}$ locus.

For the $F S_{1+1}+\delta_{1}$ example, in $\S \mathrm{C} .4$ we see that the cone decomposition is given as
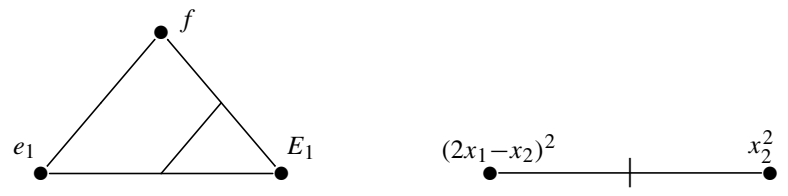

where $e_{1} \mapsto\left(2 x_{1}-x_{2}\right)^{2}, E_{1} \mapsto x_{2}^{2}$ and $f \mapsto\left(2 x_{1}-x_{2}\right)^{2}$. The divisors corresponding to $e_{1}, E_{1}$ and $f$ are of type $\delta_{0}^{\prime}$. In this case, the two components of the Friedman-Smith locus correspond to the intersection of the divisor corresponding to $e_{1}$ with the divisor corresponding to $E_{1}$, and also to the divisor corresponding to $f$ intersecting the divisor corresponding to $E_{1}$. These two loci are both of type $\overline{F S}_{2}$. The period map is resolved by blowing up the union of these loci.

For the $F S_{0+2}+\delta_{1}$ example, from the analysis in $\S C .4$ we see that the cone decomposition is
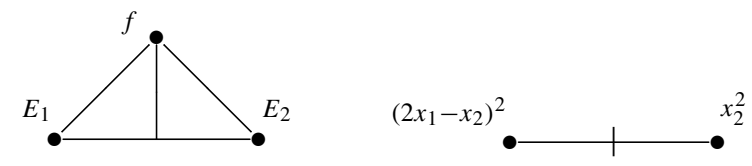

where $E_{1} \mapsto\left(2 x_{1}-x_{2}\right)^{2}, E_{2} \mapsto x_{2}^{2}$ and $f \mapsto 0$. The divisors corresponding to $E_{1}, E_{2}$ are of type $\delta_{0}^{\prime}$, while the divisor corresponding to $f$ is of type $\delta_{1}$. The Friedman-Smith locus $\left(\overline{F S}_{2}\right)$ is given as the intersection of the two divisors corresponding to $E_{1}$ and $E_{2}$. The 
decomposition tells us that in the neighborhood of such a point, the minimal resolution is the blow-up of the $\overline{F S}_{2}$ locus.

For the $F S_{2}+B_{1}$ example, from the analysis in $\S \mathrm{C} .5$ we see that the cone decomposition is
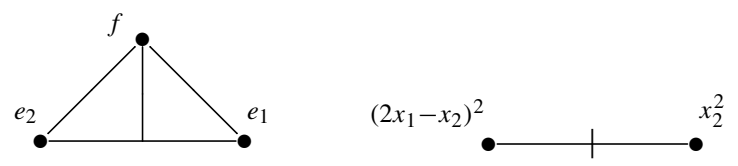

where $e_{1} \mapsto\left(2 x_{1}-x_{2}\right)^{2}, e_{2} \mapsto x_{2}^{2}$ and $f \mapsto 0$. The divisors corresponding to $e_{1}, e_{2}$ are of type $\delta_{0}^{\prime}$, while the divisor corresponding to $f$ is of type $\delta_{0}^{\text {ram }}$. The Friedman-Smith locus $\left(\overline{F S}_{2}\right)$ is given as the intersection of the two divisors corresponding to $e_{1}$ and $e_{2}$. The decomposition tells us that in the neighborhood of such a point, the minimal resolution is the blow-up of the $\overline{F S}_{2}$ locus.

For the $F S_{2}+E E_{1}$ example, from the analysis in $§ C .6$ we see that the cone decomposition is
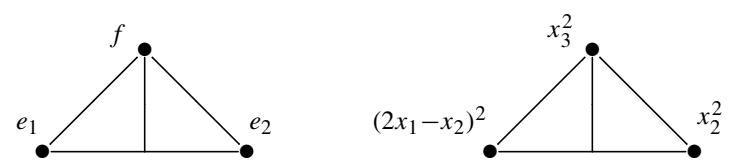

The divisors corresponding to $e_{1}, e_{2}$ and $f$ are of type $\delta_{0}^{\prime}$. The Friedman-Smith locus $\left(\overline{F S}_{2}\right)$ is given as the intersection of the two divisors corresponding to $e_{1}$ and $e_{2}$. The decomposition tells us that in the neighborhood of such a point, the minimal resolution is the blow-up of the $\overline{F S}_{2}$ locus.

The proof that the period map $\bar{R}_{3} \rightarrow-\bar{A}_{2}$ is resolved by blowing up $\overline{F S}_{2}$ is similar. There are more cases to consider, but in each case, the associated combinatorial data is a simplex that is star-subdivided along the edge corresponding to the two Friedman-Smith edges.

\section{Fibers of the resolution}

We now consider the question of describing the fibers of the resolution. We expect that with [Don92] and the techniques we describe here, it should be possible to give complete descriptions of the fibers over certain strata in low genus. We will pursue this elsewhere; here we limit ourselves to the following. Given a point $x \in \bar{A}_{g}$ in a given stratum, describe loci in the resolution of $\bar{R}_{g+1}$ that map to the same stratum. This can be rephrased in terms of 1-parameter families, which is what we actually consider. Moreover, since $\bar{A}_{g}^{V}, \bar{A}_{g}^{P}$ and $\bar{A}_{g}^{C}$ coincide outside $\bar{\beta}_{4}$ (torus rank 4 or more), for $\beta_{0} \cup \beta_{1} \cup \beta_{2} \cup \beta_{3}$ we can work with any one of them, e.g. we can adopt the language of the second Voronoi compactification as we shall do below.

\subsection{Degeneration data for Pryms}

Limits of 1-parameter families of ppavs are determined by degeneration data, which in turn determine the limit point in the toroidal compactification (see $\$ 2.4)$. Here we re- 
call from [ABH02] the degeneration data for Pryms. We begin with an admissible cover $\widetilde{C} \rightarrow C$, and a 1-parameter deformation associated to a map $\psi: S \rightarrow \operatorname{Def}_{\widetilde{C} / C}$, from the unit disk to the base of a miniversal deformation. Let $\Psi: S \rightarrow \bar{A}_{g}^{\Sigma}$ be the composition of $\psi$ with the rational map $\operatorname{Def}_{\widetilde{C} / C}-\rightarrow \bar{A}_{g}^{\Sigma}$.

First let us consider the degeneration data for the Jacobian of the covering curve $\widetilde{C}$. In this case, we saw in (3.1) that the generalized Jacobian $J \widetilde{C}$ corresponds to a morphism $\tilde{c}: H_{1}(\widetilde{\Gamma}, \mathbb{Z}) \rightarrow \widehat{J \widetilde{N}}$, where $\widetilde{N}$ is the normalization of $\widetilde{C}$. We recall the morphism $\tilde{c}$. A node on $\widetilde{C}$ corresponds to an edge $e$ in $C_{1}(\widetilde{\Gamma}, \mathbb{Z})=\bigoplus \mathbb{Z} \tilde{e}_{j}$ going from a vertex $\tilde{v}^{+}$to a vertex $\tilde{v}^{-}$. Let $\tilde{Q}_{+}(e)$ be the point corresponding to $\tilde{v}^{+}$in $\widetilde{N}$ and similarly for $\tilde{v}^{-}$(if $\tilde{v}^{+}=\tilde{v}^{-}$then it does not matter which of the two points above the double point is $\tilde{Q}_{+}(e)$ and which one is $\tilde{Q}_{-}(e)$ ). The map $\tilde{c}$ is defined by restricting the map

$$
\tilde{c}: C_{1}(\tilde{\Gamma}, \mathbb{Z}) \rightarrow J \tilde{N}_{0}, \quad e \mapsto \mathcal{O}\left(Q_{+}(e)\right) \otimes \mathcal{O}\left(Q_{-}(e)\right)^{-1},
$$

to the sublattice $H_{1}(\widetilde{\Gamma}, \mathbb{Z})$. The isomorphism $\phi: H^{1}(\widetilde{\Gamma}, \mathbb{Z}) \rightarrow H_{1}(\widetilde{\Gamma}, \mathbb{Z})$ is the canonical isomorphism, and $\hat{\tilde{c}}: H^{1}(\widetilde{\Gamma}, \mathbb{Z}) \rightarrow J \tilde{N}$ is defined as $\lambda^{-1} \circ c \circ \phi$, where $\lambda$ is the canonical principal polarization. The bihomomorphism $\tilde{\tau}$ is related to the Deligne symbol, and the quadratic form $\widetilde{B}$ is given by the valuation of $\tilde{\tau}$ or alternatively the log of monodromy computed in Proposition 4.1.

Now let us describe the degeneration data for the Prym. As discussed in (3.3), the generalized (open) Prym corresponds to a morphism $c^{-}: H_{1}(\widetilde{\Gamma}, \mathbb{Z})^{[-]} \rightarrow \widehat{A}$, where $A$ is a finite cover of the abelian variety

$$
P_{N}:=\operatorname{ker}(\mathrm{Nm}: J \tilde{N} \rightarrow J N)_{0}
$$

(see [ABH02, Prop. 1.5]). The map $c^{-}$is given by the commutative diagram

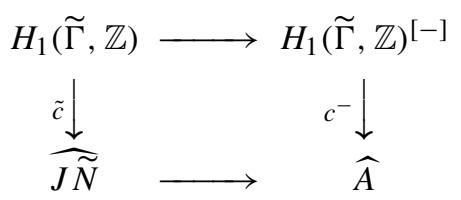

The bihomomorphism $\tau^{-}$is given by the "restriction" of the bihomomorphism $\tilde{\tau}$ [ABH02, $\S 3.2$, and $\$ 2.2$, p. 93]. The bilinear form $B^{-}$is again given by the valuation of $\tau^{-}$or equivalently the log of monodromy computed in Proposition 4.3.

In summary, $\Psi(0) \in \beta_{i}$ if and only if $\operatorname{rank} H_{1}(\widetilde{\Gamma}, \mathbb{Z})=i$, and $\Psi(0) \in \beta(\sigma)$ if and only if $B^{-} \in \sigma$ where $\sigma$ is the minimal cone with this property. The remaining modulus for $\Psi(0)$ is determined by the remaining degeneration data.

Note that by definition, $B^{-} \in \sigma(\widetilde{C} / C)$, the monodromy cone. If $\sigma(\widetilde{C} / C)$ is contained in a cone in $\Sigma$, then the Prym map extends in a neighborhood of $\widetilde{C} \rightarrow C$, and so $\Psi(0)$ depends only on $\widetilde{C} \rightarrow C$, and not on the 1-parameter family. Otherwise, a decomposition of $\sigma(\widetilde{C} / C)$ into cones in $\Sigma$ shows the dependence of even the combinatorial data on the 1-parameter family. More precisely, in the notation of Remark 4.4, $B^{-}=\sum_{e \in \Gamma}$ ord $\psi^{*}\left(z_{e}\right)\left(\tilde{e}^{\vee}-i \tilde{e}^{\vee}\right)^{2}$, and the order of vanishing determines the subcone of $\sigma(\widetilde{C} / C)$ in which the quadratic form $B^{-}$lies. We shall now illustrate the above discussion with several examples. 
As a final note, the combinatorics of rank 1 quadratic forms on a lattice are best considered by using squares of primitive rank 1 linear forms. This is the convention we use in the appendices. To match those descriptions to the ones in this section, it works best to describe the quadratic form $B^{-}$as

$$
B^{-}=\sum_{e \in \Gamma} \alpha_{e} \ell_{e}^{2}
$$

where $\ell_{e}$ is defined in (5.1) and

$$
\alpha_{e}:= \begin{cases}\operatorname{ord} \psi^{*}\left(z_{e}\right) & \text { if } \iota \tilde{e}^{\vee} \neq-\tilde{e}^{\vee} . \\ 4 \operatorname{ord} \psi^{*}\left(z_{e}\right) & \text { if } \iota \tilde{e}^{\vee}=-\tilde{e}^{\vee},\end{cases}
$$

\subsection{The Friedman-Smith loci $F S_{n}$}

In this section we consider the image of the strict transforms of the Friedman-Smith loci. That is, given a 1-parameter family of admissible covers, degenerating to a FriedmanSmith cover in $F S_{n}$, we want to describe the associated point in $\bar{A}_{g}^{V}$.

As we have already seen, the Friedman-Smith loci $F S_{n}$ consist of several components. These can be enumerated as follows: the curve $C$ is reducible, more precisely $C=C_{1} \cup C_{2}$ where $C_{1}$ and $C_{2}$ intersect in $n$ points. The curves $C_{i}$ are smooth and irreducible. If $g_{i}=$ $g\left(C_{i}\right)$, then $g_{1}+g_{2}+n-1=g+1$. The components of $F S_{n}$ then correspond to the different possibilities for $g_{1} \geq g_{2}>0$. From $\S 3$ and $\S 6$ one can see that $\operatorname{rank} H_{1}(\widetilde{\Gamma}, \mathbb{Z})^{[-]}=n$ and $A=P_{N}=P_{N_{1}} \times P_{N_{2}}$ (see also [ABH02]). This determines the image in $A_{g-1}^{*}$. For the remaining extension data, we will focus on the quadratic form $B^{-}$, starting with a general point on an irreducible component of $F S_{2}$.

9.2.1. The $\mathrm{FS}_{2}$ locus. Here we are in the torus rank 2 case, i.e. $i=2$. Thus we are no longer in Mumford's partial compactification, but we are still in the range where all known toroidal compactifications coincide, in particular also the second Voronoi compactification and the perfect cone compactification. In the notation of $\S$ A.4, where the decomposition of the monodromy cone is established, the form $B^{-}$is given by $\alpha_{1}\left(\tilde{e}_{1}^{\vee}-i \tilde{e}_{1}^{\vee}\right)^{2}+$ $\alpha_{2}\left(\tilde{e}_{2}^{\vee}-\imath \tilde{e}_{2}^{\vee}\right)^{2}$ with $\alpha_{i}=$ ord $\psi^{*}\left(z_{e_{i}}\right)$, and the monodromy cone decomposes as $\alpha_{1}<\alpha_{2}$, $\alpha_{1}=\alpha_{2}, \alpha_{1}>\alpha_{2}$. In the general case, $\alpha_{1}=\alpha_{2}$. As explained in Remark A.7, the associated Delaunay decomposition of $\mathbb{R}^{2}$ is that of squares and the corresponding cone is equivalent to the standard cone $\sigma_{1+1}=\left\langle x_{1}^{2}, x_{2}^{2}\right\rangle$. The degenerate Prym is in this case a $\mathbb{P}^{1} \times \mathbb{P}^{1}$-bundle over $A$ with "opposite" coordinate lines $\{0\} \times \mathbb{P}^{1}$ and $\{\infty\} \times \mathbb{P}^{1}$ as well as $\mathbb{P}^{1} \times\{0\}$ and $\mathbb{P}^{1} \times\{\infty\}$ identified with a shift over $A$. This shift is determined by $c^{-}$. There is a further parameter $b \in \mathbb{C}^{*}$ which describes the gluing of the lines which are identified. This parameter is given by the bihomomorphism $\tau$ and varies with the chosen 1-parameter family (even if $B^{-}$does not change). In fact, this is the parameter in the fibers of the $\mathbb{P}^{1}$-bundle given by blowing up the general point of an irreducible component of $\mathrm{FS}_{2}$.

We can also choose 1-parameter families with $\alpha_{1}>\alpha_{2}$ (the case $\alpha_{1}<\alpha_{2}$ can be obtained by symmetry). Now, as explained in Remark A.7, the Delaunay decomposition 
changes: every square breaks up into two triangles and the corresponding cone is equivalent to $\sigma_{K_{3}}=\left\langle x_{1}^{2}, x_{2}^{2},\left(x_{1}+x_{2}\right)^{2}\right\rangle$. The degenerate Prym is the union of two $\mathbb{P}^{2}$-bundles over $A$ with their coordinate lines identified appropriately again with a shift over $A$ which is determined by $c^{-}$. In this case the torus bundle $\mathcal{T}(\sigma)$ has rank 0 and the bihomomorphism $\tau$ is trivial. We shall see below that points in $\beta\left(\sigma_{1+1}\right)$ can also arise from other degenerations.

9.2.2. The $\mathrm{FS}_{3}$ locus. We shall now move on to general points on $F S_{3}$. As before the crucial point is the form $B^{-}$. In the notation of $\S A .4$, where the decomposition of the monodromy cone is established, the form $B^{-}$is given by $\sum_{i=1}^{3} \alpha_{i}\left(\tilde{e}_{i}^{\vee}-\imath \tilde{e}_{i}^{\vee}\right)^{2}$ with $\alpha_{i}=$ ord $\psi^{*}\left(z_{e_{i}}\right)$, and the monodromy cone decomposes as $\alpha_{1}=\alpha_{2}=\alpha_{3}, \alpha_{1}<\alpha_{2}=\alpha_{3}$, $\alpha_{1}<\alpha_{2}<\alpha_{3}$, together with all permutations. Here we discuss the general case, $\alpha_{1}=$ $\alpha_{2}=\alpha_{3}$. In Remark A.8 is is shown that the associated cone is equivalent to $\sigma_{C_{4}}=$ $\left\langle x_{1}^{2}, x_{2}^{2}, x_{3}^{2},\left(x_{1}+x_{2}+x_{3}\right)^{2}\right\rangle$. The associated Delaunay decomposition of $\mathbb{R}^{3}$ consists of one octahedron and two tetrahedra. The homomorphism $c^{-}$again defines shift parameters and $\tau$ defines gluing parameters (see [GH11, Section 7.2]). The latter depends on the 1-parameter family. The 1-parameter families with different orders of vanishing will result in $B^{-}$lying in one of the other cones in the decomposition of the monodromy cone (see Remark A.8).

Remark 9.1. The interesting point to note here is that this is a codimension 1 stratum in $\beta_{2}^{\Sigma}$, and hence the exceptional divisors over the general points of the irreducible components of $\mathrm{FS}_{3}$ do not map dominantly to $\beta_{2}^{\Sigma}$, even for small $g$. Thus the question remains to find an example which maps to the (unique) maximal stratum in $\beta_{2}^{\Sigma}$, namely the stratum $\beta\left(\sigma_{1+1+1}\right)$ where $\sigma_{1+1+1}=\left\langle x_{1}^{2}, x_{2}^{2}, x_{3}^{2}\right\rangle$. Indeed, this is not difficult to find. We can take an elementary étale covering with six nodes (discussed below).

Remark 9.2. More generally, for an $F S_{n}$ locus with $n \geq 2$, if both $g_{1}, g_{2}>1$, then the abelian variety $A$ is reducible. Hence the strict transforms of the corresponding FriedmanSmith loci cannot map dominantly onto $\beta_{i}$, even when mapping to the relevant stratum $A_{g-n}$ of $A_{g}^{*}$. For the remaining stratum $g_{2}=1$, it is possible that the map from this component of $F S_{2}$ to $\beta_{2}$ is dominant for $g \leq 5$. This is clear for $g=2$, but in general needs some discussion of the continuous parameters. The main issue is whether the projection under $q$ (see $\$ 2.4)$ maps surjectively to $\mathcal{X}^{\times 2}$.

\subsection{Elementary étale examples}

In this section we show that the elementary étale examples (see §B.2) map to the (unique) maximal stratum in $\beta_{2}^{\Sigma}$, namely the stratum $\beta\left(\sigma_{1+1+1}\right)$ where $\sigma_{1+1+1}=\left\langle x_{1}^{2}, x_{2}^{2}, x_{3}^{2}\right\rangle$. Indeed, it is shown in $\S \mathrm{B} .2$ that the monodromy cone of an elementary étale example with $2 n$ nodes is of type $\sigma_{1+\cdots+1}$. The associated degenerate abelian varieties are $\left(\mathbb{P}^{1}\right)^{n}$ bundles over abelian varieties with "opposite sides" glued with a shift. The shift is given by $c^{-}$, the gluing by $\tau$. In particular points in $\beta\left(\sigma_{1+1}\right)$ can arise not only from $F S_{2}$ covers but also from elementary étale covers with four nodes. 


\subsection{The Wirtinger locus $W_{n}$}

We conclude this discussion with the Wirtinger examples $W_{n}$ (see $\S$ B.3). In this case $\widetilde{C}$ has two components which are exchanged by the involution and $2 n$ nodes which are pairwise interchanged. In Remark B.2 it is established that the $W_{n}$ monodromy cone is $\mathbb{R}_{\geq 0}\left\langle x_{1}^{2},\left(x_{1}-x_{2}\right)^{2}, \ldots,\left(x_{n-2}-x_{n-1}\right)^{2}, x_{n-1}^{2}\right\rangle$. For $n=3$, the Delaunay decomposition of $\mathbb{R}^{2}$ is the tiling by 2-simplices obtained by slicing the standard square into two triangles. The associated degenerate abelian variety is a union of two copies of $\mathbb{P}^{2}$-bundles, corresponding to the slicing of the square into two triangles. As always the gluing is determined by $c^{-}$, the bihomomorphism $\tau$ is trivial here. We also see that points in $\beta\left(\sigma_{K_{3}}\right)$ can arise both from the blow-up of $F S_{2}$ and from Wirtinger examples $W_{3}$. For $n=4$ the toric part of the semiabelic variety corresponds to the dicing of a cube into an octahedron and two tetrahedra, and thus consists of a complete intersection $F(2,2)$ of two quadrics in $\mathbb{P}^{5}$ and two copies of $\mathbb{P}^{3}$. We take an opportunity to correct here an error in [ABH02, Example 5.2.2] where it was claimed that the toric parts are all projective spaces. We note that the general $F S_{3}$ degenerations are mapped to the same stratum.

\section{Appendix A. Combinatorics of Friedman-Smith cones}

In this appendix we establish various combinatorial properties of the monodromy cones of Friedman-Smith covers. Our starting point will be the computation in $\$ 6.2$, which culminated in (6.3) giving a description of the monodromy cone in matrix form. We first note that changing basis, one may take the Friedman-Smith cone for a cover of type $F S_{n}$, $n \geq 2$, to be generated by the quadratic forms that are given by the squares of the linear forms determined by the rows of the following matrix:

$$
F S_{n} \quad\left(\begin{array}{cccccccc}
2 & -1 & -1 & & & & -1 \\
0 & 1 & 0 & & & & 0 \\
0 & 0 & 1 & 0 & & & \\
& & & \ddots & \ddots & & \\
& & & 0 & 1 & 0 & 0 \\
& & & & 0 & 1 & 0 \\
& & & & & 0 & 1
\end{array}\right)
$$

This matrix can be obtained from (6.3) by integral column operations, and by replacing rows with their negatives.

\section{A.1. Friedman-Smith cones, simplicial cones, and basic cones}

Lemma A.1. The Friedman-Smith cones are basic for $n \geq 3$ and for $n=1$, and simplicial but not basic for $n=2$.

Proof. For $n=1$ the assertion is clear. For $n \geq 2$ we use the description in (A.1). For $n=2$ the forms $\left(2 x_{1}-x_{2}\right)^{2}$ and $x_{2}^{2}$ are independent, hence the cone is simplicial. On the other hand, these forms are not part of a basis since they do not span a primitive sublattice: the difference $\left(2 x_{1}-x_{2}\right)^{2}-x_{2}^{2}$ is not primitive. For $n \geq 3$ the situation is different. Adding the forms $x_{1}^{2},\left(x_{1}-x_{i}\right)^{2}, i=2, \ldots, n$, and $\left(x_{i}-x_{j}\right)^{2}, 2 \leq i<j \leq n,(i, j) \neq(2,3)$, one obtains a basis of $\operatorname{Sym}^{2} \mathbb{Z}^{n}$, and thus the cone is basic. 


\section{A.2. Friedman-Smith cones and second Voronoi cones}

Lemma A.2. A Friedman-Smith cone is matroidal if and only if $n=1$. Every proper face of a Friedman-Smith cone is matroidal.

Proof. The first statement follows from $\$ 6.2$ and (A.1) (the determinant is 2 if $n>1$ ). The second follows since any $n-1$ rows of the matrix (A.1) can be extended to a $\mathbb{Z}$-basis of $\mathbb{Z}^{n}$ (this also shows that every face of a Friedman-Smith monodromy cone is contained in a cone of type $A-$ see (1.5)).

\section{A.3. Friedman-Smith cones and perfect cones}

Proposition A.3. A Friedman-Smith cone is contained in a cone in the perfect cone decomposition if and only if $n \neq 2,3$. In fact, it is a cone in the perfect cone decomposition if and only if $n \neq 2,3,4$.

Remark A.4. In Appendix E, Dutour Sikirić shows that a Friedman-Smith cone is contained in a cone in the central cone decomposition if and only if $n \neq 2,3$. His proof also shows that a Friedman-Smith cone is contained in a cone in the perfect cone decomposition if and only if $n \neq 2,3$. The proof we give below establishes, for the PCD, the stronger statement that a Friedman-Smith cone is a cone in the PCD if and only if $n \neq 2,3,4$.

Proof of Proposition A.3. Let $\Lambda=\mathbb{Z}^{n}$ and fix the standard basis $e_{1}, \ldots, e_{n}$. Let $e_{1}^{\vee}, \ldots, e_{n}^{\vee}$ be the standard dual basis for $\Lambda^{\vee}$. First let us show that the FriedmanSmith cones, the cones generated by the quadratic forms $\left(2 e_{1}^{\vee}-e_{2}^{\vee}-\ldots-e_{n}^{\vee}\right)^{2}$, $\left(e_{2}^{\vee}\right)^{2}, \ldots,\left(e_{n}^{\vee}\right)^{2}$, are contained in a cone in the perfect cone decomposition if and only if $n \neq 2,3$.

For $n=1$, the cone is clearly matroidal, so it is a cone in the perfect cone decomposition (e.g. Remark 1.6). For lattices of rank 2, 3, every cone in the perfect cone decomposition is also a matroidal cone (see Remark 1.9). Consequently, the fact that the Friedman-Smith cone is generated by rank 1 quadrics and is not matroidal (Lemma A.2) implies it is not contained in a matroidal cone (Lemma 1.2), and hence not contained in a cone in the perfect cone decomposition.

For $n \geq 4$, consider the metric on $\mathbb{R}^{n}$ induced by the matrix

$$
Q=\left(\begin{array}{ccccc}
n / 4 & 1 / 2 & \ldots & 1 / 2 & 1 / 2 \\
1 / 2 & 1 & 0 & \ldots & 0 \\
\vdots & & \ddots & & \\
\vdots & & & \ddots & \\
1 / 2 & 0 & \ldots & 0 & 1
\end{array}\right)
$$

The matrix has determinant $1 / 4$, and so is clearly positive definite (consider leading principal minors starting from say the bottom right corner). The metric takes value 1 on $2 e_{1}^{\vee}-e_{2}^{\vee}-\cdots-e_{n}^{\vee}, e_{2}^{\vee}, \ldots, e_{n}^{\vee}$. Thus the Friedman-Smith cone is contained in a cone in the perfect cone decomposition for every $n \geq 4$, provided we can show that $Q$ takes values at least 1 on all non-zero elements of $\Lambda^{\vee}$. This is immediate by inspection for 
$n \equiv 0(\bmod 4)$; we will prove this in general by describing the cones determined by $Q$ in more detail.

To this end, consider another copy of $\mathbb{Z}^{n}$ with basis $f_{1}, \ldots, f_{n}$. We will also use the standard basis $f_{1}^{\vee}, \ldots, f_{n}^{\vee}$ for $\left(\mathbb{Z}^{n}\right)^{\vee}$. Inside of $\left(\mathbb{R}^{n}\right)^{\vee}$ consider the lattice $\mathbb{L}$ generated by $\ell=\frac{1}{2} f_{1}^{\vee}+\cdots+\frac{1}{2} f_{n}^{\vee}$, and $f_{2}^{\vee}, \ldots, f_{n}^{\vee}$. We have $\left(\mathbb{Z}^{n}\right)^{\vee} \subseteq \mathbb{L}$, since

$$
f_{1}^{\vee}=2 \ell-f_{2}^{\vee}-\cdots-f_{n}^{\vee} .
$$

Let $Q_{0}$ be the standard quadratic form on $\left(\mathbb{R}^{n}\right)^{\vee}$ :

$$
Q_{0}=f_{1}^{2}+\cdots+f_{n}^{2} .
$$

For simplicity, let us work momentarily in coordinates. It is clear that any vector

$$
\left(a_{1}, \ldots, a_{n}\right) \in\left(\mathbb{R}^{n}\right)^{\vee}
$$

will have length greater than 1 if any of the coefficients $a_{i}$ has magnitude greater than 1 , or any one coefficient has magnitude 1 and any other coefficient is non-zero. We can then easily enumerate the non-zero elements of $\mathbb{L}$ that do not satisfy these conditions:

$$
( \pm 1,0, \ldots, 0),(0, \pm 1,0, \ldots, 0), \ldots,(0, \ldots, 0, \pm 1),( \pm 1 / 2, \ldots, \pm 1 / 2) .
$$

Since

$$
Q_{0}( \pm 1 / 2, \ldots, \pm 1 / 2)=n / 4,
$$

we see that the non-zero elements of $\mathbb{L}$ of minimal length are:

- $( \pm 1 / 2, \ldots, \pm 1 / 2), n<4$,

- $( \pm 1,0, \ldots, 0),(0, \pm 1,0, \ldots, 0), \ldots,(0, \ldots, 0, \pm 1),( \pm 1 / 2, \ldots, \pm 1 / 2), n=4$,

- $( \pm 1,0, \ldots, 0),(0, \pm 1,0, \ldots, 0), \ldots,(0, \ldots, 0, \pm 1), n>4$.

Now consider the isomorphism $\Lambda^{\vee} \rightarrow \mathbb{L}$ given by $e_{1}^{\vee} \mapsto \ell, e_{2}^{\vee} \mapsto f_{2}^{\vee}, \ldots, e_{n}^{\vee} \mapsto f_{n}^{\vee}$. The pull-back of $Q_{0}$ under this isomorphism is $Q$, and the pull-back of the basis elements $f_{1}^{\vee}, \ldots, f_{n}^{\vee}$ are the elements $2 e_{1}^{\vee}-e_{2}^{\vee}-\cdots-e_{n}^{\vee}, e_{2}^{\vee}, \ldots, e_{n}^{\vee}$. Thus for $n>4$, the Friedman-Smith cone is exactly the cone in the perfect cone decomposition determined by $Q$. For $n=4$, the cone determined by $Q$ has 12 extremal rays, and is in fact a type $D$ cone (see (1.5)). One can show by enumerating the faces of a type $D$ cone in dimension 4 that the Friedman-Smith cone is not a face (and thus is not a cone in the perfect cone decomposition).

\section{A.4. Cone decompositions for $\mathrm{FS}_{2}$ and $\mathrm{FS}_{3}$}

Lemma A.5. For $n=2,3$, each cone in the barycentric subdivision of the FriedmanSmith cone is contained in a matroidal cone (and thus also in a cone in the perfect cone decomposition and the central cone decomposition). 
Remark A.6. This lemma follows from the fact (see Remark 6.6) that each cone in the barycentric subdivision of a Friedman-Smith cone is contained in a cone in the second Voronoi decomposition (since for $n=2,3$ second Voronoi cones are matroidal). However, we will want to know the exact cones containing the cones in the barycentric subdivision for later computations.

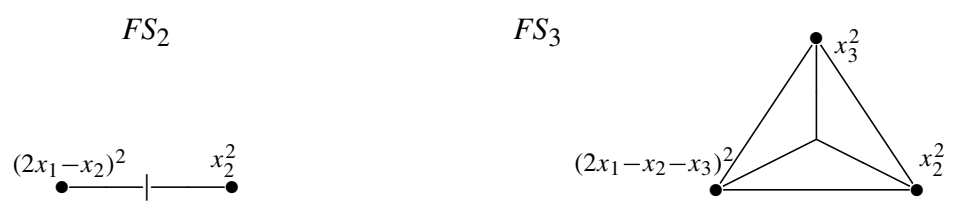

Fig. 7. Decomposition of the monodromy cone for $F S_{2}$ and $F S_{3}$ covers.

Proof of Lemma A.5. First consider the case $n=2$. The monodromy cone is

$$
\mathbb{R}_{\geq 0}\left\langle\left(2 x_{1}-x_{2}\right)^{2}, x_{2}^{2}\right\rangle \text {. }
$$

Consider the cones

$$
C_{1}=\mathbb{R}_{\geq 0}\left\langle x_{1}^{2}, x_{2}^{2},\left(x_{1}-x_{2}\right)^{2}\right\rangle \text { and } C_{2}=\mathbb{R}_{\geq 0}\left\langle\left(2 x_{1}-x_{2}\right)^{2}, x_{1}^{2},\left(x_{1}-x_{2}\right)^{2}\right\rangle .
$$

One can easily check (by say computing all the minors of the associated matrices) that these cones are matroidal. In fact, they are of type $A$ (see (1.5)). Now clearly $x_{2}^{2}$ is contained in $C_{1}$ and $\left(2 x_{1}-x_{2}\right)^{2}$ is contained in $C_{2}$. It only remains to check that the ray dividing the monodromy cone, generated by

$$
\left(2 x_{1}-x_{2}\right)^{2}+x_{2}^{2}
$$

is in both cones. We have

$$
\frac{1}{2}\left(\left(2 x_{1}-x_{2}\right)^{2}+x_{2}^{2}\right)=\frac{1}{2}\left(\left(4 x_{1}^{2}-4 x_{1} x_{2}+x_{2}^{2}\right)+x_{2}^{2}\right)=x_{1}^{2}+\left(x_{1}-x_{2}\right)^{2} .
$$

Thus the central ray of the monodromy cone is also the central ray of the common face of $C_{1}$ and $C_{2}$.

Now consider the case $n=3$. The monodromy cone is

$$
\mathbb{R}_{\geq 0}\left\langle\left(2 x_{1}-x_{2}-x_{3}\right)^{2}, x_{2}^{2}, x_{3}^{2}\right\rangle
$$

Motivated by the previous example, let us first see if we can find a cone that contains $x_{2}^{2}, x_{3}^{2}$ and also the middle ray of the cone, generated by

$$
\left(2 x_{1}-x_{2}-x_{3}\right)^{2}+x_{2}^{2}+x_{3}^{2} .
$$

Let $C_{1}$ be the cone

$$
C_{1}=\mathbb{R}_{\geq 0}\left\langle x_{1}^{2}, x_{2}^{2}, x_{3}^{2},\left(x_{1}-x_{2}\right)^{2},\left(x_{1}-x_{3}\right)^{2},\left(x_{1}-x_{2}-x_{3}\right)^{2}\right\rangle .
$$

It is somewhat tedious, but one can easily check (by say computing all the minors of the associated matrix) that this cone is matroidal. In fact, this is equivalent to the principal or 
$A_{n}$-cone in genus 3 , and every cone in the perfect cone decomposition is equivalent to a face of this cone. Computing, we have

$$
\left(2 x_{1}-x_{2}-x_{3}\right)^{2}+x_{2}^{2}+x_{3}^{2}=4 x_{1}^{2}+2 x_{2}^{2}+2 x_{3}^{2}-4 x_{1} x_{2}-4 x_{1} x_{3}+2 x_{2} x_{3} .
$$

We also have

$x_{1}^{2}+\left(x_{1}-x_{2}\right)^{2}+\left(x_{1}-x_{3}\right)^{2}+\left(x_{1}-x_{2}-x_{3}\right)^{2}=4 x_{1}^{2}+2 x_{2}^{2}+2 x_{3}^{2}-4 x_{1} x_{2}-4 x_{1} x_{3}+2 x_{2} x_{3}$.

Thus $C_{1}$ contains $x_{2}^{2}, x_{3}^{2}$ and also the middle ray $\left(2 x_{1}-x_{2}-x_{3}\right)^{2}+x_{2}^{2}+x_{3}^{2}$. Note that again, the middle ray is also the middle ray of the appropriate face of the matroidal cone. This computation is in fact symmetric: by appropriate change of coordinates, one can cover the monodromy cone with three matroidal cones of this type.

Remark A.7. For the $F S_{2}$ cone, the midpoint is contained in the matroidal cone

$$
\mathbb{R}_{\geq 0}\left\langle x_{1}^{2},\left(x_{1}-x_{2}\right)^{2}\right\rangle .
$$

The right half of the $F S_{2}$ cone (in Figure 7) is contained in the matroidal cone

$$
\mathbb{R}_{\geq 0}\left\langle x_{1}^{2}, x_{2}^{2},\left(x_{1}-x_{2}\right)^{2}\right\rangle
$$

(the other subcone may be studied by symmetry). We may change basis so these cones are $\mathbb{R}_{\geq 0}\left\langle y_{1}^{2}, y_{2}^{2}\right\rangle$ and $\mathbb{R}_{\geq 0}\left\langle y_{1}^{2}, y_{2}^{2},\left(y_{1}-y_{2}\right)^{2}\right\rangle$ respectively. The Delaunay decomposition of $\mathbb{R}^{2}$ for the standard cone $\sigma_{1+1}=\mathbb{R}_{\geq 0}\left\langle y_{1}^{2}, y_{2}^{2}\right\rangle$ is the decomposition into squares. The corresponding chain of toric varieties has components equal to $\mathbb{P}^{1} \times \mathbb{P}^{1}$, glued to each other along "opposite" coordinate lines $\{0\} \times \mathbb{P}^{1}$ and $\{\infty\} \times \mathbb{P}^{1}$ as well as $\mathbb{P}^{1} \times\{0\}$ and $\mathbb{P}^{1} \times\{\infty\}$. For the cone $\sigma_{K_{3}}=\mathbb{R}_{\geq 0}\left\langle y_{1}^{2}, y_{2}^{2},\left(y_{1}-y_{2}\right)^{2}\right\rangle$, the Delaunay decomposition changes: every square breaks up into two triangles. The corresponding chain of toric varieties has components equal to $\mathbb{P}^{2}$, glued to each other along their coordinate lines.

Remark A.8. For the $F S_{3}$ cone, the midpoint is contained in the matroidal cone

$$
\mathbb{R}_{\geq 0}\left\langle x_{1}^{2},\left(x_{1}-x_{2}\right)^{2},\left(x_{1}-x_{3}\right)^{2},\left(x_{1}-x_{2}-x_{3}\right)^{2}\right\rangle .
$$

The line segment joining the midpoint to the lower right corner (in Figure 7) is contained in the matroidal cone $\mathbb{R}_{\geq 0}\left\langle x_{1}^{2}, x_{2}^{2},\left(x_{1}-x_{2}\right)^{2},\left(x_{1}-x_{3}\right)^{2},\left(x_{1}-x_{2}-x_{3}\right)^{2}\right\rangle$. The right hand cone of full dimension is contained in the matroidal cone $\mathbb{R}_{\geq 0}\left\langle x_{1}^{2}, x_{2}^{2}, x_{3}^{2},\left(x_{1}-x_{2}\right)^{2}\right.$, $\left.\left(x_{1}-x_{3}\right)^{2},\left(x_{1}-x_{2}-x_{3}\right)^{2}\right\rangle$. (The other subcones may be studied by symmetry.) We may change basis so these cones are $\mathbb{R}_{\geq 0}\left\langle y_{1}^{2}, y_{2}^{2}, y_{3}^{2},\left(y_{1}-y_{2}-y_{3}\right)^{2}\right\rangle, \mathbb{R}_{\geq 0}\left\langle y_{1}^{2}, y_{2}^{2}, y_{3}^{2}\right.$, $\left.\left(y_{1}-y_{2}\right)^{2},\left(y_{1}-y_{2}-y_{3}\right)^{2}\right\rangle$, and $\mathbb{R}_{\geq 0}\left\langle y_{1}^{2}, y_{2}^{2}, y_{3}^{2},\left(y_{1}-y_{2}\right)^{2},\left(y_{1}-y_{3}\right)^{2},\left(y_{1}-y_{2}-y_{3}\right)^{2}\right\rangle$ respectively. The Delaunay decomposition of $\mathbb{R}^{3}$ with respect to the cone $\sigma_{C_{4}}=$ $\mathbb{R}_{\geq 0}\left\langle y_{1}^{2}, y_{2}^{2}, y_{3}^{2},\left(y_{1}-y_{2}-y_{3}\right)^{2}\right\rangle$ consists of the tiling by one octahedron and two tetrahedra (i.e. the cube with two tetrahedra cut from opposite corners). We direct the reader to Remark 1.4 for the Delaunay decompositions for the other cones. 


\section{Appendix B. Some examples where the Prym map extends}

In this section we consider a number of further examples of admissible covers. In short, in each of these examples, the Prym map extends to the second Voronoi, perfect cone and central cone compactifications. These examples will also be useful later when we consider degenerations of Friedman-Smith covers.

\section{B.1. Beauville examples}

A Beauville example with $n$ nodes is an admissible cover where $\widetilde{C}$ is irreducible, has exactly $n$ nodes, and all of the nodes are fixed by the involution. The dual graph has a unique vertex $\tilde{v}$ and exactly $n$ edges $\tilde{e}_{1}, \ldots, \tilde{e}_{n}$, all fixed by the involution (the dual graph in the case $n=1$ is given in Figure 3$)$. One finds that $H_{1}(\widetilde{\Gamma}, \mathbb{Z})=H_{1}(\widetilde{\Gamma}, \mathbb{Z})^{+}=$ $\mathbb{Z}\left\langle\tilde{e}_{1}, \ldots, \tilde{e}_{n}\right\rangle$. Consequently, $H_{1}(\widetilde{\Gamma}, \mathbb{Z})^{-}=0$. Thus the Prym is an abelian variety, and the Prym map extends to a neighborhood of $\widetilde{C}$. These examples lie in the $n$-fold selfintersection of $\delta_{0}^{\mathrm{ram}}$.

\section{B.2. Elementary étale examples}

An elementary étale example with $2 n$ nodes is an admissible cover where $\widetilde{C}$ is irreducible, has exactly $2 n$ nodes, and all of the nodes are exchanged pairwise by the involution. The dual graph has a unique vertex $\tilde{v}$ and exactly $2 n$ edges $\tilde{e}_{1}^{+}, \tilde{e}_{1}^{-}, \ldots, \tilde{e}_{n}^{+}, \tilde{e}_{n}^{-}$, with $\iota \tilde{e}_{i}^{+}=\tilde{e}_{i}^{-}(i=1, \ldots, n)$. The dual graph of $C$ consists of a unique vertex $v$ and $n$ edges $e_{1}, \ldots, e_{n}$, with $\tilde{e}_{i}^{ \pm}$lying over $e_{i}$ (the dual graph in the case $n=1$ is given in Figure 1$)$. In this case one has $H_{1}(\widetilde{\Gamma}, \mathbb{Z})=\mathbb{Z}\left\langle\tilde{e}_{1}^{+}, \tilde{e}_{1}^{-}, \ldots, \tilde{e}_{n}^{+}, \tilde{e}_{n}^{-}\right\rangle$. Consequently, we have $H_{1}(\widetilde{\Gamma}, \mathbb{Z})^{[-]}=\mathbb{Z}\left\langle\frac{1}{2}\left(\tilde{e}_{1}^{+}-\tilde{e}_{1}^{-}\right), \ldots, \frac{1}{2}\left(\tilde{e}_{n}^{+}-\tilde{e}_{n}^{-}\right)\right\rangle$. As $H_{1}(\widetilde{\Gamma}, \mathbb{Z})^{[-]}$is dual to $H^{1}(\widetilde{\Gamma}, \mathbb{Z})^{-}$, we can see that if we set $z_{i}=\frac{1}{2}\left(\tilde{e}_{i}^{+}-\tilde{e}_{i}^{-}\right)$for $i=1, \ldots, n$, then we may take $\ell_{e_{i}}=z_{i}^{\vee}$ $(i=1, \ldots, n)$. The matrix expressing the $\ell_{e_{i}}$ in terms of this basis is then the identity, so clearly (V) of Theorem 5.6 holds. In addition, we can take the standard positive definite form $z_{1}^{2}+\cdots+z_{n}^{2}$ to show that $(\mathrm{P})$ and $(\mathrm{C})$ of Theorem 5.6 both hold. In conclusion, the Prym map extends to the second Voronoi, perfect cone, and central cone compactifications in a neighborhood of an elementary étale example. These examples lie in the $n$-fold self-intersection of $\delta_{0}^{\prime}$.

Remark B.1. The monodromy cone is the standard cone $\sigma_{1+\cdots+1}=\mathbb{R}_{\geq 0}\left\langle x_{1}^{2}, \ldots, x_{n}^{2}\right\rangle$. The associated Delaunay decomposition of $\mathbb{R}^{n}$ is the tiling by $n$-cubes. The associated chain of toric varieties has components equal to $\left(\mathbb{P}^{1}\right)^{n}$ with "opposite sides" glued.

\section{B.3. Wirtinger examples $\left(W_{n}\right)$}

A Wirtinger example with $2 n$ nodes is an admissible double cover where $\widetilde{C}$ has exactly two irreducible components, which are interchanged by the involution, and $\widetilde{C}$ has exactly $2 n$ nodes, all of which join the two components, and are interchanged pairwise by the involution. The dual graph $\widetilde{\Gamma}$ of $\widetilde{C}$ has vertices $V(\widetilde{\Gamma})=\left\{\tilde{v}^{+}, \tilde{v}^{-}\right\}$and edges $E(\widetilde{\Gamma})=$ 
$\left\{\tilde{e}_{1}^{+}, \tilde{e}_{1}^{-}, \ldots, \tilde{e}_{n}^{+}, \tilde{e}_{n}^{-}\right\}$; we orient $\tilde{e}_{i}^{+}$from $\tilde{v}^{-}$to $\tilde{v}^{+}$, and $\tilde{e}_{i}^{-}$in the opposite direction. The involution $\iota$ acts by $\iota\left(\tilde{v}^{+}\right)=\tilde{v}^{-}$and $\iota\left(\tilde{e}_{i}^{+}\right)=\tilde{e}_{i}^{-}(i=1, \ldots, n)$. The dual graph of $C$ consists of a single vertex $v$ and $n$ edges $e_{1}, \ldots, e_{n}$ with $\tilde{e}_{i}^{ \pm}$lying over $e_{i}$.

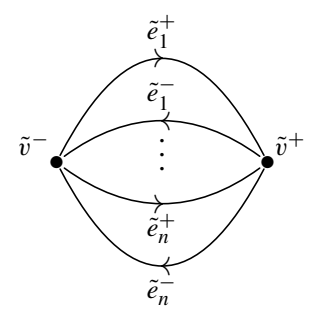

Fig. 8. Dual graph of a Wirtinger example with $2 n \geq 2 \operatorname{nodes}\left(W_{n}\right)$.

One has $H_{1}(\widetilde{\Gamma}, \mathbb{Z})=\mathbb{Z}\left\langle\tilde{e}_{1}^{+}+\tilde{e}_{1}^{-}, \ldots, \tilde{e}_{n}^{+}+\tilde{e}_{n}^{-}, \tilde{e}_{1}^{+}+\tilde{e}_{2}^{-}, \ldots, \tilde{e}_{n-1}^{+}+\tilde{e}_{n}^{-}\right\rangle$so that in turn $H_{1}(\widetilde{\Gamma}, \mathbb{Z})^{[-]} \cong \mathbb{Z}\left(\frac{1}{2}\left(\tilde{e}_{1}^{+}-\tilde{e}_{1}^{-}\right)-\frac{1}{2}\left(\tilde{e}_{2}^{+}-\tilde{e}_{2}^{-}\right), \ldots, \frac{1}{2}\left(\tilde{e}_{n-1}^{+}-\tilde{e}_{n-1}^{-}\right)-\frac{1}{2}\left(\tilde{e}_{n}^{+}-\tilde{e}_{n}^{-}\right)\right\rangle$. For brevity, set

$$
z_{1}=\frac{1}{2}\left(\tilde{e}_{1}^{+}-\tilde{e}_{1}^{-}\right)-\frac{1}{2}\left(\tilde{e}_{2}^{+}-\tilde{e}_{2}^{-}\right), \ldots, z_{n-1}=\frac{1}{2}\left(\tilde{e}_{n-1}^{+}-\tilde{e}_{n-1}^{-}\right)-\frac{1}{2}\left(\tilde{e}_{n}^{+}-\tilde{e}_{n}^{-}\right)
$$

so that $H_{1}(\widetilde{\Gamma}, \mathbb{Z})^{[-]} \cong \mathbb{Z}\left\langle z_{1}, \ldots, z_{n-1}\right\rangle$. Then

$$
H^{1}(\widetilde{\Gamma}, \mathbb{Z})^{-}=\left(H_{1}(\widetilde{\Gamma}, \mathbb{Z})^{[-]}\right)^{\vee} \cong \mathbb{Z}\left\langle z_{1}^{\vee}, \ldots, z_{n-1}^{\vee}\right\rangle .
$$

One can check that for $i=1, \ldots, n$, one may take $\ell_{e_{i}}=\left(\tilde{e}_{i}^{+}\right)^{\vee}-\left(\tilde{e}_{i}^{-}\right)^{\vee}$. The $n \times(n-1)$ matrix expressing the $\ell_{e_{i}}$ in terms of the basis $z_{1}^{\vee}, \ldots, z_{n-1}^{\vee}$ is

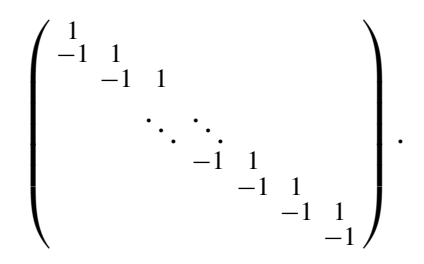

Considering the $(n-1) \times(n-1)$ minors, we see that $(\mathrm{V})$ holds. Now consider the quadratic form

$$
z_{1}^{2}+z_{1} z_{2}+z_{2}^{2}+\cdots+z_{n-2}^{2}+z_{n-2} z_{n-1}+z_{n-1}^{2} .
$$

The associated matrix has entries equal to 1 on the diagonal, and to $1 / 2$ above and below the diagonal. The determinant of such a square matrix of size $m$ is $(m+1) / 2^{m}$. Consequently, the quadratic form is positive definite, since all of its leading minors are positive. It is easy to see by inspection that the rows of the matrix (B.1) are exactly the shortest vectors of this form. Hence the monodromy cone is a matroidal cone which also satisfies (C). In fact, this cone is contained in the $A_{n-1}$-cone (see (1.5)). In conclusion, the Prym map extends to the second Voronoi, perfect cone, and central cone compactifications in a neighborhood of a Wirtinger example. 
Remark B.2. The $W_{n}$ monodromy cone is $\mathbb{R}_{\geq 0}\left\langle x_{1}^{2},\left(x_{1}-x_{2}\right)^{2}, \ldots,\left(x_{n-2}-x_{n-1}\right)^{2}, x_{n-1}^{2}\right\rangle$. This is equivalent to the cone $\mathbb{R}_{\geq 0}\left\langle\left(y_{1}+\cdots+y_{n-1}\right)^{2}, y_{1}^{2}, \ldots, y_{n-1}^{2}\right\rangle$. For $n=3$, the Delaunay decomposition of $\mathbb{R}^{2}$ is the tiling by 2 -simplices obtained by slicing the standard square into two triangles. The associated chain of toric varieties has components equal to $\mathbb{P}^{2}$, glued along coordinate hyperplanes. We have already seen in Remark A.8 that for $n=4$, the Delaunay decomposition of $\mathbb{R}^{3}$ with respect to the cone $\sigma_{C_{4}}=\mathbb{R}_{\geq 0}\left\langle y_{1}^{2}, y_{2}^{2}, y_{3}^{2}\right.$, $\left.\left(y_{1}-y_{2}-y_{3}\right)^{2}\right\rangle$ consists of the tiling by one octahedron and two tetrahedra (i.e. the cube with two tetrahedra cut from opposite corners).

\section{B.4. Mixed Beauville-étale examples}

In these examples we consider admissible double covers where $\tilde{C}$ has a dual graph with a single vertex $\tilde{v}$, and $2 n+m$ edges

$$
\tilde{e}_{1}^{+}, \tilde{e}_{1}^{-}, \ldots, \tilde{e}_{n}^{+}, \tilde{e}_{n}^{-}, \tilde{e}_{2 n+1}, \ldots, \tilde{e}_{2 n+m},
$$

where $\iota \tilde{e}_{i}^{+}=\tilde{e}_{i}^{-}(i=1, \ldots, n)$ and $\iota \tilde{e}_{j}=\tilde{e}_{j}(j=2 n+1, \ldots, 2 n+m)$. Then $H_{1}(\widetilde{\Gamma}, \mathbb{Z})=$ $\mathbb{Z}\left\langle\tilde{e}_{1}^{+}, \tilde{e}_{1}^{-}, \ldots, \tilde{e}_{n}^{+}, \tilde{e}_{n}^{-}, \tilde{e}_{2 n+1}, \ldots, \tilde{e}_{2 n+m}\right\rangle$, and

$$
H_{1}(\widetilde{\Gamma}, \mathbb{Z})^{[-]}=\mathbb{Z}\left\langle\frac{1}{2}\left(\tilde{e}_{1}^{+}-\tilde{e}_{1}^{-}\right), \ldots, \frac{1}{2}\left(\tilde{e}_{n}^{+}-\tilde{e}_{n}^{-}\right)\right\rangle .
$$

One can then check that $\ell_{e_{i}}=\left(\tilde{e}_{i}^{+}\right)^{\vee}-\left(\tilde{e}_{i}^{-}\right)^{\vee}$ for $i=1, \ldots, n$ and $\ell_{e_{j}}=0$ for $j=$ $2 n+1, \ldots, 2 n+m$. In other words, we are exactly back in the situation of the elementary étale examples, and consequently the Prym map extends.

\section{B.5. Mixed Beauville-Friedman-Smith examples}

In these examples we consider admissible covers where $\tilde{C}$ has a dual graph with two vertices $\tilde{v}_{1}, \tilde{v}_{2}$, fixed by the involution, and $2 n+m$ edges

$$
\tilde{e}_{1}^{+}, \tilde{e}_{1}^{-}, \ldots, \tilde{e}_{n}^{+}, \tilde{e}_{n}^{-}, \tilde{e}_{2 n+1}, \ldots, \tilde{e}_{2 n+m},
$$

where $\iota \tilde{e}_{i}^{+}=\tilde{e}_{i}^{-}(i=1, \ldots, n)$ and $i \tilde{e}_{j}=\tilde{e}_{j}(j=2 n+1, \ldots, 2 n+m)$. One finds that $H_{1}(\widetilde{\Gamma}, \mathbb{Z})^{[-]}=\mathbb{Z}\left\langle\frac{1}{2}\left(\tilde{e}_{1}^{+}-\tilde{e}_{1}^{-}\right), \ldots, \frac{1}{2}\left(\tilde{e}_{n}^{+}-\tilde{e}_{n}^{-}\right)\right\rangle, \ell_{e_{i}}=\left(\tilde{e}_{i}^{+}\right)^{\vee}-\left(\tilde{e}_{i}^{-}\right)^{\vee}$ for $i=1, \ldots, n$, and $\ell_{e_{j}}=0$ for $j=2 n+1, \ldots, 2 n+m$. Consequently, we find ourselves exactly back in the case of the elementary étale examples, and the Prym map extends in a neighborhood of these examples.

\section{Appendix C. Degenerations of Friedman-Smith covers}

Here we investigate several classes of degenerations of Friedman-Smith covers that arise in describing the resolution of the Prym map in low genus. 


\section{C.1. Vologodsky's degenerations $\left(D R_{n}\right)$}

In [Vol04], Vologodsky investigates a certain class of degenerations of Friedman-Smith covers, which he denotes by $D R_{n}$. These are covers with $n$ smooth irreducible components $\widetilde{C}_{1}, \ldots, \widetilde{C}_{n}$, all preserved by the involution, with each irreducible component $\widetilde{C}_{i}$ meeting $\widetilde{C}_{i-1}$ and $\widetilde{C}_{i+1}$ in exactly two nodes each, which are interchanged pairwise by the involution. Here $\widetilde{C}_{0}=\widetilde{C}_{n}$ and $\widetilde{C}_{n+1}=\widetilde{C}_{1}$.

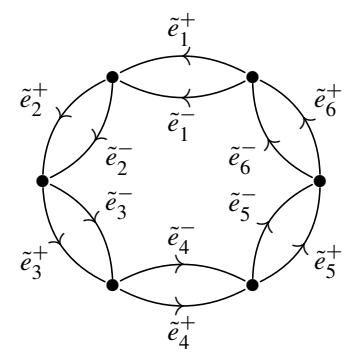

Fig. 9. Dual graph of a $D R_{6}$ cover.

The $D R_{n}$ dual graph is similar to the one in Figure 9, except that it has $2 n$ edges. One can see that $D R_{1}$ lies in $\delta_{0}^{\prime}, D R_{2}=F S_{2}$, and that for $n \geq 2, D R_{n}$ is contained in $\overline{F S}_{2}$ as well as as in the $n$-fold self-intersection of $\delta_{0}^{\prime}$. Moreover, for $n \geq 2, D R_{n+1} \subseteq \overline{D R}_{n}$. Étale locally near a $D R_{n}$ example with $n \geq 2$, each of the $\left(\begin{array}{l}n \\ 2\end{array}\right)$ intersections of two étale local components of the $\delta_{0}^{\prime}$ divisors is an étale local component of the $\overline{F S}_{2}$ locus.

One can check that

$$
H_{1}(\widetilde{\Gamma}, \mathbb{Z})=\mathbb{Z}\left\langle\tilde{e}_{1}^{+}-\tilde{e}_{1}^{-}, \ldots, \tilde{e}_{n}^{+}-\tilde{e}_{n}^{-}, \tilde{e}_{1}^{+}+\cdots+\tilde{e}_{n}^{+}\right\rangle
$$

and

$$
H_{1}(\widetilde{\Gamma}, \mathbb{Z})^{[-]}=\mathbb{Z}\left|\tilde{e}_{1}^{+}-\tilde{e}_{1}^{-}, \ldots, \tilde{e}_{n-1}^{+}-\tilde{e}_{n-1}^{-}, \frac{1}{2}\left(\tilde{e}_{1}^{+}-\tilde{e}_{1}^{-}\right)+\cdots+\frac{1}{2}\left(\tilde{e}_{n}^{+}-\tilde{e}_{n}^{-}\right)\right\rangle .
$$

The monodromy cone is then given by the $n \times n$ matrix

$$
D R_{n} \quad\left(\begin{array}{ccc|cc}
2 & 0 & \cdots & 0 & 1 \\
0 & 2 & 0 & 0 & 1 \\
0 & 0 & 2 & 0 & 1 \\
\hline & & 0 & 2 & 1
\end{array}\right)=\left(\begin{array}{cc|cc}
2 \mathrm{Id}_{n-2} & 0 & 1 \\
\hline 0 & F S_{2}
\end{array}\right) .
$$

One can see immediately that these cones are simplicial. Vologodsky [Vol04, Prop. 1.3] gives the second Voronoi decomposition of these cones: If we view the cone as a cone over the standard $n-1$ simplex in $\mathbb{R}^{n}$, the second Voronoi decomposition is the collection of cones defined by the hyperplanes in $\mathbb{R}^{n}$ defined by $\sum_{j \in J} c_{j}=\sum_{j \notin J} c_{j}$ for every proper subset $J \subset\{1, \ldots, n\}$. For $n=2,3$ one obtains the decompositions depicted in Figure 10. For dimension reasons, this also gives the perfect and central cone decomposition. 


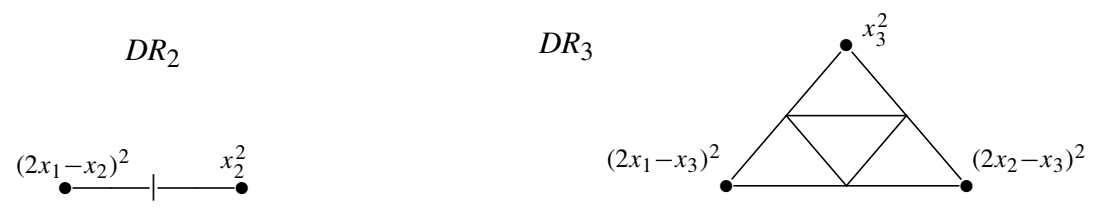

Fig. 10. Decomposition of the monodromy cone for $D R_{2}$ and $D R_{3}$ covers.

We now describe the perfect cone decomposition of the monodromy cone of $D R_{n}$ covers for all $n$. Before doing this, let us introduce some notation. Let $\Delta^{n}$ be the standard $n$-simplex in $\mathbb{R}^{n+1}$, i.e. the convex hull of the basis vectors $e_{1}, \ldots, e_{n+1}$. Define $\Delta_{i}^{n}$ to be the convex hull of the $n+1$ vectors

$$
\frac{e_{i}+e_{1}}{2}, \ldots, \frac{e_{i}+e_{n+1}}{2}
$$

In other words, $\Delta_{i}^{n}$ is the $n$-simplex obtained by cutting off the $i$-th corner of the original simplex at the midpoint of the edges containing $e_{i}$.

Proposition C.1. If we view the monodromy cone for a $D R_{n}$ degeneration as cone over the $n$-simplex $\Delta^{n}$, the perfect cone decomposition is given by

$$
\Delta^{n}=\Delta_{1}^{n} \cup \cdots \cup \Delta_{n}^{n} \cup \overline{\Delta^{n}-\bigcup_{i=1}^{n} \Delta_{i}^{n}} .
$$

Moreover, the cones over the $\Delta_{i}^{n}$ are all contained in matroidal cones of type A, and the remaining cone, over $\overline{\Delta^{n}-\bigcup_{i=1}^{n} \Delta_{i}^{n}}$, is contained in a perfect cone of type $D$ (see Remark 1.9). In particular, the second Voronoi decomposition of a $D R_{n}$ monodromy cone is a refinement of the perfect cone decomposition.

Remark C.2. This also gives the decomposition of the monodromy cone into cones contained in cones in the central cone decomposition (CCD).

Proof of Proposition C.1. From (C.1), and an elementary change of coordinates, the $D R_{n}$ monodromy cone can be taken to be generated by the $n$ quadratic forms $M_{1}=x_{1}^{2}, M_{i}=$ $\left(2 x_{i}-x_{1}\right)^{2}(i=2, \ldots, n)$; note that in fact with these definitions $M_{i}=\left(2 x_{i}-x_{1}\right)^{2}$ for all $i$. Now define

$$
N_{a b}:=\frac{1}{2}\left(M_{a}+M_{b}\right), \quad 1 \leq a \leq b \leq n,
$$

to be the midpoint of the segment joining $M_{a}$ to $M_{b}$. We claim that the cones

$$
\Delta_{i}^{n}:=\mathbb{R}_{\geq 0}\left\langle N_{i, 1}, \ldots, N_{i, n}\right\rangle, \quad D:=\mathbb{R}_{\geq 0}\left\langle N_{i j}\right\rangle_{i \neq j}
$$

are contained in cones in the perfect cone decomposition, of types $A$ and $D$ respectively.

To see this, observe that

$$
\left.N_{i j}=\frac{1}{2}\left(2 x_{i}-x_{1}\right)^{2}+\left(2 x_{j}-x_{1}\right)^{2}\right)=\left(x_{1}-x_{i}-x_{j}\right)^{2}+\left(x_{i}-x_{j}\right)^{2},
$$


which reduces in the case $i=1$ to

$$
N_{1 i}=\left(x_{1}-x_{i}\right)^{2}+x_{i}^{2}
$$

Now one can easily check that $\Delta_{1}^{n}=\mathbb{R}_{\geq 0}\left\langle N_{1,1}, \ldots, N_{1, n}\right\rangle$ is contained in a cone of type $A$ (see (1.5)). The other cones $\Delta_{2}^{n}, \ldots, \Delta_{n}^{n}$ are contained in type $A$ cones by symmetry. Similarly, one can check that the cone $D$ is contained in a cone of type $D$ (see (1.5)).

Remark C.3. As mentioned above, the geometric description near a $D R_{n}$ example is that there are $n$ copies of $\delta_{0}^{\prime}$ meeting. The $\left(\begin{array}{l}n \\ 2\end{array}\right) 2$-fold intersections correspond to $\overline{F S}_{2}$ loci. The toric resolution given by the above decomposition of the monodromy cone is supported along the union of these loci. For $n=2$ it is the blow-up of the $\overline{F S}_{2}$ locus. For $n=3$ it is a more complicated birational modification described in more detail in $\S 8$.

\section{C.2. Friedman-Smith-Wirtinger degenerations $\left(F S_{n}+W_{m}\right)$}

In these examples, we degenerate one of the curves in a Friedman-Smith cover $\left(F S_{n}\right)$ to a Wirtinger example $\left(W_{m}\right)$. The dual graph is given in Figure 11 .

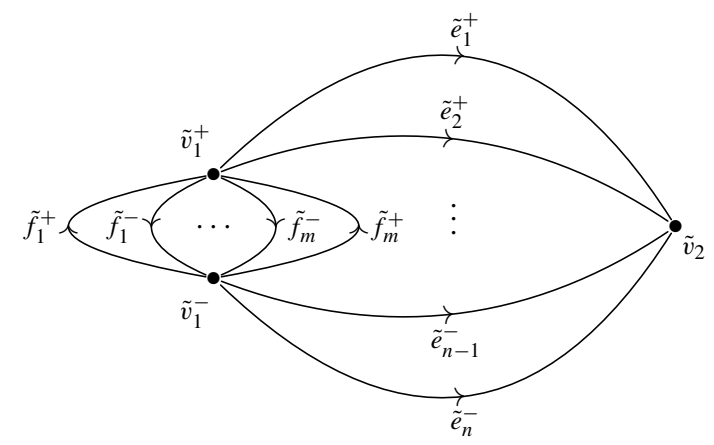

Fig. 11. Dual graph of an $F S_{n}+W_{m}$ degeneration of a Friedman-Smith cover with $2 n \geq 2$ nodes.

These examples lie in the $(n+m)$-fold self-intersection of $\delta_{0}^{\prime}$. They lie in $\overline{F S}_{2} \cup \overline{F S}_{3}$ if and only if $n=2,3$. One can check that

$$
\begin{aligned}
H_{1}(\widetilde{\Gamma}, \mathbb{Z})= & \mathbb{Z}\left\langle\left(\tilde{e}_{1}^{+}-\tilde{e}_{1}^{-}\right)+\tilde{f}_{1}^{+}, \ldots,\left(\tilde{e}_{n}^{+}-\tilde{e}_{n}^{-}\right)+\tilde{f}_{1}^{+},\left(\tilde{e}_{1}^{+}-\tilde{e}_{2}^{-}\right)+\tilde{f}_{1}^{+}, \ldots,\right. \\
& \left.\left(\tilde{e}_{n-1}^{+}-\tilde{e}_{n}^{-}\right)+\tilde{f}_{1}^{+}, \tilde{f}_{1}^{+}-\tilde{f}_{1}^{-}, \ldots, \tilde{f}_{m}^{+}-\tilde{f}_{m}^{-}, \tilde{f}_{1}^{+}-\tilde{f}_{2}^{-}, \ldots, \tilde{f}_{m-1}^{+}-\tilde{f}_{m}^{-}\right\rangle
\end{aligned}
$$

and

$$
\begin{aligned}
& H_{1}(\widetilde{\Gamma}, \mathbb{Z})^{[-]}=\mathbb{Z}\left\langle\left(\tilde{e}_{1}^{+}-\tilde{e}_{1}^{-}\right)+\frac{1}{2}\left(\tilde{f}_{1}^{+}-\tilde{f}_{1}^{-}\right), \frac{1}{2}\left(\tilde{e}_{1}^{+}-\tilde{e}_{1}^{-}\right)+\frac{1}{2}\left(\tilde{e}_{2}^{+}-\tilde{e}_{2}^{-}\right)+\frac{1}{2}\left(\tilde{f}_{1}^{+}-\tilde{f}_{1}^{-}\right),\right. \\
& \ldots, \frac{1}{2}\left(\tilde{e}_{n-1}^{+}-\tilde{e}_{n-1}^{-}\right)+\frac{1}{2}\left(\tilde{e}_{n}^{+}-\tilde{e}_{n}^{-}\right)+\frac{1}{2}\left(\tilde{f}_{1}^{+}-\tilde{f}_{1}^{-}\right), \\
&\left.\frac{1}{2}\left(\tilde{f}_{1}^{+}-\tilde{f}_{1}^{-}\right)-\frac{1}{2}\left(\tilde{f}_{2}^{+}-\tilde{f}_{2}^{-}\right), \ldots, \frac{1}{2}\left(\tilde{f}_{m-1}^{+}-\tilde{f}_{m-1}^{-}\right)-\frac{1}{2}\left(\tilde{f}_{m}^{+}-\tilde{f}_{m}^{-}\right)\right\rangle .
\end{aligned}
$$


One can then show that the monodromy cone is generated by the squares of the rows of the matrix

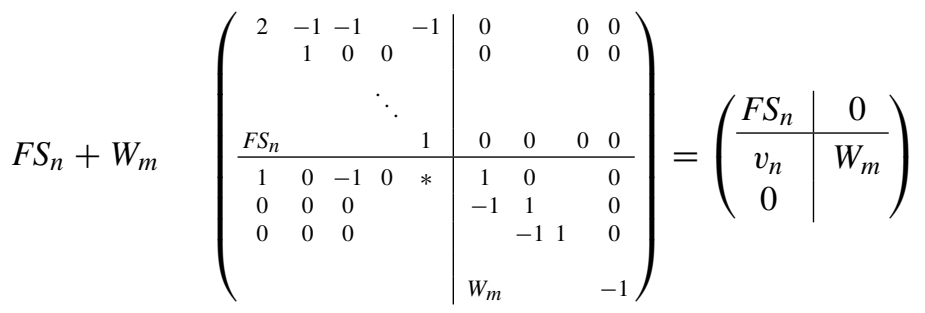

where $*$ is defined to be -1 or 0 depending on whether $n$ is odd or even respectively. Recall that the $W_{m}$ matrix is an $m \times(m-1)$ matrix (see $\S$ B.3). In particular, for $m=1$ the two right hand blocks of (C.2) are missing.

Lemma C.4. Consider the case $m=1$. The monodromy cone for an $F S_{n}+W_{1}$ degeneration is not contained in a cone in the PCD for $n=2,3$. The monodromy cone is contained in a cone in the PCD for $n=1,4,5,6,7$.

Remark C.5. In fact the monodromy cone is also contained in a cone in the CCD for $n=1,4,5,7$.

Proof of Lemma C.4. For $n=1$, this is clear. For $n=2,3$, we have already observed above that these examples are degenerations of $\overline{F S}_{2}$ and $\overline{F S}_{3}$ examples, and so are not contained in cones in the PCD. For $n=4$, the quadratic form $Q$ defined in (A.2) shows that the monodromy cone is contained in a cone in the PCD. For $n=5,6,7$, the quadratic forms

$$
\begin{aligned}
& Q_{5}=\left(\begin{array}{ccccc}
1 & 1 / 2 & 1 / 2 & 1 / 2 & 1 / 2 \\
1 / 2 & 1 & 0 & 0 & 1 / 2 \\
1 / 2 & 0 & 1 & 0 & 0 \\
1 / 2 & 0 & 0 & 1 & 0 \\
1 / 2 & 1 / 2 & 0 & 0 & 1
\end{array}\right), \quad Q_{6}=1 / 20\left(\begin{array}{cccccc}
23 & 7 & 9 & 7 & 9 & 7 \\
7 & 15 & 1 & -1 & 1 & -1 \\
9 & 1 & 15 & 1 & -1 & 1 \\
7 & -1 & 1 & 15 & 1 & -1 \\
9 & 1 & -1 & 1 & 15 & 1 \\
7 & -1 & 1 & -1 & 1 & 15
\end{array}\right) \\
& Q_{7}=\left(\begin{array}{ccccccc}
1 & 1 / 2 & -1 / 2 & 1 / 2 & 1 / 2 & 1 / 2 & 1 / 2 \\
1 / 2 & 1 & 0 & 0 & 0 & 0 & 0 \\
-1 / 2 & 0 & 1 & -1 / 2 & -1 / 2 & -1 / 2 & -1 / 2 \\
1 / 2 & 0 & -1 / 2 & 1 & 1 / 2 & 1 / 2 & 0 \\
1 / 2 & 0 & -1 / 2 & 1 / 2 & 1 & 1 / 2 & 0 \\
1 / 2 & 0 & -1 / 2 & 1 / 2 & 1 / 2 & 1 & 0 \\
1 / 2 & 0 & -1 / 2 & 0 & 0 & 0 & 1
\end{array}\right)
\end{aligned}
$$

show that the monodromy cone is contained in a cone in the PCD. We thank Mathieu Dutour Sikiric for providing us with the metric $Q_{6}$, which actually shows that the monodromy cone is a cone in the PCD.

We now turn our attention to the perfect cone decomposition of the monodromy cone for $n=2,3, m=1$, that is, for the cones

$$
F S_{2}+W_{1} \quad\left(\begin{array}{cc}
2 & -1 \\
0 & 1 \\
\hline 1 & 0
\end{array}\right) \text { and } F S_{3}+W_{1}\left(\begin{array}{ccc}
2 & -1 & -1 \\
0 & 1 & 0 \\
0 & 0 & 1 \\
\hline 1 & 0 & -1
\end{array}\right)
$$


It turns out that the analysis in the proof of Lemma A.5 actually provides the decomposition in this case as well. For $n=2$, the star subdivision associated to one of the edges of the cone provides the decomposition (see Figure 12). The dashed lines in the figure show

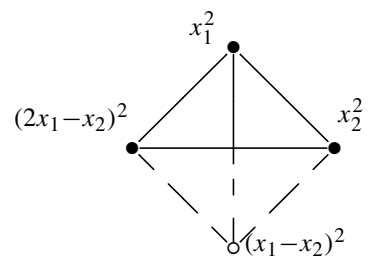

Fig. 12. Perfect cone decomposition of an $F S_{2}+W_{1}$ monodromy cone.

the ambient matroidal cones giving the decomposition of the monodromy cone (which are depicted by the solid lines).

For $n=3$, the star subdivision associated to one of the 2-dimensional faces of the cone provides the decomposition; see Figure 13. The open bullet in the figure is the

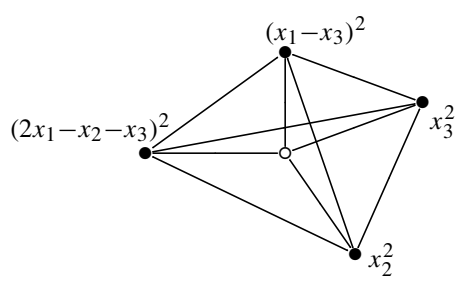

Fig. 13. Perfect cone decomposition of an $F S_{3}+W_{1}$ monodromy cone.

midpoint of the bottom face, i.e. it corresponds to the quadratic form $\frac{1}{3}\left(\left(2 x_{1}-x_{2}-x_{3}\right)^{2}\right.$ $\left.+x_{2}^{2}+x_{3}^{2}\right)$. Note that the face $C\left(x_{2}^{2}, x_{3}^{2},\left(x_{1}-x_{3}\right)^{2}\right)$ is contained in the cone

$$
C_{1}=\mathbb{R}_{\geq 0}\left\langle x_{1}^{2}, x_{2}^{2}, x_{3}^{2},\left(x_{1}-x_{2}\right)^{2},\left(x_{1}-x_{3}\right)^{2},\left(x_{1}-x_{2}-x_{3}\right)^{2}\right\rangle,
$$

discussed in the proof of Lemma A.5. We showed there that the cone $C_{1}$ contains the midpoint of the bottom face (the ray generated by $\left(2 x_{1}-x_{2}-x_{3}\right)^{2}+x_{2}^{2}+x_{3}^{2}$, corresponding to the bullet point in the diagram). The other subcones can be obtained by change of coordinates.

Remark C.6. Geometrically, we obtain the following picture of the resolution. For the case $n=2$, there are three copies of $\delta_{0}^{\prime}$ meeting; exactly one pair of intersections gives rise to a single $\overline{F S}_{2}$ locus. This locus is blown up. For the $n=3$ case, there is a single 3 -fold intersection of the $\delta_{0}^{\prime}$ divisors that gives an $\overline{F S}_{3}$ locus. This is blown up.

\section{C.3. Friedman-Smith-Friedman-Smith degenerations $\left(F S_{n_{1}+n_{2}}+F S_{m}\right)$}

Here we consider the case where one of the curves in a Friedman-Smith cover of type $F S_{n}$ degenerates to a Friedman-Smith cover of type $F S_{m}$. It is best to break $n$ down into $n=n_{1}+n_{2}$. The dual graph is depicted in Figure 14 . 


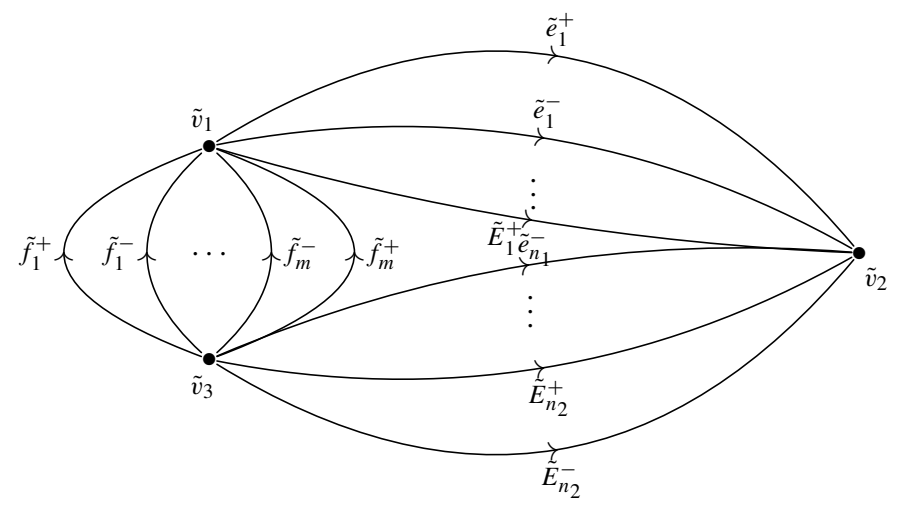

Fig. 14. Dual graph of $F S_{n_{1}+n_{2}}+F S_{m}$ degeneration of a Friedman-Smith example with $2 n=$ $2\left(n_{1}+n_{2}\right) \geq 2$ nodes.

Geometrically, we have $n+m$ copies of $\delta_{0}^{\prime}$ meeting. An $F S_{n_{1}+n_{2}}+F S_{m}$ degeneration is a degeneration of an $F S_{2}$ or $F S_{3}$ example if and only if one (or more) of $m+n_{1}, m+n_{2}$ or $n_{1}+n_{2}$ is equal to 2 or 3 . This is also a $D R_{3}$ example if $n_{1}=n_{2}=m=1$.

The case where $n_{1}, n_{2}$ or $m$ is 0 is elementary (the monodromy matrix can be made block-diagonal), so we will ignore this in the analysis that follows. One can show that

$$
\begin{array}{r}
H_{1}(\widetilde{\Gamma}, \mathbb{Z})=\mathbb{Z}\left\langle\tilde{e}_{1}^{+}-\tilde{e}_{1}^{-}, \ldots, \tilde{e}_{n_{1}}^{+}-\tilde{e}_{n_{1}}^{-}, \tilde{E}_{1}^{+}-\tilde{E}_{1}^{-}, \ldots, \tilde{E}_{n_{2}}^{+}-\tilde{E}_{n_{2}}^{-},\right. \\
\tilde{e}_{1}^{+}-\tilde{e}_{2}^{-}, \ldots, \tilde{e}_{n_{1}-1}^{+}-\tilde{e}_{n_{1}}^{-}, \tilde{e}_{n_{1}}^{+}-\tilde{E}_{1}^{-}+\tilde{f}_{1}^{+}, \tilde{E}_{1}^{+}-\tilde{E}_{2}^{-}, \ldots, \tilde{E}_{n_{2}-1}^{+}-\tilde{E}_{n_{2}}^{-}, \\
\left.\tilde{f}_{1}^{+}-\tilde{f}_{1}^{-}, \ldots, \tilde{f}_{m}^{+}-\tilde{f}_{m}^{-}, \tilde{f}_{1}^{+}-\tilde{f}_{2}^{-}, \ldots, \tilde{f}_{m-1}^{+}-\tilde{f}_{m}^{-}\right\rangle
\end{array}
$$

and

$$
\begin{array}{r}
H_{1}(\widetilde{\Gamma}, \mathbb{Z})^{[-]}=\mathbb{Z}\left(\tilde{e}_{1}^{+}-\tilde{e}_{1}^{-}, \frac{1}{2}\left(\tilde{e}_{1}^{+}-\tilde{e}_{1}^{-}\right)+\frac{1}{2}\left(\tilde{e}_{2}^{+}-\tilde{e}_{2}^{-}\right), \ldots, \frac{1}{2}\left(\tilde{e}_{n_{1}-1}^{+}-\tilde{e}_{n_{1}-1}^{-}\right)+\frac{1}{2}\left(\tilde{e}_{n_{1}}^{+}-\tilde{e}_{n_{1}}^{-}\right),\right. \\
\quad \frac{1}{2}\left(\tilde{E}_{1}^{+}-\tilde{E}_{1}^{-}\right)+\frac{1}{2}\left(\tilde{E}_{2}^{+}-\tilde{E}_{2}^{-}\right), \ldots, \frac{1}{2}\left(\tilde{E}_{n_{2}-1}^{+}-\tilde{E}_{n_{2}-1}^{-}\right)+\frac{1}{2}\left(\tilde{E}_{n_{2}}^{+}-\tilde{E}_{n_{2}}^{-}\right), \\
\left.\left(\tilde{f}_{1}^{+}-\tilde{f}_{1}^{-}\right), \frac{1}{2}\left(\tilde{f}_{1}^{+}-\tilde{f}_{1}^{-}\right)+\frac{1}{2}\left(\tilde{f}_{2}^{+}-\tilde{f}_{2}^{-}\right), \ldots, \frac{1}{2}\left(\tilde{f}_{m-1}^{+}-\tilde{f}_{m-1}^{-}\right)+\frac{1}{2}\left(\tilde{f}_{m}^{+}-\tilde{f}_{m}^{-}\right)\right\rangle .
\end{array}
$$

The matrix for the monodromy can then be put in the form

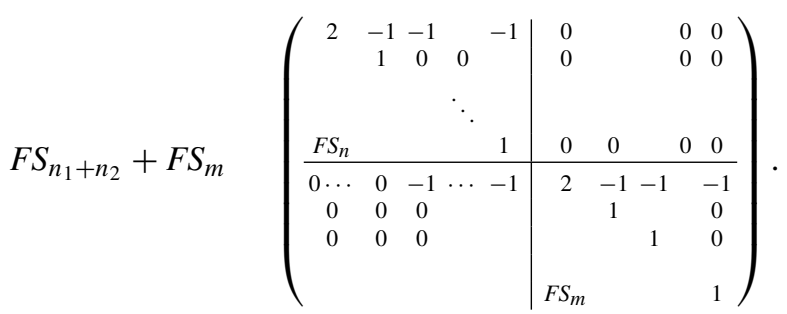


The matrix is almost block-diagonal, with the $F S_{n}$ and $F S_{m}$ matrices. The $(n+1)$-st row starts with $n_{1}$ zeros, then has $n_{2}$ negative ones, and has for its last $m$ entries the first row of the $F S_{m}$ matrix.

Lemma C.7. The monodromy cone for an $F S_{n_{1}+n_{2}}+F S_{n}$ degeneration is contained in a cone in the PCD if and only if the cover is not a degeneration of an $F_{2}$ or $F S_{3}$ example (i.e. if and only if $m+n_{1}, m+n_{2}, n_{1}+n_{2} \neq 2,3$ ).

Proof. This is essentially identical to the proof of Proposition A.3, and is left to the reader.

Remark C.8. If all of the sums $n_{1}+n_{2}, n_{1}+m, n_{2}+m$ are divisible by 4 , then the monodromy cone is contained in a cone in the central cone decomposition.

Remark C.9. For the case $n_{1}=n_{2}=m=1$, it was observed above that this is also a $D R_{3}$ cone. In particular, we have already worked out the decomposition into perfect (in fact matroidal) cones. The other cases where $F S_{n_{1}+n_{2}}+F S_{m}$ degenerations are degenerations of $F S_{2}$ or $F S_{3}$ covers satisfy the condition that one of $n_{1}, n_{2}, m$ is 0 and so the decompositions can be described in terms of Friedman-Smith examples (see §6).

Specifically, let us consider the cone decomposition for $F S_{2+0}+F S_{1}$. The matrix for the monodromy cone can be put in the form

$$
\left(\begin{array}{ccc}
2 & -1 & 0 \\
0 & 1 & 0 \\
0 & 0 & 1
\end{array}\right)
$$

Then one can easily check that the matrices

$$
\left(\begin{array}{ccc}
2 & -1 & 0 \\
0 & 0 & 1 \\
1 & -1 & 0
\end{array}\right), \quad\left(\begin{array}{ccc}
1 & -1 & 0 \\
0 & 1 & 0 \\
0 & 0 & 1
\end{array}\right)
$$

are matroidal. In short, the perfect cone decomposition is given in Figure 15. The dashed lines in the figure show the ambient matroidal cones giving the decomposition of the monodromy cone (which are depicted by the solid lines).

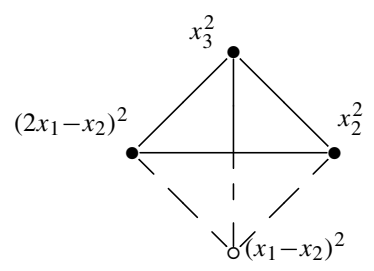

Fig. 15. Perfect cone decomposition of an $F S_{2+0}+F S_{1}$ monodromy cone. 


\section{C.4. Friedman-Smith- $\delta_{i}$ degenerations}

Here we consider the case where one of the curves in a Friedman-Smith cover degenerates to a generic cover in $\delta_{i}$ (or $\delta_{g+1-i}$ ). We call these $F S_{n_{1}+n_{2}}+\delta_{i}$ degenerations.

Geometrically, this is the $(n+1)$-fold intersection of $\delta_{0}^{\prime}$ (unless $n_{1}=0$, in which case it is the $n$-fold intersection of $\delta_{0}^{\prime}$ with $\delta_{i}\left(\right.$ or $\left.\left.\delta_{g+1-i}\right)\right)$. This is a degeneration of an $F S_{2}$ or $F S_{3}$ cover if and only if $n_{1}+n_{2}=2,3$ or $n_{2}+1=2,3$. One can show that

$$
\begin{aligned}
& H_{1}(\widetilde{\Gamma}, \mathbb{Z})=\mathbb{Z}\left\langle\left(\tilde{e}_{1}^{+}-\tilde{e}_{1}^{-}\right)+\left(\tilde{f}^{+}-\tilde{f}^{-}\right), \ldots,\left(\tilde{e}_{n_{1}}^{+}-\tilde{e}_{n_{1}}^{-}\right)+\left(\tilde{f}^{+}-\tilde{f}^{-}\right),\right. \\
& \tilde{E}_{1}^{+}-\tilde{E}_{1}^{-}, \ldots, \tilde{E}_{n_{2}}^{+}-\tilde{E}_{n_{2}}^{-},\left(\tilde{e}_{1}^{+}-\tilde{e}_{2}^{-}\right)+\left(\tilde{f}^{+}-\tilde{f}^{-}\right), \ldots,\left(\tilde{e}_{n_{1}-1}^{+}-\tilde{e}_{n_{1}}^{-}\right)+\left(\tilde{f}^{+}-\tilde{f}^{-}\right), \\
& \left.\left(\tilde{e}_{n_{1}}^{+}-\tilde{E}_{1}^{-}\right)+\tilde{f}^{+}, \tilde{E}_{1}^{+}-\tilde{E}_{2}^{-}, \ldots, \tilde{E}_{n_{2}-1}^{+}-\tilde{E}_{n_{2}}^{-}\right\rangle
\end{aligned}
$$

and

$$
\begin{array}{r}
H_{1}(\widetilde{\Gamma}, \mathbb{Z})^{[-]}=\mathbb{Z}\left\langle\left(\tilde{e}_{1}^{+}-\tilde{e}_{1}^{-}\right)+\left(\tilde{f}^{+}-\tilde{f}^{-}\right), \frac{1}{2}\left(\tilde{e}_{1}^{+}-\tilde{e}_{1}^{-}\right)+\frac{1}{2}\left(\tilde{e}_{2}^{+}-\tilde{e}_{2}^{-}\right)+\left(\tilde{f}^{+}-\tilde{f}^{-}\right), \ldots,\right. \\
\frac{1}{2}\left(\tilde{e}_{n_{1}-1}^{+}-\tilde{e}_{n_{1}-1}^{-}\right)+\frac{1}{2}\left(\tilde{e}_{n_{1}}^{+}-\tilde{e}_{n_{1}}^{-}\right)+\left(\tilde{f}^{+}-\tilde{f}^{-}\right), \frac{1}{2}\left(\tilde{e}_{n_{1}}^{+}-\tilde{e}_{n_{1}}^{-}\right)+\frac{1}{2}\left(\tilde{E}_{1}^{+}-\tilde{E}_{1}^{-}\right)+\frac{1}{2}\left(\tilde{f}^{+}-\tilde{f}^{-}\right), \\
\left.\frac{1}{2}\left(\tilde{E}_{1}^{+}-\tilde{E}_{1}^{-}\right)+\frac{1}{2}\left(\tilde{E}_{2}^{+}-\tilde{E}_{2}^{-}\right), \ldots, \frac{1}{2}\left(\tilde{E}_{n_{2}-1}^{+}-\tilde{E}_{n_{2}-1}^{-}\right)+\frac{1}{2}\left(\tilde{E}_{n_{2}}^{+}-\tilde{E}_{n_{2}}^{-}\right)\right\rangle .
\end{array}
$$

The monodromy matrix can then be put in the form

$$
F S_{n_{1}+n_{2}}+\delta_{i}\left(\begin{array}{cccccccc}
2 & -1 & -1 & & & & -1 \\
0 & 1 & 0 & & & & 0 \\
& 0 & 1 & 0 & & & \\
& & & \ddots & & & \\
& & & & 1 & & \\
& & & & & 1 & \\
F S_{n} & & & & & & 1 \\
\hline 2 & 0 & -2 & \cdots & \mid-1 & \cdots & -1
\end{array}\right) .
$$

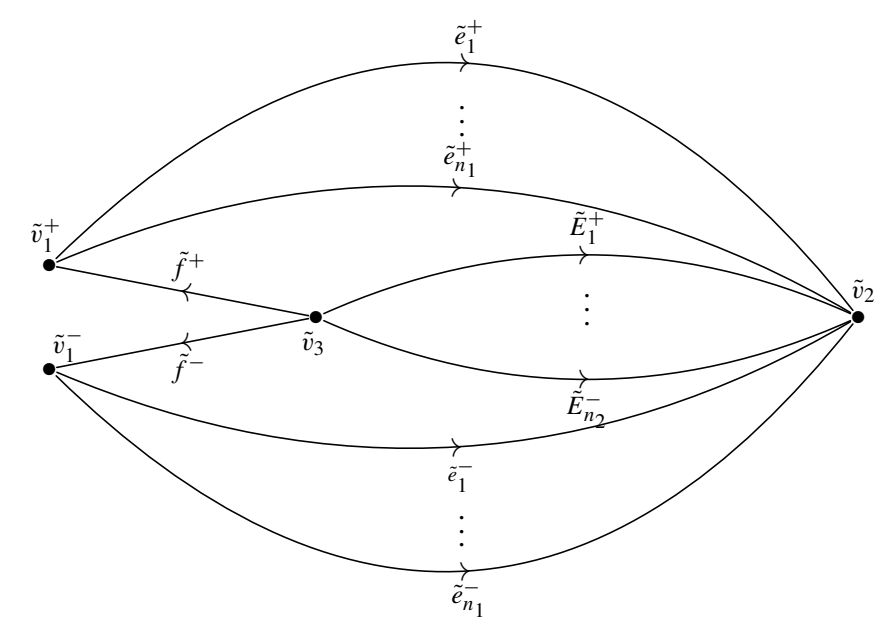

Fig. 16. Dual graph of an $F S_{n_{1}+n_{2}}+\delta_{i}$ degeneration of a Friedman-Smith example with $2 n \geq 2$ nodes. 
The bottom row is a string of the form $2,0,-2,0,-2, \ldots$ of length $n_{1}$ followed by a string of the form $-1,-1,-1, \ldots,-1$ of length $n_{2}$.

Remark C.10. For the $n_{1}=0$ case, we simply have the $F S_{n}$ matrix (the bottom row does not appear). Note also that for the $n_{2}=0$ case, the bottom row is divided by 2 (so that it is primitive).

Lemma C.11. For the $F S_{n_{1}+n_{2}}+\delta_{i}$ examples, if $n=n_{1}+n_{2} \leq 5$, then the monodromy cone is not contained in a cone in the PCD if and only if the example is a degeneration of an $\mathrm{FS}_{2}$ or an $\mathrm{FS}_{3}$ example. Moreover:

(1) If $n_{1}=0,1$, the monodromy cone is an $F S_{n}$ cone, and so it is contained in a cone in the $P C D$ if and only if $n \neq 2,3$.

(2) If $n_{2}=0$, the monodromy cone is the same as for Lemma C.4. Thus if in addition $n \leq 7$, then the monodromy cone is not contained in a cone in the PCD if and only if $n=2,3$.

(3) If $n_{2}=1,2$ or $n_{1}+n_{2}=2,3$, the monodromy cone is not contained in a cone in the $P C D$ (these are exactly the examples that are degenerations of an $F_{2}$ or $F S_{3}$ ).

Proof. The only things to show are the cases $n=4,5$. Suppose that $n=4$. For $n_{1}=0,1$, we can conclude by (1). For $n_{1}=2,3$, we have $n_{2}=2,1$, so we can conclude by (3). If $n_{1}=4$, then $n_{2}=0$, and then we can conclude by (2).

The same analysis works for $n=5$, except when $n_{1}=2$ and $n_{2}=3$ (monodromy matrix below, left). We thank Mathieu Dutour Sikirić for providing us with the metric $Q_{5}^{\prime}$ (below, right), which shows that the cone is contained in (but not equal to) a cone in the PCD.

$$
\left(\begin{array}{ccccc}
2 & -1 & -1 & -1 & -1 \\
0 & 1 & 0 & 0 & 0 \\
0 & 0 & 1 & 0 & 0 \\
0 & 0 & 0 & 1 & 0 \\
0 & 0 & 0 & 0 & 1 \\
\hline 2 & 0 & -1 & -1 & -1
\end{array}\right) \quad Q_{5}^{\prime}=\left(\begin{array}{ccccc}
2 / 3 & 1 / 6 & 1 / 3 & 1 / 3 & 1 / 3 \\
1 / 6 & 2 / 3 & 0 & 0 & 0 \\
1 / 3 & 0 & 2 / 3 & 0 & 0 \\
1 / 3 & 0 & 0 & 2 / 3 & 0 \\
1 / 3 & 0 & 0 & 0 & 2 / 3
\end{array}\right)
$$

\section{C.5. Friedman-Smith-Beauville degenerations $F S_{n}+B_{m}$}

In this section, we consider the case where we replace a vertex of a Friedman-Smith graph in $F S_{n}$ with a Beauville example with $m$ edges (that is, we simply add $m$ fixed loops to the graph at one of the vertices). We will denote these degenerations by $F S_{n}+B_{m}$.

Lemma C.12. For an $F S_{n}+B_{m}$ degeneration, the monodromy cone is the same as for an $\mathrm{FS}_{n}$ cover. Consequently, the monodromy cone is not contained in a cone in the PCD if and only if $n=2,3$.

Proof. See Lemma D.5. 
C.6. Friedman-Smith-Elementary Étale degenerations $F S_{n}+E E_{m}$

In this section, we consider the case where we replace a vertex of a Friedman-Smith graph in $F S_{n}$ with an elementary example with $2 m$ edges (that is, we simply add $2 m$ loops to the graph at one of the vertices, with the loops interchanged pairwise by the involution). We will denote these degenerations by $F S_{n}+E E_{m}$.

Lemma C.13. For an $F S_{n}+E E_{m}$ degeneration, the monodromy matrix can be put in the form

$$
\left(\begin{array}{c|c}
F S_{n} & 0 \\
\hline 0 & \operatorname{Id}_{m}
\end{array}\right)
$$

Consequently, the monodromy cone is not contained in a cone in the PCD if and only if $n=2$, 3. For $n=2,3$, the decomposition into cones in the PCD (as well as SVD and $C C D)$ is given by the star subdivision associated to the face associated to the $F S_{n}$ cone.

Proof. For the monodromy matrix, see Lemma D.3. The statements about the decomposition follow directly from the definitions and the block-diagonal form of the monodromy matrix.

From our computations in these different examples, we are led to the natural question:

Question C.14. For monodromy cones associated to degenerations of Friedman-Smith covers of type $F S_{2}$ or $F S_{3}$, is the second Voronoi decomposition a refinement of the perfect cone decomposition?

\section{Appendix D. Simplifications of monodromy cones}

In this section we discuss some morphisms of homology of dual graphs, which are used in the discussion of Hodge theory. These morphisms also lead to some techniques to simplify the study of the monodromy cones. While we do not strictly need these for the content of this paper, we do include some lemmas below that are similar in spirit, and which we do use to simplify some computations in the preceding sections.

Let $\Gamma$ be a graph, and let $S$ be a subset of the unoriented edges of $\Gamma$. Define $\Gamma \backslash S$ to be the graph obtained by removing the edges in $S$, by which we mean removing both oriented edges lying over an unoriented edge, and $\Gamma / S$ to be the graph obtained by contracting the edges in $S$.

Remark D.1. If $\Gamma$ is the dual graph of a stable curve $X$, and $S$ corresponds to nodes $p_{1}, \ldots, p_{n} \in X$, then $\Gamma \backslash S$ is the dual graph of the curve obtained from $X$ by normalizing at the nodes $p_{1}, \ldots, p_{n}$, and $\Gamma / S$ is the dual graph of the curve obtained from $X$ by smoothing the nodes $p_{1}, \ldots, p_{n}$.

Since $\Gamma \backslash S \subseteq \Gamma$, there is a natural inclusion of complexes

$$
C_{\bullet}(\Gamma \backslash S, \mathbb{Z}) \hookrightarrow C_{\bullet}(\Gamma, \mathbb{Z}) .
$$


There is also a surjective morphism of complexes

$$
C_{\bullet}(\Gamma, \mathbb{Z}) \rightarrow C_{\bullet}(\Gamma / S, \mathbb{Z})
$$

given in the following way. For an edge $e$, we have $e \mapsto 0$ if $e \in S$, and otherwise $e \mapsto e$. The vertices of $\Gamma / S$ correspond to equivalence classes of vertices in $\Gamma$ joined by edges in $S$. The map on vertices is the quotient map. Consequently, if we let $S^{c}$ be the set of unoriented edges of $\Gamma$ complementary to those of $S$, there are natural maps

$$
H_{i}(\Gamma \backslash S, \mathbb{Z}) \rightarrow H_{i}(\Gamma, \mathbb{Z}) \rightarrow H_{i}\left(\Gamma / S^{c}, \mathbb{Z}\right) .
$$

Remark D.2. While the sequence of complexes

$$
C_{\bullet}(\Gamma \backslash S, \mathbb{Z}) \hookrightarrow C_{\bullet}(\Gamma, \mathbb{Z}) \rightarrow C_{\bullet}\left(\Gamma / S^{c}, \mathbb{Z}\right)
$$

is not exact, the following sequence is (split) exact:

$$
0 \rightarrow H_{1}(\Gamma \backslash S, \mathbb{Z}) \rightarrow H_{1}(\Gamma, \mathbb{Z}) \rightarrow H_{1}\left(\Gamma / S^{c}, \mathbb{Z}\right) \rightarrow 0 .
$$

The analogous sequence in degree 0 need not be exact. From the exact sequence on $H_{1}$, in many instances one may choose bases to obtain block-triangular matrices in monodromy computations for Pryms. As an application, one can give a short combinatorial proof that the monodromy cone of a degeneration of a Friedman-Smith cover with at least four nodes $\left(F S_{n}, n \geq 2\right)$ is not matroidal. This provides an alternative combinatorial proof of [Vol02, Prop. 2.1 and p. 120].

The observations in the remark lead to some general techniques for simplifying monodromy cones for Prym varieties. For brevity, we omit these. Two lemmas with similar statements and proofs, which we do use in earlier arguments, are stated below.

Lemma D.3. Let $\widetilde{\Gamma}$ be a graph with admissible involution $\iota$. Suppose that $\widetilde{\Gamma}_{1}, \widetilde{\Gamma}_{2} \subseteq \widetilde{\Gamma}$ are connected subgraphs preserved by the involution $\iota \widetilde{\Gamma}_{i}=\widetilde{\Gamma}_{i}(i=1,2)$. If $\widetilde{\Gamma}=\widetilde{\Gamma}_{1} \cup \widetilde{\Gamma}_{2}$ and $\widetilde{\Gamma}_{1} \cap \widetilde{\Gamma}_{2}=\{v\} \subseteq V(\widetilde{\Gamma})$ is a single vertex of the graph, then the matrix defining the monodromy cone can be put in the form

$$
\operatorname{MC}(\widetilde{\Gamma})=\left(\begin{array}{c|c}
M C\left(\widetilde{\Gamma}_{1}\right) & 0 \\
\hline 0 & M C\left(\widetilde{\Gamma}_{2}\right)
\end{array}\right) .
$$

Proof. The proof is left to the reader.

Remark D.4. In particular, fix an admissible collection of admissible cone decompositions $\Sigma$. Then in the notation of the lemma, $\operatorname{MC}(\widetilde{\Gamma})$ is contained in a cone in $\Sigma$ if and only if $M C\left(\widetilde{\Gamma}_{1}\right)$ and $M C\left(\widetilde{\Gamma}_{2}\right)$ are contained in cones in $\Sigma$.

Lemma D.5. Let $\widetilde{\Gamma}$ be a graph with admissible involution ı. Suppose that $\widetilde{\Gamma}^{\prime}$ is a graph with admissible involution $\iota^{\prime}$, which is obtained from $\widetilde{\Gamma}$ by adding a single loop (an edge $\tilde{e}^{\prime}$ such that $\left.s\left(\tilde{e}^{\prime}\right)=t\left(\tilde{e}^{\prime}\right)\right)$. Then $\operatorname{MC}(\widetilde{\Gamma})=\operatorname{MC}\left(\widetilde{\Gamma}^{\prime}\right)$.

Proof. Necessarily $\iota^{\prime}\left(\tilde{e}^{\prime}\right)=\tilde{e}^{\prime}$. Then apply the previous lemma with $\widetilde{\Gamma}_{1}=\widetilde{\Gamma}$ and $\widetilde{\Gamma}_{2}=$ $\left(s\left(\tilde{e}^{\prime}\right), \tilde{e}^{\prime}\right)$. 


\section{Appendix E (by Mathieu Dutour Sikirić). Extension to the central cone decomposi- tion}

In this appendix we discuss the extension of the Prym map to the central cone compactification. We shall see that the indeterminacy loci differ substantially for the three toroidal compactifications $\bar{A}_{g}^{V}, \bar{A}_{g}^{P}$ and $\bar{A}_{g}^{C}$.

Theorem E.1. For the extension of the Prym map $P_{g}^{C}: \bar{R}_{g+1}-\rightarrow \bar{A}_{g}^{C}$ to the central cone compactification the following holds:

(1) $\overline{F S}_{2} \cup \overline{F S}_{3} \subseteq \operatorname{Ind}\left(P_{g}^{C}\right)$, and for $n \geq 4$ the strata $F S_{n}$ are not contained in $\operatorname{Ind}\left(P_{g}^{C}\right)$.

(2) If $g \geq 9$, the indeterminacy locus $\operatorname{Ind}\left(P_{g}^{C}\right)$ contains points that are not contained in $\bigcup_{n \geq 1} \overline{F S}_{n}$.

Proof. We shall first prove (1). In genus 2 and 3 the central cone decomposition coincides with the second Voronoi and the perfect cone decomposition. In particular the monodromy cones of $F S_{2}$ and $F S_{3}$ are not contained in a cone in the central cone decomposition (CCD). This shows the inclusion $\overline{F S}_{2} \cup \overline{F S}_{3} \subseteq \operatorname{Ind}\left(P_{g}^{C}\right)$. To complete the proof of (1) it remains to show that the monodromy cones of $F S_{n}$ are contained in a cone of the $\mathrm{CCD}$ for $n \geq 4$. For this we work with the representation of $F S_{n}$ given by (A.1), namely

$$
F S_{n} \quad\left(\begin{array}{ccccccc}
2 & -1 & -1 & & & & -1 \\
0 & 1 & 0 & & & & 0 \\
0 & 0 & 1 & 0 & & & \\
& & & \ddots & \ddots & & \\
& & & 0 & 1 & 0 & 0 \\
& & & & 0 & 1 & 0 \\
& & & & & 0 & 1
\end{array}\right) .
$$

We will apply the criterion of Lemma 1.7 using the quadratic form

$$
Q_{C}=\left(\begin{array}{cccccccc}
1 & 1 / 2 & 1 / 2 & 1 / 2 & 0 & \ldots & \ldots & 0 \\
1 / 2 & 1 & 0 & 0 & 0 & \ldots & \ldots & 0 \\
1 / 2 & 0 & 1 & 0 & 0 & \ldots & \ldots & 0 \\
1 / 2 & 0 & 0 & 1 & -1 / 2 & 0 & \ldots & 0 \\
0 & 0 & 0 & -1 / 2 & 1 & -1 / 2 & \ddots & 0 \\
\vdots & \vdots & \vdots & 0 & -1 / 2 & 1 & \ddots & 0 \\
\vdots & \ddots & \ddots & \vdots & \ddots & \ddots & \ddots & -1 / 2 \\
0 & \ldots & \ldots & \ldots & \ldots & 0 & -1 / 2 & 1
\end{array}\right)
$$

Clearly this matrix is integer valued, and one computes immediately that for all rows $\ell_{i}$ of the $F S_{n}$ matrix one has $Q_{C}\left(\ell_{i}\right)=1$. To prove the claim it remains to show that $Q_{C}$ is positive definite. To see this we first note that $Q_{C}$ is equivalent to 


$$
Q_{C}^{\prime}=\left(\begin{array}{cccccccc}
1 & -1 / 2 & -1 / 2 & -1 / 2 & 0 & \ldots & \ldots & 0 \\
-1 / 2 & 1 & 0 & 0 & 0 & \ldots & \ldots & 0 \\
-1 / 2 & 0 & 1 & 0 & 0 & \ldots & \ldots & 0 \\
-1 / 2 & 0 & 0 & 1 & -1 / 2 & 0 & \ldots & 0 \\
0 & 0 & 0 & -1 / 2 & 1 & -1 / 2 & \ddots & 0 \\
\vdots & \vdots & \vdots & 0 & -1 / 2 & 1 & \ddots & 0 \\
\vdots & \ddots & \ddots & \vdots & \ddots & \ddots & \ddots & -1 / 2 \\
0 & \ldots & \ldots & \ldots & \ldots & 0 & -1 / 2 & 1
\end{array}\right) .
$$

But then taking $e_{1}, \ldots, e_{n}$ as a $\mathbb{Z}$-basis of $\mathbb{Z}^{n}$, we have $Q_{C}^{\prime}\left(e_{i}\right)=1, Q_{C}^{\prime}\left(e_{1}, e_{i}\right)=-1 / 2$ for $i=2,3,4$ and $Q_{C}^{\prime}\left(e_{i}, e_{i+1}\right)=-1 / 2, i=4, \ldots, n-1$, with $Q_{C}^{\prime}\left(e_{k}, e_{l}\right)=0$ in all other cases. This shows that $Q_{C}^{\prime}$ is of the form $D_{n}$ (up to scalar), proving positive definiteness.

We shall now prove (2). The starting point is that by $\left[\mathrm{AB} 12, \mathrm{AL}^{+} 12\right]$ there are stable curves of genus $g \geq 9$ near which the Torelli map to $\bar{A}_{g}^{C}$ is not a morphism. We start with two copies of a curve $C$ of genus $g$ with two marked points, say $P, Q$. Then we attach $P$ to $Q$ and vice versa. On the resulting curve we can define an involution that swaps the two components and the two nodes. The associated Prym variety is then equal to the Jacobian of $C$. We can now degenerate this involution to an example which proves our claim. Indeed, since $g \geq 9$ we can degenerate $C$ to a curve $C^{\prime}$ which lies in the indeterminacy locus of the Torelli map to $\bar{A}_{g}^{C}$. We now choose one component of $C^{\prime}$, and attach it to a second copy of $C^{\prime}$ as in the Wirtinger example with 2 nodes (to the same chosen component on the second copy of $C^{\prime}$ ). Again we let the involution swap the two copies of $C^{\prime}$ (and the chosen attaching nodes). The monodromy cone is then the same as the monodromy cone associated to $C^{\prime}$ (cf. the Wirtinger Example B.3). This shows that the map $P_{g}^{C}: \bar{R}_{g+1} \rightarrow \bar{A}_{g}^{C}$ is not a morphism near this cover. It remains to check that this is not contained in $\bigcup_{n \geq 1} \overline{F S}_{n}$. But this follows from considering the dual graph: since the vertices of this graph are interchanged pairwise, it cannot be contracted to the graph (with involution) of an $F S_{n}$ example.

Acknowledgments. We are grateful to Mathieu Dutour Sikirić who was always willing to answer our questions on cone decompositions and who helped us check some of our guesses with his powerful computer programs.

Research of the first author was supported in part by NSF grant DMS-11-01333 and Simons Foundation Collaboration Grant for Mathematicians (317572). Research of the second author is supported in part by NSF grant DMS-12-01369. Research of the third author is supported in part by DFG grant Hu-337/6-2. Research of the fourth author is supported in part by NSF grants DMS12-00875, DMS-12-54812, and by a Sloan Fellowship.

\section{References}

[Ale02] Alexeev, V.: Complete moduli in the presence of semiabelian group action. Ann. of Math. (2) 155, 611-708 (2002) Zbl 1052.14017 MR 1923963

[ABH02] Alexeev, V., Birkenhake, Ch., Hulek, K.: Degenerations of Prym varieties. J. Reine Angew. Math. 553, 73-116 (2002) Zbl 1008.14004 MR 1944808 
[AB12] Alexeev, V., Brunyate, A.: Extending the Torelli map to toroidal compactifications of Siegel space. Invent. Math. 188, 175-196 (2012) Zbl 1242.14042 MR 2897696

[AL $\left.{ }^{+} 12\right]$ Alexeev, V., Livingston, R., Tenini, J., Arap, M., Hu, X., Huckaba, L., McFaddin, P., Musgrave, S., Shin, J., Ulrich, C.: Extended Torelli map to the Igusa blowup in genus 6, 7, and 8. Experimental Math. 21, 193-203 (2012) Zbl 1246.14037 MR 2931314

$\left[\mathrm{AM}^{+} 10\right]$ Ash, A., Mumford, D., Rapoport, M., Tai, Y.-S.: Smooth Compactifications of Locally Symmetric Varieties. 2nd ed., Cambridge Univ. Press, Cambridge (2010) Zbl 1209.14001 MR 2590897

[Bea77] Beauville, A.: Prym varieties and the Schottky problem. Invent. Math. 41, 149-196 (1977) Zbl 0333.14013 MR 0572974

[Ber99] Bernstein, M.: Moduli of curves with level structure. Ph.D. Thesis, Harvard Univ. (1999) MR 2699319

[Bor72] Borel, A.: Some metric properties of arithmetic quotients of symmetric spaces and an extension theorem. J. Differential Geom. 6, 543-560 (1972) Zbl 0249.32018 MR 0338456

[CML09] Casalaina-Martin, S., Laza, R.: The moduli space of cubic threefolds via degenerations of the intermediate Jacobian. J. Reine Angew. Math. 633, 29-65 (2009) Zbl 1248.14041 MR 2561195

[CML13] Casalaina-Martin, S., Laza, R.: Simultaneous semi-stable reduction for curves with ADE singularities. Trans. Amer. Math. Soc. 365, 2271-2295 (2013) Zbl 1300.14031 MR 3020098

[Cat84] Cattani, E. H.: Mixed Hodge structures, compactifications and monodromy weight filtration. In: Topics in Transcendental Algebraic Geometry (Princeton, NJ, 1981/1982), Ann. of Math. Stud. 106, Princeton Univ. Press, Princeton, NJ, 75-100 (1984) Zbl 0579.14010 MR 0756847

[Del74] Deligne, P.: Théorie de Hodge. III. Inst. Hautes Études Sci. Publ. Math. 44, 5-77 (1974) Zbl 0237.14003 MR 0498552

[Don92] Donagi, R.: The fibers of the Prym map. In: Curves, Jacobians, and Abelian Varieties (Amherst, MA, 1990), Contemp. Math. 136, Amer. Math. Soc., Providence, RI, 55-125 (1992) Zbl 0783.14025 MR 1188194

[DHS13] Dutour Sikirić, M., Hulek, K., Schürmann, A.: Smoothness and singularities of the perfect form and the second Voronoi compactification of $\mathcal{A}_{g}$. Algebr. Geom. 2, 642-653 (2015) Zbl 1337.14036

[ER01] Erdahl, R., Rybnikov, K.: Voronoi-Dickson hypothesis on perfect forms and L-types. arXiV:math/0112097, 21 pp. (2001)

[ER94] Erdahl, R. M., Ryshkov, S. S.: On lattice dicing. Eur. J. Combin. 15, 459-481 (1994) Zbl 0809.52019 MR 1292957

[FC90] Faltings, G., Chai, C.-L.: Degeneration of Abelian Varieties. Ergeb. Math. Grenzgeb. (3) 22, Springer, Berlin (1990) Zbl 0744.14031 MR 1083353

[Far12] Farkas, G.: Prym varieties and their moduli. In: Contributions to Algebraic Geometry, EMS Ser. Congr. Rep., Eur. Math. Soc., Zürich, 215-255 (2012) Zbl 1259.14032 MR 2976944

[FL10] Farkas, G., Ludwig, K.: The Kodaira dimension of the moduli space of Prym varieties. J. Eur. Math. Soc. 12, 755-795 (2010) Zbl 1193.14043 MR 2639318

[FS86] Friedman, R., Smith, R. C.: Degenerations of Prym varieties and intersections of three quadrics. Invent. Math. 85, 615-635 (1986) Zbl 0619.14027 MR 0848686

[GH11] Grushevsky, S., Hulek, K.: Principally polarized semi-abelic varieties of small torus rank, and the Andreotti-Mayer loci. Pure Appl. Math. Quart. 7, 1309-1360 (2011) Zbl 1251.14030 MR 2918163 
[GH12] Grushevsky, S., Hulek, K.: The class of the locus of intermediate Jacobians of cubic threefolds. Invent. Math. 190, 119-168 (2012) Zbl 1272.14032 MR 2969275

[GHT15] Grushevsky, S., Hulek, K., Tommasi, O.: Stable cohomology of the perfect cone toroidal compactification of the moduli space of abelian varieties. J. Reine Angew. Math. (online) (2015), DOI: 10.1515/crelle-2015-0067

[MV12] Melo, M., Viviani, F.: Comparing perfect and 2nd Voronoi decompositions: the matroidal locus. Math. Ann. 354, 1521-1554 (2012) Zbl 1264.11050 MR 2993003

[Mor84] Morrison, D. R.: The Clemens-Schmid exact sequence and applications. In: Topics in Transcendental Algebraic Geometry (Princeton, NJ, 1981/1982), Ann. of Math. Stud. 106, Princeton Univ. Press, Princeton, NJ, 101-119 (1984) Zbl 0576.32034 MR 0756848

[Nam76] Namikawa, Y.: A new compactification of the Siegel space and degeneration of Abelian varieties. II. Math. Ann. 221, 201-241 (1976) Zbl 0327.14013 MR 0480538

[Nam80] Namikawa, Y.: Toroidal Compactification of Siegel Spaces. Lecture Notes in Math. 812, Springer, Berlin (1980) Zbl 0466.14011 MR 0584625

[Ols08] Olsson, M.: Compactifying Moduli Spaces for Abelian Varieties. Lecture Notes in Math. 1958, Springer, Berlin (2008) Zbl 1165.14004 MR 2446415

[RB78] Ryshkov, S. S., Baranovski1̆, E. P.: $C$-types of $n$-dimensional lattices and 5-dimensional primitive parallelohedra (with application to the theory of coverings). Proc. Steklov Inst. Math. 1978, no. 4, 140 pp.; translation of Trudy Mat. Inst. Steklov. 137 (1976) Zbl 0419.10031 MR 0535314

[RE88] Ryshkov, S. S., Erdahl, R. M.: The empty sphere. II. Canad. J. Math. 40, 1058-1073 (1988) Zbl 0653.10027 MR 0973509

[Sch73] Schmid, W.: Variation of Hodge structure: the singularities of the period mapping. Invent. Math. 22, 211-319 (1973) Zbl 0278.14003 MR 0382272

[Ste76] Steenbrink, J.: Limits of Hodge structures. Invent. Math. 31, 229-257 (1975/76) Zbl 0303.14002 MR 0429885

[Vol02] Vologodsky, V.: The locus of indeterminacy of the Prym map. J. Reine Angew. Math. 553, 117-124 (2002) Zbl 1008.14005 MR 1944809

[Vol04] Vologodsky, V.: On fibers of the toric resolution of the extended Prym map. Proc. Amer. Math. Soc. 132, 3159-3165 (2004) Zbl 1073.14044 MR 2073289 